JOURNAL OF THE

AMERICAN MATHEMATICAL SOCIETY

Volume 15, Number 2, Pages 295-366

S 0894-0347(02)00387-9

Article electronically published on January 18, 2002

\title{
NOETHERIAN HEREDITARY ABELIAN CATEGORIES SATISFYING SERRE DUALITY
}

\author{
I. REITEN AND M. VAN DEN BERGH
}

\section{Contents}

Notations and conventions $\quad 296$

Introduction $\quad 296$

I. Serre duality and almost split sequences $\quad 300$

I.1. Preliminaries on Serre duality 300

I.2. Connection between Serre duality and Auslander-Reiten triangles 304

I.3. Serre functors on hereditary abelian categories 307

II. Hereditary noetherian abelian categories with non-zero projective objects 309

II.1. Hereditary abelian categories constructed from quivers 310

II.2. Hereditary abelian categories generated by preprojectives 314

\begin{tabular}{ll|l|}
\hline II.3. Derived equivalences & 318 \\
\hline
\end{tabular}

II.4. The classification 324

III. Sources of hereditary abelian categories with no projectives or injectives 329

III.1. Hereditary abelian categories with Serre functor and all objects of finite length 329

III.2. Sheaves of hereditary orders and graded rings 331

III.3. Hereditary abelian categories associated to infinite Dynkin and tame quivers

IV. Hereditary noetherian abelian categories with no projectives or injectives

IV.1. Preliminaries

IV.2. Completion 349

IV.3. Description in terms of a pullback diagram 353

\begin{tabular}{ll|l|}
\hline IV.4. The finite orbit case & 356 \\
\hline
\end{tabular}

IV.5. The infinite orbit case 358

V. Applications $\quad 360$

V.1. Saturatedness 361

Received by the editors December 6, 2000.

2000 Mathematics Subject Classification. Primary 18E10, 18G20, 16G10, 16G20, 16G30, $16 \mathrm{G} 70$.

Key words and phrases. Noetherian hereditary abelian categories, Serre duality, saturation property.

The second author is a senior researcher at the Fund for Scientific Research. The second author also wishes to thank the Clay Mathematics Institute for material support during the period in which this paper was written. 
V.2. Graded rings $\quad 362$

Appendix A. Some results on abelian categories $\quad 363$

\begin{tabular}{ll|} 
References & 365 \\
\hline
\end{tabular}

\section{NOTATIONS AND CONVENTIONS}

Most notations will be introduced locally. The few global ones are given below. Unless otherwise specified $k$ will be an algebraically closed field and all rings and categories in this paper will be $k$-linear.

If $A$ is a $\operatorname{ring}$, then $\bmod (A)$ will be the category of finitely generated right $A$ modules. Similarly if $R$ is a $\mathbb{Z}$-graded ring, then $\operatorname{gr}(R)$ will be the category of finitely generated graded right modules with degree zero morphisms, and $\operatorname{Gr}(R)$ the category of all graded right $R$-modules. If $R$ is noetherian, then following [3] $\operatorname{tors}(R)$ will be the full subcategory of $\operatorname{gr}(R)$ consisting of graded modules with right bounded grading. Also following [3] we put $\operatorname{qgr}(R)=\operatorname{gr}(R) / \operatorname{tors}(R)$. $\mathcal{C}$.

For an abelian category $\mathcal{C}$ we denote by $D^{b}(\mathcal{C})$ the bounded derived category of

\section{INTRODUCTION}

One of the goals of non-commutative algebraic geometry is to obtain an understanding of $k$-linear abelian categories $\mathcal{C}$, for a field $k$, which have properties close to those of the category of coherent sheaves over a non-singular proper scheme. Hence some obvious properties one may impose on $\mathcal{C}$ in this context are the following:

- $\mathcal{C}$ is Ext-finite, i.e.

$$
\operatorname{dim}_{k} \operatorname{Ext}^{i}(A, B)<\infty
$$

for all $A, B \in \mathcal{C}$ and for all $i$.

- $\mathcal{C}$ has homological dimension $n<\infty$, i.e.

$$
\operatorname{Ext}^{i}(A, B)=0
$$

for $A, B \in \mathcal{C}$ and $i>n$, and $n$ is minimal with this property.

Throughout this paper $k$ will denote a field, and even though it is not always necessary we will for simplicity assume that $k$ is algebraically closed. All categories will be $k$-linear. When we say that $\mathcal{C}$ is Ext-finite, it will be understood that this is with respect to the field $k$.

In most of this paper we will assume that $\mathcal{C}$ is an Ext-finite abelian category of homological dimension at most 1 , in which case we say that $\mathcal{C}$ is hereditary.

A slightly more subtle property of non-singular proper schemes is Serre duality. Let $X$ be a non-singular proper scheme over $k$ of dimension $n$, and let $\operatorname{coh}(X)$ denote the category of coherent $\mathcal{O}_{X}$-modules. Then the classical Serre duality theorem asserts that for $\mathcal{F} \in \operatorname{coh}(X)$ there are natural isomorphisms

$$
H^{i}(X, \mathcal{F}) \cong \operatorname{Ext}^{n-i}\left(\mathcal{F}, \omega_{X}\right)^{*}
$$

where $(-)^{*}=\operatorname{Hom}_{k}(-, k)$.

A very elegant reformulation of Serre duality was given by Bondal and Kapranov in [8]. It says that for any $\mathcal{E}, \mathcal{F} \in D^{b}(\operatorname{coh}(X))$ there exist natural isomorphisms

$$
\operatorname{Hom}_{D^{b}(\operatorname{coh}(X))}(\mathcal{E}, \mathcal{F}) \cong \operatorname{Hom}_{D^{b}(\operatorname{coh}(X))}\left(\mathcal{F}, \mathcal{E} \otimes \omega_{X}[n]\right)^{*} .
$$


Stated in this way the concept of Serre duality can be generalized to certain abelian categories.

- $\mathcal{C}$ satisfies Serre duality if it has a so-called Serre functor. The latter is by definition an autoequivalence $F: D^{b}(\mathcal{C}) \rightarrow D^{b}(\mathcal{C})$ such that there are isomorphisms

$$
\operatorname{Hom}(A, B) \cong \operatorname{Hom}(B, F A)^{*}
$$

which are natural in $A, B$.

On the other hand hereditary abelian Ext-finite categories $\mathcal{C}$ with the additional property of having a tilting object have been important for the representation theory of finite dimensional algebras. Recall that $T$ is a tilting object in $\mathcal{C}$ if $\operatorname{Ext}^{1}(T, T)=0$, and if $\operatorname{Hom}(T, X)=0=\operatorname{Ext}^{1}(T, X)$ implies that $X$ is 0 . These categories $\mathcal{C}$ are important in the study of quasitilted algebras, which by definition are the algebras of the form $\operatorname{End}_{\mathcal{C}}(T)$ for a tilting object $T$ [16], and which contain the important classes of tilted and canonical algebras. A prominent property in the representation theory of finite dimensional algebras is having almost split sequences, and also the Ext-finite hereditary abelian categories with tilting object have this property [16].

In view of the above it is interesting, and useful, to investigate the relationship between Serre duality and almost split sequences. In fact, this relationship is very close in the hereditary case. The more general connections are on the level of triangulated categories, replacing almost split sequences with Auslander-Reiten

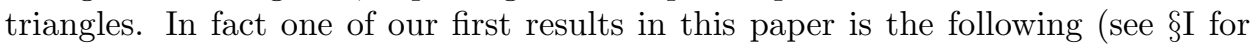
more complete results):

Theorem A. 1. $\mathcal{C}$ has a Serre functor if and only if $D^{b}(\mathcal{C})$ has AuslanderReiten triangles (as defined in [14]).

2. If $\mathcal{C}$ is hereditary, then $\mathcal{C}$ has a Serre functor if and only if $\mathcal{C}$ has almost split sequences and there is a one-one correspondence between the indecomposable projective objects $P$ and the indecomposable injective objects $I$, such that the simple top of $P$ is isomorphic to the socle of $I$.

Hence Ext-finite hereditary abelian categories with Serre duality are of interest both for non-commutative algebraic geometry and for the representation theory of finite dimensional algebras. The main result of this paper is the classification of the noetherian ones.

To be able to state our result we first give a list of hereditary abelian categories satisfying Serre duality.

(a) If $\mathcal{C}$ consists of the finite dimensional nilpotent representations of the quiver $\tilde{A}_{n}$ or of the quiver $A_{\infty}^{\infty}$, with all arrows oriented in the same direction, then it is classical that $\mathcal{C}$ has almost split sequences, and hence Serre duality.

(b) Let $X$ be a non-singular projective connected curve over $k$ with function field $K$, and let $\mathcal{O}$ be a sheaf of hereditary $\mathcal{O}_{X}$-orders in $M_{n}(K)$ (see [21]). Then one proves exactly as in the commutative case that $\operatorname{coh}(\mathcal{O})$ satisfies Serre duality.

(c) Let $Q$ be either $A_{\infty}^{\infty}$ or $D_{\infty}$ with zig-zag orientation. It is shown in $\lcm{1 I I .3}$ that there exists a noetherian hereditary abelian category $\mathcal{C}$ which is derived equivalent to the category $\operatorname{rep}(Q)$ of finitely presented representations of $Q$, and which has no non-zero projectives or injectives. Depending on $Q$ we call this category the $\mathbb{Z} A_{\infty}^{\infty}$ or the $\mathbb{Z} D_{\infty}$ category. Since Serre duality is defined in terms of the derived category, it follows that $\mathcal{C}$ satisfies Serre duality. 
If $Q=A_{\infty}^{\infty}$, then $\mathcal{C}$ is nothing but the category $\operatorname{gr}_{\mathbb{Z}^{2}}(k[x, y]) /($ finite length) considered in [30]. If $Q=D_{\infty}$ and chark $\neq 2$, then $\mathcal{C}$ is a skew version of the $\mathbb{Z} A_{\infty}^{\infty}$ category (see $\left.\oint \amalg I I .3\right)$. The $\mathbb{Z} A_{\infty}^{\infty}$ category and the $\mathbb{Z} D_{\infty}$ category have also been considered by Lenzing.

(d) We now come to more subtle examples (see $₫$ III). Let $Q$ be a connected quiver. Then for a vertex $x \in Q$ we have a corresponding projective representation $P_{x}$ and an injective representation $I_{x}$. If $Q$ is locally finite and there is no infinite path ending at any vertex, the functor $P_{x} \mapsto I_{x}$ may be derived to yield a fully faithful endofunctor $F: D^{b}(\operatorname{rep}(Q)) \rightarrow D^{b}(\operatorname{rep}(Q))$. Then $F$ behaves like a Serre functor, except that it is not in general an autoequivalence. We call such $F$ a right Serre functor (see 1 .1.1). Luckily given a right Serre functor there is a formal procedure to invert it so as to obtain a true Serre functor (Theorem [I.1.3). This yields a hereditary abelian category $\widetilde{\text { rep }}(Q)$ which satisfies Serre duality. Under the additional hypotheses that $Q$ consists of a subquiver $Q_{o}$ with no path of infinite length, with rays attached to vertices of $Q_{o}$, then $\widetilde{\text { rep }}(Q)$ turns out to be noetherian (see Theorem 11.4 .3 ). Here we mean by a ray an $A_{\infty}$ quiver with no vertex which is a sink. An interesting feature of the noetherian categories $\widetilde{\text { rep }}(Q)$, exhibiting a new type of behavior, is that they are generated by the preprojective objects, but not necessarily by the projective objects.

Now we can state our main result. Recall that an abelian category $\mathcal{C}$ is connected if it cannot be non-trivially written as a direct $\operatorname{sum} \mathcal{C}_{1} \oplus \mathcal{C}_{2}$.

Theorem B. Let $\mathcal{C}$ be a connected noetherian Ext-finite hereditary abelian category satisfying Serre duality. Then $\mathcal{C}$ is one of the categories described in (a)-(d) above.

The cases (a), (b), (c) are those where there are no non-zero projective objects. Those in (a) are exactly the $\mathcal{C}$ where all objects have finite length. For the $\mathcal{C}$ having some objects of infinite length, then either all indecomposable objects of finite length have finite $\tau$-period (case (b)) or all have infinite $\tau$-period (case (c)). Here the object $\tau C$ is defined by the almost split sequence $0 \rightarrow \tau C \rightarrow B \rightarrow C \rightarrow 0$ for $C$ indecomposable in $\mathcal{C}$, and we have $\tau C=F(C)[-1]$.

Under the additional assumption that $\mathcal{C}$ has a tilting object, such a classification was given in 19. The only cases are the categories of finitely generated modules $\bmod A$ for a finite dimensional indecomposable hereditary $k$-algebra $A$ and the categories coh $\mathbb{X}$ of coherent sheaves on a weighted projective line $\mathbb{X}$ in the sense of 12]. From the point of view of the above list of examples the first case corresponds to the finite quivers $Q$ in (d), in which case $\widetilde{\operatorname{rep}}(Q)=\operatorname{rep}(Q)$ is equivalent to $\bmod (k Q)$, where $k Q$ is the path algebra of $Q$ over $k$. The categories coh $\mathbb{X}$ are a special case of (b), corresponding to the case where the projective curve is $\mathbb{P}^{1}$ (see [23]).

Our proof of Theorem B is rather involved and covers the first four sections. The main steps are as follows:

1. In the first two subsections of $\oint[$ we construct the categories $\widetilde{\operatorname{rep}}(Q)$, and we show that they are characterized by the property of having noetherian injectives and being generated by preprojectives.

2. In $₫ I I .4$ we give necessary and sufficient conditions for $\widetilde{\text { rep }}(Q)$ to be noetherian, and furthermore we prove a decomposition theorem which states that an Ext-finite noetherian hereditary abelian category with Serre functor can be 
decomposed as a direct sum of a hereditary abelian category which is generated by preprojectives and a hereditary abelian category which doesn't have non-zero projectives or injectives.

3 . We are now reduced to the case where there are no non-zero projectives or injectives. The case where all objects have finite length is treated in III.1.

4. The case where there are no non-zero projectives or injectives and at least one object of infinite length is covered in $₫[\mathrm{IV}$ It turns out that this case naturally falls into two subcases:

$(\alpha)$ The simple objects are $\tau$-periodic. In that case, using the results in [3], we show that $\mathcal{C}$ is of the form $\operatorname{qgr}(R)$ for $R$ a two-dimensional commutative graded ring, where $\operatorname{qgr}(R)$ is the quotient category gr $R$ /finite length. Using 11 it then follows that $\mathcal{C}$ is of the form (b).

( $\beta$ ) The simple objects are not $\tau$-periodic. We show that if such $\mathcal{C}$ exists, then it is characterized by the fact that it has either one or two $\tau$-orbits of simple objects. Since the $\mathbb{Z} A_{\infty}^{\infty}$ and $\mathbb{Z} D_{\infty}$ category have this property, we are done.

Our methods for constructing the new hereditary abelian categories $\widetilde{\operatorname{rep}}(Q)$ are somewhat indirect although we believe they are interesting. After learning about our results Claus Ringel has recently found a more direct construction for these categories [24.

All the hypotheses for Theorem B are necessary. For example the non-commutative curves considered in [30] are noetherian hereditary abelian categories of Krulldimension one which in general do not satisfy Serre duality (except for the special case listed in (c)). If $\mathcal{C}$ is the opposite category to one of the categories (b), (c), (d), then it is not noetherian, but it satisfies the other hypotheses.

Nevertheless it is tempting to ask whether a result similar to Theorem B remains valid without the noetherian hypothesis if we work up to derived equivalence. In particular, is any such category derived equivalent to a noetherian one? Under the additional assumption that $\mathcal{C}$ has a tilting object, this has been proved by Happel in [15], and it has recently been shown by Ringel that this is not true in general [25].

In the final section we use Theorem B to draw some conclusions on the structure of certain hereditary abelian categories.

To start with we discuss the "saturation" property. This is a subtle property of certain abelian categories which was discovered by Bondal and Kapranov [8]. Recall that a cohomological functor $H: D^{b}(\mathcal{C}) \rightarrow \bmod (k)$ is of finite type if for every $A \in D^{b}(\mathcal{C})$ only a finite number of $H(A[n])$ are non-zero. We have already defined what it means for $\mathcal{C}$ to have homological dimension $n<\infty$. It will be convenient to say more generally that $\mathcal{C}$ has finite homological dimension if for any $A, B$ in $\mathcal{C}$ there is at most a finite number of $i$ with $\operatorname{Ext}^{i}(A, B) \neq 0$. In particular, the analogue of this definition makes sense for triangulated categories.

- Let $\mathcal{C}$ be an Ext-finite abelian category of finite homological dimension. Then $\mathcal{C}$ is saturated if every cohomological functor $H: D^{b}(\mathcal{C}) \rightarrow \bmod (k)$ of finite type is of the form $\operatorname{Hom}(A,-)$ (i.e. $H$ is representable).

It is easy to show that a saturated category satisfies Serre duality. It was shown in 8 ] that $\operatorname{coh}(X)$ for $X$ a non-singular projective scheme is saturated and that saturation also holds for categories of the form $\bmod (\Lambda)$ with $\Lambda$ a finite dimensional algebra. Inspired by these results we prove the following result in $\S \mathrm{V} .1$ 
Theorem C. Assume that $\mathcal{C}$ is a saturated connected noetherian Ext-finite hereditary abelian category. Then $\mathcal{C}$ has one of the following forms:

1. $\bmod (\Lambda)$ where $\Lambda$ is a connected finite dimensional hereditary $k$-algebra.

2. $\operatorname{coh}(\mathcal{O})$ where $\mathcal{O}$ is a sheaf of hereditary $\mathcal{O}_{X}$-orders (see (b) above) over a non-singular connected projective curve $X$.

It is easy to see that the hereditary abelian categories listed in the above theorem are of the form $\operatorname{qgr}(R)$. We refer the reader to 9 (see also $\$ \nabla .2$ ) where it is shown in reasonable generality that abelian categories of the form $\operatorname{qgr}(R)$ are saturated.

There are also applications to the relationship between existence of tilting objects and the Grothendieck group being finitely generated. This was one of the original motivations for this work, and is dealt with in another paper [23].

We would like to thank Claus Ringel for helpful comments on the presentation of this paper.

\section{Serre Duality and Almost SPlit Sequences}

It has been known for some time that there is a connection between classical Serre duality and existence of almost split sequences. There is a strong analogy between the Serre duality formula for curves and the formula $D\left(\operatorname{Ext}_{\Lambda}^{1}(C, A)\right) \simeq$ $\overline{\operatorname{Hom}}(A, \mathrm{DTr} C)$ for artin algebras (where $D=\operatorname{Hom}_{k}(-, k)$ ), on which the existence of almost split sequences is based (see [5]). Actually, existence of almost split sequences in some sheaf categories for curves can be proved either by using an analogous formula for graded maximal Cohen-Macaulay modules or by using Serre duality [4, 27]. The notion of almost split sequences was extended to the notion of Auslander-Reiten triangles in triangulated categories [14, and existence of such was proved for $D^{b}(\bmod \Lambda)$ when $\Lambda$ is a $k$-algebra of finite global dimension [14. In this case the corresponding translate is given by an equivalence of categories. On the other hand an elegant formulation of Serre duality in the bounded derived category, together with a corresponding Serre functor, was given in [8]. These developments provide the basis for further connections, which turn out to be most complete in the setting of triangulated categories. For abelian categories we obtain strong connections in the hereditary case. In fact, we show that when $\mathcal{C}$ is hereditary, then $\mathcal{C}$ has Serre duality if and only if it has almost split sequences and there is a one-one correspondence between indecomposable projective objects $P$ and indecomposable injective objects $I$, such that $P$ modulo its unique maximal subobject is isomorphic to the socle of $I$.

I.1. Preliminaries on Serre duality. Let $\mathcal{A}$ be a $k$-linear Hom-finite additive category. A right Serre functor is an additive functor $F: \mathcal{A} \rightarrow \mathcal{A}$ together with isomorphisms

$$
\eta_{A, B}: \operatorname{Hom}(A, B) \rightarrow \operatorname{Hom}(B, F A)^{*}
$$

for any $A, B \in \mathcal{A}$ which are natural in $A$ and $B$. A left Serre functor is a functor $G: \mathcal{A} \rightarrow \mathcal{A}$ together with isomorphisms

$$
\zeta_{A, B}: \operatorname{Hom}(A, B) \rightarrow \operatorname{Hom}(G B, A)^{*}
$$

for any $A, B \in \mathcal{A}$ which are natural in $A$ and $B$. Below we state and prove a number of properties of right Serre functors. We leave the proofs of the corresponding properties for left Serre functors to the reader. 
Let $\eta_{A}: \operatorname{Hom}(A, F A) \rightarrow k$ be given by $\eta_{A, A}\left(\operatorname{id}_{A}\right)$, and let $f \in \operatorname{Hom}(A, B)$. Looking at the commutative diagram (which follows from the naturality of $\eta_{A, B}$ in B)

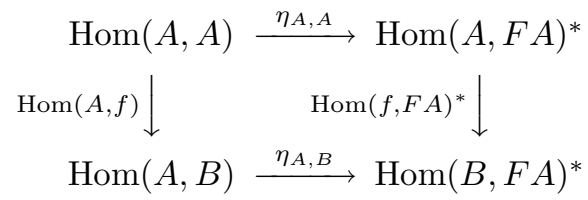

we find for $g \in \operatorname{Hom}(B, F A)$ that

$$
\eta_{A, B}(f)(g)=\eta_{A}(g f)
$$

Similarly by the naturality of $\eta_{A, B}$ in $A$ we obtain the following commutative diagram:

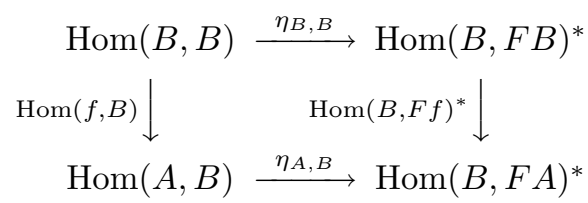

This yields for $g \in \operatorname{Hom}(B, F A)$ the formula

$$
\eta_{A, B}(f)(g)=\eta_{B}(F(f) g)
$$

and we get the following description of the functor $F$.

Lemma I.1.1. The following composition coincides with $F$ :

$$
\operatorname{Hom}(A, B) \stackrel{\eta_{A, B}}{\longrightarrow} \operatorname{Hom}(B, F A)^{*} \stackrel{\left(\eta_{B, F A}^{*}\right)^{-1}}{\longrightarrow} \operatorname{Hom}(F A, F B) .
$$

Proof. To prove this we need to show that for $f \in \operatorname{Hom}(A, B)$ and $g \in \operatorname{Hom}(B, F A)$ one has $\eta_{A, B}(f)(g)=\eta_{B, F A}^{*}(F f)(g)$. Thanks to the formulas (I.1.3), (I.1.4) we obtain $\eta_{A, B}(f)(g)=\eta_{B}(F(f) g)$ and also $\eta_{B, F A}^{*}(F f)(g)=\eta_{B, F A}(g)(F f)=\eta_{B}(F(f) g)$. Thus we obtain indeed the correct result.

We have the following immediate consequence.

Corollary I.1.2. If $F$ is a right Serre functor, then $F$ is fully faithful.

Also note the following basic properties.

Lemma I.1.3. $\quad$ 1. If $F$ and $F^{\prime}$ are right Serre functors, then they are naturally isomorphic.

2. $\mathcal{A}$ has a right Serre functor if and only if $\operatorname{Hom}(A,-)^{*}$ is representable for all $A \in \mathcal{A}$.

From the above discussion it follows that there is a lot of redundancy in the data $\left(F,\left(\eta_{A, B}\right)_{A, B}\right)$. In fact we have the following.

Proposition I.1.4. In order to give $\left(F,\left(\eta_{A, B}\right)_{A, B}\right)$ it is necessary and sufficient to give the action of $F$ on objects, as well as $k$-linear maps $\eta_{A}: \operatorname{Hom}(A, F A) \rightarrow k$ such that the composition

$$
\operatorname{Hom}(A, B) \times \operatorname{Hom}(B, F A) \rightarrow \operatorname{Hom}(A, F A) \stackrel{\eta_{A}}{\longrightarrow} k
$$

yields a non-degenerate pairing for all $A, B \in \mathcal{A}$. If we are given $\eta_{A}$, then $\eta_{A, B}$ is obtained from the pairing (I.1.5). Furthermore the action $F$ on maps

$$
F: \operatorname{Hom}(A, B) \rightarrow \operatorname{Hom}(F A, F B)
$$


is defined by the property that for $f \in \operatorname{Hom}(A, B)$ we have $\eta_{A}(g f)=\eta_{B}(F(f) g)$ for all $g \in \operatorname{Hom}(B, F A)$.

Proof. It is clear from the previous discussion that the data $\left(F,\left(\eta_{A, B}\right)_{A, B}\right)$ gives rise to $\left(\eta_{A}\right)_{A}$ with the required properties. So conversely assume that we are given $\left(\eta_{A}\right)_{A}$ and the action of $F$ on objects. We define $\left(\eta_{A, B}\right)_{A, B}$ and the action of $F$ on maps as in the statement of the proposition.

We first show that $F$ is a functor. Indeed let $A, B, C \in \mathcal{A}$ and assume that there are maps $g: A \rightarrow B$ and $h: B \rightarrow C$. Then for all $f \in \operatorname{Hom}(C, F A)$ we have $\eta_{A}(f h g)=\eta_{C}(F(h g) f)$, but also $\eta_{A}(f h g)=\eta_{B}(F(g) f h)=\eta_{C}(F(h) F(g) f)$. Thus by non-degeneracy we have $F(h g)=F(h) F(g)$.

It is easy to see that the pairing (I.1.5) defines an isomorphism

$$
\eta_{A, B}: \operatorname{Hom}(A, B) \rightarrow \operatorname{Hom}(B, F A)^{*}: f \mapsto \eta_{A}(-f)
$$

which is natural in $A$ and $B$.

The proof is now complete.

A Serre functor is by definition a right Serre functor which is essentially surjective. The following is easy to see.

Lemma 1.1.5. $\mathcal{A}$ has a Serre functor if and only it has both a right and a left Serre functor.

From this we deduce the following [8].

Lemma I.1.6. $\mathcal{A}$ has a Serre functor if and only if the functors $\operatorname{Hom}(A,-)^{*}$ and $\operatorname{Hom}(-, A)^{*}$ are representable for all $A \in \mathcal{A}$.

Remark I.1.7. In the sequel $\mathcal{A}$ will always be a Krull-Schmidt category (in the sense that indecomposable objects have local endomorphism rings). In that case it is clearly sufficient to specify $\eta_{A, B}, \eta_{A}, F$, etc. on the full subcategory of $\mathcal{A}$ consisting of indecomposable objects.

If one is given a right Serre functor, then it is possible to invert it formally in such a way that the resulting additive category has a Serre functor. The next result is stated in somewhat greater generality.

Proposition I.1.8. Let $\mathcal{A}$ be an additive category as above, and let $U: \mathcal{A} \rightarrow \mathcal{A}$ be a fully faithful additive endofunctor. Then there exists an additive category $\mathcal{B}$ with the following properties:

1. There is a fully faithful functor $i: \mathcal{A} \rightarrow \mathcal{B}$.

2. There is an autoequivalence $\bar{U}: \mathcal{B} \rightarrow \mathcal{B}$ together with a natural isomorphism $\nu: \bar{U} i \rightarrow i U$.

3. For every object $B \in \mathcal{B}$ there is some $b \in \mathbb{N}$ such that $\bar{U}^{b} B$ is isomorphic to $i(A)$ with $A \in \mathcal{A}$.

Furthermore a quadruple $(\mathcal{B}, i, \bar{U}, \nu)$ with these properties is unique (in the appropriate sense).

Proof. Let us sketch the construction of $\mathcal{B}$. The uniqueness will be clear.

The objects in $\mathcal{B}$ are formally written as $U^{-a} A$ with $A \in \mathcal{A}$ and $a \in \mathbb{Z}$. A morphism $U^{-a} A \rightarrow U^{-b} B$ is formally written as $U^{-c} f$ with $f \in \operatorname{Hom}_{\mathcal{A}}\left(U^{c-a} A, U^{c-b} B\right)$ where $c \in \mathbb{Z}$ is such that $c \geq a, c \geq b$. We identify $U^{-c} f$ with $U^{-c-1}(U f)$.

The functor $i$ is defined by $i(A)=U^{0} A$ and the functor $\bar{U}$ is defined by $\bar{U}\left(U^{-a}\right)(A)$ $=U^{-a+1}(A)$. It is clear that these have the required properties. 
The following lemma provides a complement to this proposition in the case that $\mathcal{A}$ is triangulated.

Lemma I.1.9. Assume that in addition to the usual hypotheses one has that $\mathcal{A}$ is triangulated. Let $(\mathcal{B}, i, \bar{U}, \nu)$ be as in the previous proposition. Then there is a unique way to make $\mathcal{B}$ into a triangulated category such that $i$ and $\bar{U}$ are exact.

Proof. If we require exactness of $i$ and $\bar{U}$, then there is only one way to make $\mathcal{B}$ into a triangulated category. First we must define the shift functor by $\left(U^{-a} A\right)[1]=$ $U^{-a}(A[1])$ and then the triangles in $\mathcal{B}$ must be those diagrams that are isomorphic to

$$
U^{-c} A \stackrel{U^{-c} f}{\longrightarrow} U^{-c} B \stackrel{U^{-c} g}{\longrightarrow} U^{-c} C \stackrel{U^{-c} h}{\longrightarrow} U^{-c} A[1]
$$

where

$$
A \stackrel{f}{\rightarrow} B \stackrel{g}{\rightarrow} C \stackrel{h}{\rightarrow} A[1]
$$

is a triangle in $\mathcal{A}$ (note that the exactness of $\bar{U}$ is equivalent to that of $\bar{U}^{-1}$ ).

To show that this yields indeed a triangulated category one must check the axioms in [34]. These all involve the existence of certain objects/maps/triangles. By applying a sufficiently high power of $\bar{U}$ we can translate such problems into ones involving only objects in $\mathcal{A}$. Then we use the triangulated structure of $\mathcal{A}$ and afterwards we go back to the original problem by applying a negative power of $\bar{U}$.

In the sequel we will denote by $U^{-\infty} \mathcal{A}$ the category $\mathcal{B}$ which was constructed in Proposition [1.1.8. Furthermore we will consider $\mathcal{A}$ as a subcategory of $U^{-\infty} \mathcal{A}$ through the functor $i$. Finally we will usually write $U$ for the extended functor $\bar{U}$.

Below we will only be interested in the special case where $U=F$ is a right Serre functor on $\mathcal{A}$. In that case we have the following.

Proposition I.1.10. The canonical extension of $F$ to $F^{-\infty} \mathcal{A}$ is a Serre functor.

Proof. By construction $F$ is an automorphism on $F^{-\infty} \mathcal{A}$. To prove that $F$ is a Serre functor we have to construct suitable maps $\eta_{F^{-a} A, F^{-b} B}$. Pick $c \geq a, c \geq b$. Then we have

$$
\begin{aligned}
& \operatorname{Hom}_{F^{-\infty}} \mathcal{A}\left(F^{-a} A, F^{-b} B\right)=\operatorname{Hom}_{\mathcal{A}}\left(F^{c-a} A, F^{c-b} B\right) \\
& \eta_{F^{c-a}} \stackrel{A, F^{c-b} B}{\cong} \operatorname{Hom}_{\mathcal{A}}\left(F^{c-b} B, F^{c-a+1} A\right)^{*} \\
& =\operatorname{Hom}_{F^{-\infty} \mathcal{A}}\left(F^{-b} B, F^{-a+1} A\right)^{*} \text {. }
\end{aligned}
$$

We define $\eta_{F^{-a} A, F^{-b} B}$ as the composition of these maps. It follows easily from Lemma I.1.1 that the constructed map is independent of $c$, and it is clear that $\eta_{F^{-a} A, F^{-b} B}$ has the required properties.

We shall also need the following easily verified fact.

Lemma I.1.11. If $\mathcal{A}=\mathcal{A}_{1} \oplus \mathcal{A}_{2}$ is a direct sum of additive categories, then a (right) Serre functor on $\mathcal{A}$ restricts to (right) Serre functors on $\mathcal{A}_{1}$ and $\mathcal{A}_{2}$. 
I.2. Connection between Serre duality and Auslander-Reiten triangles. In this section we prove that existence of a right Serre functor is equivalent to the existence of right Auslander-Reiten triangles, in triangulated Hom-finite KrullSchmidt $k$-categories. Hence the existence of a Serre functor is equivalent to the existence of Auslander-Reiten triangles.

In the sequel $\mathcal{A}$ is a Hom-finite $k$-linear Krull-Schmidt triangulated category. Following [14] a triangle $A \stackrel{f}{\rightarrow} B \stackrel{g}{\rightarrow} C \stackrel{h}{\rightarrow} A[1]$ in $\mathcal{A}$ is called an Auslander-Reiten triangle if the following conditions are satisfied:

(AR1) $A$ and $C$ are indecomposable.

(AR2) $h \neq 0$.

(AR3) If $D$ is indecomposable, then for every non-isomorphism $t: D \rightarrow C$ we have $h t=0$.

It is shown in 14 that, assuming (AR1) and (AR2), then (AR3) is equivalent to

$(\mathrm{AR} 3)^{\prime}$ If $D^{\prime}$ is indecomposable, then for every non-isomorphism $s: A \rightarrow D^{\prime}$ we have $s h[-1]=0$.

We say that right Auslander-Reiten triangles exist in $\mathcal{A}$ if for all indecomposables $C \in \mathcal{A}$ there is a triangle satisfying the conditions above. Existence of left Auslander-Reiten triangles is defined in a similar way, and we say that $\mathcal{A}$ has Auslander-Reiten triangles if it has both right and left Auslander-Reiten triangles. (Note that in 14 one says that $\mathcal{A}$ has Auslander-Reiten triangles if it has right Auslander-Reiten triangles in our terminology.)

It is shown in [14, §4.3] that given $C$ the corresponding Auslander-Reiten triangle $A \rightarrow B \rightarrow C \rightarrow A[1]$ is unique up to isomorphism of triangles. (By duality a similar result holds if $A$ is given.) For a given indecomposable $C$ we let $\tilde{\tau} C$ be an arbitrary object in $\mathcal{A}$, isomorphic to $A$ in the Auslander-Reiten triangle corresponding to $C$.

The following characterization of Auslander-Reiten triangles is analogous to the corresponding result on almost split sequences (see [5]).

Proposition I.2.1. Assume that $\mathcal{A}$ has right Auslander-Reiten triangles, and assume that we have a triangle in $\mathcal{A}$

$$
A \stackrel{f}{\rightarrow} B \stackrel{g}{\rightarrow} C \stackrel{h}{\rightarrow} A[1]
$$

with $A$ and $C$ indecomposable and $h \neq 0$. Then the following are equivalent:

1. The triangle (1.2.1) is an Auslander-Reiten triangle.

2. The map $h$ is in the socle of $\operatorname{Hom}(C, A[1])$ as a right $\operatorname{End}(C)$-module and $A \cong \tilde{\tau} C$.

3. The map $h$ is in the socle of $\operatorname{Hom}(C, A[1])$ as a left $\operatorname{End}(A)$-module and $A \cong$ $\tilde{\tau} C$.

Proof. We will show that 1 . and 2. are equivalent. The equivalence of 1 . and 3 . is similar.

1. $\Rightarrow 2$. By definition we have $A \cong \tilde{\tau} C$. Assume that $t \in \operatorname{End}(C)$ is a nonautomorphism. Then by (AR3) we have $h t=0$.

$2 . \Rightarrow 1$. Let

$$
A \stackrel{f^{\prime}}{\longrightarrow} B^{\prime} \stackrel{g^{\prime}}{\longrightarrow} C \stackrel{h^{\prime}}{\longrightarrow} A[1]
$$


be the Auslander-Reiten triangle associated to $C$. From the properties of Auslander-Reiten triangles it follows that there is a morphism of triangles

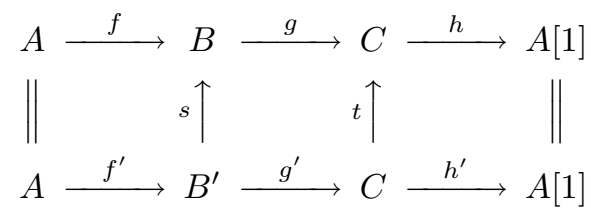

The fact that $h^{\prime} \neq 0$ together with the fact that $h$ is in the socle of $\operatorname{Hom}(C, A[1])$ implies that $t$ must be an isomorphism. But then by the properties of triangles $s$ is also an isomorphism. So in fact the triangles (I.2.2) and (I.2.1) are isomorphic, and hence in particular (I.2.1) is an Auslander-Reiten triangle.

Corollary I.2.2. Assume that $\mathcal{A}$ has right Auslander-Reiten triangles. Then the socle of $\operatorname{Hom}(C, \tilde{\tau} C[1])$ is one-dimensional both as a right $\operatorname{End}(C)$-module and as a left $\operatorname{End}(\tilde{\tau} C)$-module.

Proof. It is easy to see that $u$ linearly independent elements of the (left or right) socle define different triangles. However Auslander-Reiten triangles are unique. This is a contradiction.

The following is the basis for the main result of this section.

Proposition I.2.3. The following are equivalent:

1. $\mathcal{A}$ has a right Serre functor.

2. $\mathcal{A}$ has right Auslander-Reiten triangles.

If either of these properties holds, then the action of the Serre functor on objects coincides with $\tilde{\tau}[1]$.

Proof.

1. $\Rightarrow 2$. Let $C \in \mathcal{A}$ be an indecomposable object. By Serre duality there is a natural isomorphism

$$
\operatorname{Hom}(C, F C) \cong \operatorname{Hom}(C, C)^{*}
$$

as $\operatorname{End}(C)$-bimodules. In particular $\operatorname{Hom}(C, F C)$ has a one dimensional socle which corresponds to the map $\theta_{C}: \operatorname{Hom}(C, C) \rightarrow \operatorname{Hom}(C, C) / \operatorname{rad}(C, C)$ $=k$. Define $\tilde{\tau} C=F C[-1]$, and let $h$ be a non-zero element of the socle of $\operatorname{Hom}(C, \tilde{\tau} C[1])$. We claim that the associated triangle

$$
\tilde{\tau} C \rightarrow X \rightarrow C \stackrel{h}{\rightarrow} \tilde{\tau} C[1]
$$

is an Auslander-Reiten triangle. Let $D$ be indecomposable, and let $t$ : $D \rightarrow C$ be a non-isomorphism. We have to show that the composition

$$
D \stackrel{t}{\rightarrow} C \stackrel{h}{\longrightarrow} F C
$$

is zero. Using Serre duality this amounts to showing that the composition

$$
\operatorname{Hom}(C, D) \stackrel{\operatorname{Hom}(C, t)}{\longrightarrow} \operatorname{Hom}(C, C) \stackrel{\theta_{C}}{\longrightarrow} k
$$

is zero. Since $t$ is a non-isomorphism, this is clear. 
2. $\Rightarrow 1$. This is the interesting direction. As pointed out in Remark I.1.7 it is sufficient to construct the Serre functor on the full subcategory of $\mathcal{A}$ consisting of the indecomposable objects. For $A$ an indecomposable object in $\mathcal{A}$ we let $F A$ be the object $\tilde{\tau} A[1]$. Let $h_{A}$ be a non-zero element of $\operatorname{Hom}(A, \tilde{\tau} A[1])$ representing the Auslander-Reiten triangle

$$
\tilde{\tau} A \rightarrow X \rightarrow A \stackrel{h_{A}}{\longrightarrow} \tilde{\tau} A[1] .
$$

Sublemma. Let $A$ and $B$ be indecomposable objects in $\mathcal{C}$. Then the following hold:

1. For any non-zero $f \in \operatorname{Hom}(B, \tilde{\tau} A[1])$ there exists $g \in \operatorname{Hom}(A, B)$ such that $f g=h_{A}$.

2. For any non-zero $g \in \operatorname{Hom}(A, B)$ there exists $f \in \operatorname{Hom}(B, \tilde{\tau} A[1])$ such that $f g=h_{A}$.

Proof. 1. Using the properties of Auslander-Reiten triangles there is a morphism between the triangle determined by $h_{A}$ (the AR-triangle) and the triangle determined by $f$.

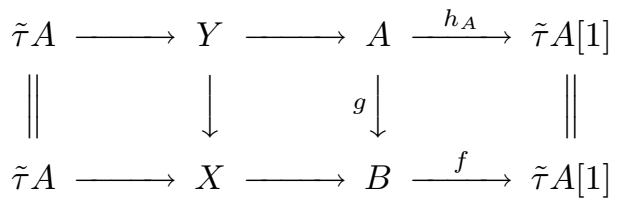

The morphism labeled $g$ in the above diagram has the required properties.

2. Without loss of generality we may assume that $g$ is not an isomorphism. We complete $g$ to a triangle

$$
Z \stackrel{s}{\rightarrow} A \stackrel{g}{\rightarrow} B \stackrel{t}{\rightarrow} Z[1]
$$

Then $Z \neq 0$ and since $g$ is non-zero, $s$ will not be split. Now we look at the following diagram:

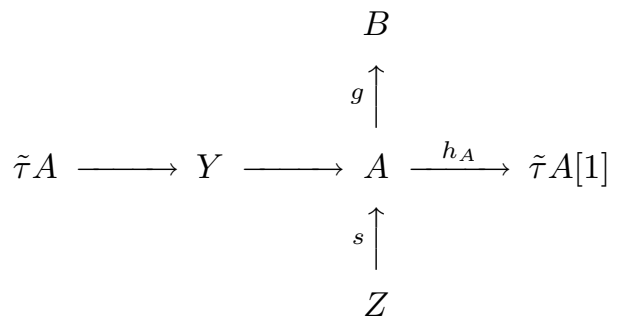

Since $s$ is not split we have by (AR3) that $h_{A} s=0$. Hence by the properties of triangles we have $h_{A}=f g$ for a map $f: B \rightarrow \tau A[1]$. This proves what we want.

Having proved the sublemma we return to the main proof. For any indecomposable object $A \in \mathcal{C}$ choose a linear map

$$
\eta_{A}: \operatorname{Hom}(A, \tilde{\tau} A[1]) \rightarrow k
$$

such that $\eta_{A}\left(h_{A}\right) \neq 0$. It follows from the sublemma that the pairing

$$
\operatorname{Hom}(A, B) \times \operatorname{Hom}(B, \tilde{\tau} A[1]) \rightarrow k \text { given by }(g, f) \rightarrow \eta_{A}(f g)
$$

is non-degenerate. We can now finish our proof by invoking Proposition I.1.4

The following is now a direct consequence. 
Theorem I.2.4. The following are equivalent:

1. $\mathcal{A}$ has a Serre functor.

2. $\mathcal{A}$ has Auslander-Reiten triangles.

Proof. This follows from applying Proposition $\amalg .2 .3$ together with its dual version for left Serre functors.

From now on we shall denote by $\tilde{\tau}$ also the equivalence $F[-1]$, where $F$ is the Serre functor.

I.3. Serre functors on hereditary abelian categories. In this section we investigate the relationship between existence of Serre functors and of almost split sequences for hereditary abelian categories.

If $\mathcal{C}$ is an Ext-finite $k$-linear abelian category, we say that it has a right Serre functor if this is the case for $D^{b}(\mathcal{C})$.

If $\mathcal{C}$ has a right Serre functor $F$ and $A$ and $B$ are in $\mathcal{C}$, then from the fact that $\operatorname{Ext}^{i}(A, B)=\operatorname{Hom}(A, B[i])=\operatorname{Hom}(B[i], F A)^{*}$ we deduce that only a finite number of $\operatorname{Ext}^{i}(A, B)$ can be non-zero.

Before we go on we recall some basic definitions (see [4]). For an indecomposable object $C$ in $\mathcal{C}$ a map $g: B \rightarrow C$ is right almost split if for any non-isomorphism $h: X \rightarrow C$ with $X$ indecomposable in $\mathcal{C}$ there is some map $t: B \rightarrow C$ with $g t=h$. The map $g: B \rightarrow C$ is minimal right almost split if in addition $g: B \rightarrow C$ is right minimal, that is, any map $s: B \rightarrow B$ with $g s=g$ is an isomorphism. The concepts of left almost split and minimal left almost split are defined similarly. A non-split exact sequence $0 \rightarrow A \rightarrow B \rightarrow C \rightarrow 0$ is almost split if $A$ and $C$ are indecomposable and $g: B \rightarrow C$ is (minimal) right almost split (or equivalently, $f: A \rightarrow B$ is (minimal) left almost split). We say that $\mathcal{C}$ has right almost split sequences if for every non-projective indecomposable object $C \in \mathcal{C}$ there exists an almost split sequence ending in $C$, and for each indecomposable projective object $P$ there is a minimal right almost split map $E \rightarrow P$. Possession of left almost split sequences is defined similarly. We say that $\mathcal{C}$ has almost split sequences if it has both left and right almost split sequences.

Now let $\mathcal{C}$ be an Ext-finite $k$-linear hereditary abelian category.

The following characterization of when we have a minimal right almost split map to a projective object or a minimal left almost split map from an injective object is easy to see.

Lemma I.3.1. $\quad$ 1. There is some minimal right almost split map to an indecomposable projective object $P$ if and only if $P$ has a unique maximal subobject $\operatorname{rad} P$. (If the conditions are satisfied, then the inclusion map $i: \operatorname{rad} P \rightarrow P$ is minimal right almost split.)

2. There is a minimal left almost split map from an indecomposable injective object I if and only if I has a unique simple subobject $S$. (If these conditions are satisfied, then the epimorphism $j: I \rightarrow I / S$ is minimal left almost split.)

Note that if an indecomposable projective object has a maximal subobject, then it must be unique, and if $P$ is noetherian, then $P$ has a maximal subobject. Similarly if an indecomposable injective object $I$ has a simple subobject, then it must be unique.

Lemma I.3.2. Let $\mathcal{A}=D^{b}(\mathcal{C})$. The following are equivalent:

1. $\mathcal{A}$ has right Auslander-Reiten triangles. 
2. $\mathcal{C}$ has right almost split sequences and for every indecomposable projective $P \in \mathcal{C}$ the simple object $S=P / \operatorname{rad}(P)$ possesses an injective hull I in $\mathcal{C}$.

3. $\mathcal{C}$ has a right Serre functor.

If any of these conditions holds, then the right Auslander-Reiten triangles in $\mathcal{A}$ are given by the shifts of the right almost split sequences in $\mathcal{C}$ together with the shifts of the triangles of the form

$$
I[-1] \rightarrow X \rightarrow P \stackrel{h_{P}}{\longrightarrow} I
$$

where $I$ and $P$ are as in 2 . and $h_{P}$ is the composition $P \rightarrow S \rightarrow I$. The middle term $X$ of this triangle is isomorphic to $I / S[-1] \oplus \operatorname{rad} P$.

Proof. This follows using Proposition I.2.3 and standard properties of almost split sequences and Auslander-Reiten triangles. We leave the proof to the reader.

We now get the following main result on the connection between Serre duality and almost split sequences.

Theorem I.3.3. Let $\mathcal{C}$ be an Ext-finite hereditary abelian category.

1. $\mathcal{C}$ has Serre duality if and only if $\mathcal{C}$ has almost split sequences, and there is a one-one correspondence between indecomposable projective objects $P$ and indecomposable injective objects $I$, via $P / \operatorname{rad} P \cong \operatorname{soc} I$.

2. If $\mathcal{C}$ has no non-zero projective or injective objects, then $\mathcal{C}$ has Serre duality if and only if it has almost split sequences.

Note that if $\mathcal{C}$ is the category of finite dimensional representations over $k$ of the quiver $\rightarrow \cdot \rightarrow \cdot \rightarrow \cdots$, then $\mathcal{C}$ is an Ext-finite hereditary abelian $k$-category with almost split sequences. Since $\mathcal{C}$ has non-zero injective objects, but no non-zero projective objects, it does not have Serre duality.

Let $\mathcal{P}$ and $\mathcal{I}$ be the full subcategories of $\mathcal{C}$ whose objects are respectively the projectives and the injectives in $\mathcal{C}$. If $H$ is a set of objects in $\mathcal{C}$, then we denote by $\mathcal{C}_{H}$ the full subcategory of $\mathcal{C}$ whose objects have no summands in $H$.

When $\mathcal{C}=\bmod A$ for a finite dimensional hereditary $k$-algebra $A$, we have an equivalence $\tau: \mathcal{C}_{\mathcal{P}} \rightarrow \mathcal{C}_{\mathcal{I}}$, where for an indecomposable object $C$ in $\mathcal{C}$ the object $\tau C$ is the left hand term of the almost split sequence with right hand term $C$. Also we have the Nakayama functor $N: \mathcal{P} \rightarrow \mathcal{I}$, which is an equivalence of categories, where $N(P)=\operatorname{Hom}_{k}\left(\operatorname{Hom}_{A}(P, A), k\right)$ for $P \in \mathcal{P}$. For the equivalence $F: D^{b}(\mathcal{C}) \rightarrow D^{b}(\mathcal{C})$, where $F$ is the Serre functor, we have $F \mid \mathcal{C}_{\mathcal{P}}=\tau[1]$ and $F \mid \mathcal{P}=N$. Hence $F$ is in some sense put together from the two equivalences $\tau$ and $N$ (see [14]). Using Lemma I.3.2 we see that the situation is similar in the general case.

Corollary I.3.4. Assume that $\mathcal{C}$ has a right Serre functor $F$. Then the following hold:

1. F defines a fully faithful functor $\mathcal{P} \rightarrow \mathcal{I}$. We denote this functor by $N$ (the "Nakayama functor").

2. $F[-1]=\tilde{\tau}$ induces a fully faithful functor $\mathcal{C}_{\mathcal{P}} \rightarrow \mathcal{C}_{\mathcal{I}}$, which we denote by $\tau$.

3. If $P \in \mathcal{P}$ is indecomposable, then $\operatorname{Hom}(P, N P)$ has one dimensional socle, both as left $\operatorname{Hom}(N P, N P)$-module and as right $\operatorname{Hom}(P, P)$-module. Let $h_{P}$ be a non-zero element in this socle. Then $S=\operatorname{im} h_{P}$ is simple, and furthermore $P$ is a projective cover of $S$ and $N P$ is an injective hull of $S$.

If $F$ is a Serre functor, then the functors $N$ and $\tau$ defined above are equivalences. 
Proof. That $F$ takes the indicated values on objects follows from the nature of the Auslander-Reiten triangles in $D^{b}(\mathcal{C})$ (given by Lemma I.3.2). The assertion about fully faithfulness of $\tau$ and $N$ follows from the corresponding property of $F$. Part 3 . follows by inspecting the triangle (I.3.1). Finally that $N$ and $\tau$ are equivalences in the case that $F$ is a Serre functor follows by considering $F^{-1}$, which is a left Serre functor.

In the next section we will use the following result.

Lemma I.3.5. Assume that $\mathcal{A}$ is a triangulated category with a t-structure $\left(\mathcal{A}_{\leq 0}\right.$, $\left.\mathcal{A}_{\geq 0}\right)$ in such a way that every object in $\mathcal{A}$ lies in some $\mathcal{A}^{[a, b]}$. Let $\mathcal{C}$ be the heart of the t-structure, and assume that $\operatorname{Hom}(A, B[n])=0$ for $A, B \in \mathcal{C}$ and $n \neq 0,1$. Then $\mathcal{C}$ is a hereditary abelian category, and furthermore $\mathcal{A}$ has a (right) Serre functor if and only if $\mathcal{C}$ has a (right) Serre functor.

Proof. To show that $\mathcal{C}$ is hereditary we have to show that

$$
\operatorname{Ext}_{\mathcal{C}}^{n}(A, B)=\operatorname{Hom}_{\mathcal{A}}(A, B[n]) .
$$

Now $\operatorname{Ext}_{\mathcal{C}}^{m}(A,-)$ is characterized by the property that it is an effaceable $\delta$-functor which coincides in degree zero with $\operatorname{Hom}_{\mathcal{C}}(A,-)$. Hence we have to show that $\operatorname{Hom}_{\mathcal{A}}(A,-[n])$ is effaceable. This is clear in degree $\geq 2$ since there the functor is zero, and for $n \leq 1$ it is also clear since then $\operatorname{Hom}_{\mathcal{A}}(A,-[n])=\operatorname{Ext}_{\mathcal{C}}^{n}(A,-)$ [7] p. 75].

Now standard arguments show that as additive categories $\mathcal{A}$ and $D^{b}(\mathcal{C})$ are equivalent to $\bigoplus_{n} \mathcal{C}[n]$. We don't know if this equivalence yields an exact equivalence between $\mathcal{A}$ and $D^{b}(\mathcal{C})$, but recall that the definition of a (right) Serre functor does not involve the triangulated structure. Hence if $\mathcal{A}$ has a (right) Serre functor, then so does $D^{b}(\mathcal{C})$ and vice versa.

\section{HeREditary NOETHERIAN ABELIAN CATEGORIES WITH NON-ZERO PROJECTIVE OBJECTS}

In this section we classify the connected noetherian hereditary Ext-finite abelian categories with Serre functor, in the case where there are non-zero projective objects.

Let $\mathcal{C}$ be a connected hereditary abelian noetherian Ext-finite category with Serre functor and non-zero projective objects. The structure of the projective objects gives rise to an associated quiver $Q$, which satisfies special assumptions, including being locally finite and having no infinite path ending at any vertex. The category $\mathcal{C}$ contains the category $\operatorname{rep} Q$ of finitely presented representations of $Q$ as a full subcategory, which may be different from $\mathcal{C}$. Actually $\operatorname{rep} Q$ is the full subcategory generated by projective objects. Hence $\mathcal{C}$ is not in general generated by the projective objects, but it is generated by the preprojective objects. This provides a new interesting phenomenon.

Conversely, starting with a locally finite quiver $Q$ having no infinite path ending at any vertex, the category $\operatorname{rep} Q$ is a hereditary abelian Ext-finite category with a right Serre functor (and right almost split sequences), but not necessarily having a Serre functor. We construct a hereditary abelian Ext-finite category with Serre functor $\widetilde{\operatorname{rep}}(Q)$ containing rep $Q$ as a full subcategory. We describe for which quivers $Q$ the category $\widetilde{\operatorname{rep}}(Q)$ is noetherian, and show that in the noetherian case all 
connected noetherian hereditary abelian Ext-finite categories with Serre duality are of this form.

In $₫$ II.1 we explain the construction of $\widetilde{\mathrm{eep}}(Q)$ via inverting a right Serre functor. An alternative approach using derived categories is discussed in $\$ 1.3$ After learning about our results Claus Ringel found yet another approach towards the construction of $\widetilde{\mathrm{rep}}(Q)[24$.

In $\amalg$ II. we show that if $\mathcal{C}$ is generated by the preprojective objects, then $\mathcal{C}$ is equivalent to some $\widetilde{\text { rep }}(Q)$. That $\mathcal{C}$ is generated by preprojective objects when $\mathcal{C}$ is connected and noetherian is proved in Section \I.4, along with showing for which quivers $Q$ the category $\widetilde{\text { rep }}(Q)$ is noetherian.

II.1. Hereditary abelian categories constructed from quivers. For a quiver $Q$ denote by $\operatorname{rep}(Q)$ the category of finitely presented representations of $Q$. Under some additional assumptions on $Q$ we construct a hereditary abelian Ext-finite category $\widetilde{\operatorname{rep}}(Q)$ with Serre functor. The category $\widetilde{\operatorname{rep}}(Q)$ contains $\operatorname{rep}(Q)$ as a full subcategory, and is obtained from $\operatorname{rep}(Q)$ by formally inverting a right Serre functor.

Let $Q$ be a quiver with the following properties:

(P1) $Q$ is locally finite, that is, every vertex in $Q$ is adjacent to only a finite number of other vertices.

(P2) There is no infinite path in $Q$ of the form $x_{0} \leftarrow x_{1} \leftarrow x_{2} \leftarrow \cdots$ (in particular there are no oriented cycles).

Note that (P1), (P2) imply the following.

Lemma II.1.1. $\quad$ 1. If $x$ is a vertex in $Q$, then there are only a finite number of paths ending in $x$.

2. If $x$ and $y$ are vertices in $Q$, then there are only a finite number of paths from $x$ to $y$.

Proof. Part 2. is an obvious consequence of 1., so we prove 1. Assume there is an infinite number of paths ending in $x$. By (P1) there must be an arrow $x_{1} \rightarrow x$ such that there is an infinite number of paths ending in $x_{1}$. Repeating this we obtain an infinite path $\cdots \rightarrow x_{2} \rightarrow x_{1} \rightarrow x$ such that there is an infinite number of paths ending in every $x_{n}$. The existence of such an infinite path contradicts (P2).

For a vertex $x$ in $Q$ we denote by $P_{x}, I_{x}$ and $S_{x}$ respectively the corresponding projective, injective and simple object in $\operatorname{Rep}(Q)$, the category of all $Q$-representations. By Lemma II.1.1 the objects $I_{x}$ have finite length. We also have a canonical isomorphism

$$
\operatorname{Hom}\left(P_{x}, P_{y}\right) \cong \operatorname{Hom}\left(I_{x}, I_{y}\right)
$$

since both of these vector spaces have as basis the paths from $y$ to $x$.

The category $\operatorname{Rep}(Q)$ is too big for what we want. For example it is not Extfinite. As will be clear from the considerations in the next section the natural subcategory to consider is $\operatorname{rep}(Q)$. It is easy to see that this is a hereditary abelian subcategory of $\operatorname{Rep}(Q)$, and furthermore by Lemma I.1.1 it has finite dimensional Ext's. Thanks to (P1) the simple and hence the finite dimensional representations are contained in $\operatorname{rep}(Q)$. Hence in particular $I_{x} \in \operatorname{rep}(Q)$ for every vertex $x$.

Let $\mathcal{P}$ and $\mathcal{I}$ be the full subcategories of $\operatorname{rep}(Q)$ consisting respectively of the finite direct sums of the $P_{x}$ and the finite direct sums of the $I_{x}$. Let $N: \mathcal{P} \rightarrow \mathcal{I}$ be the equivalence obtained from additively extending (II.1.1). We denote also by 
$N$ the corresponding equivalence $N: K^{b}(\mathcal{P}) \rightarrow K^{b}(\mathcal{I})$. Finally denoting by $F$ the composition

$$
D^{b}(\operatorname{rep}(Q)) \cong K^{b}(\mathcal{P}) \stackrel{N}{\longrightarrow} K^{b}(\mathcal{I}) \rightarrow D^{b}(\operatorname{rep}(Q))
$$

we have the following.

Lemma II.1.2. $F$ is a right Serre functor.

Proof. Let $A, B \in K^{b}(\mathcal{P})$. Then we need to construct natural isomorphisms

$$
\operatorname{Hom}_{K^{b}(\mathcal{P})}(A, B) \rightarrow \operatorname{Hom}_{D^{b}(\operatorname{rep}(Q))}(B, F A)^{*} .
$$

Since $A$ and $B$ are finite complexes of projectives we can reduce to the case $A=P_{x}$ and $B=P_{y}$. So we need natural isomorphisms

$$
\operatorname{Hom}\left(P_{x}, P_{y}\right) \rightarrow \operatorname{Hom}\left(P_{y}, I_{x}\right)^{*}
$$

Again both of these vector spaces have a natural basis given by the paths from $y$ to $x$. This leads to the required isomorphisms.

Under the assumptions (P1), (P2) on the quiver $Q$ we now know that $\operatorname{rep} Q$ is a hereditary abelian Ext-finite category with a right Serre functor $F$ such that the image of the projective objects in $\operatorname{rep} Q$ are injective objects in $\operatorname{rep} Q$ of finite length. Hence the following result applies to this setting.

Theorem II.1.3. Let $\mathcal{B}$ be an abelian Ext-finite hereditary category with a right Serre functor $F$ and enough projectives. Denote the full subcategory of projective objects in $\mathcal{B}$ by $\mathcal{P}$ and assume that $F(\mathcal{P})=\mathcal{I}$ consists of (injective) objects of finite length. Then there exists an Ext-finite abelian hereditary category $\mathcal{C}$ with the following properties:

1. There exists a full faithful exact embedding $i: \mathcal{B} \rightarrow \mathcal{C}$.

2. The injectives and projectives in $\mathcal{C}$ are given by $i(\mathcal{I})$ and $i(\mathcal{P})$.

3. $\mathcal{C}$ possesses a Serre functor which extends $F: D^{b}(\mathcal{B}) \rightarrow D^{b}(\mathcal{B})$ in such a way that there is a natural equivalence $\nu: F i \rightarrow i F$ where we have denoted the derived functor of $i$ also by $i$ and the extended Serre functor also by $F$.

4. For every indecomposable object $X \in \mathcal{C}$ there exists $t \geq 0$ such that $\tau^{t} X$ is defined and lies in $\mathcal{B}$, where $\tau$ denotes the functor $\mathcal{C}_{\mathcal{P}} \rightarrow \mathcal{C}_{\mathcal{I}}$ (see Corollary I.3.4.2) induced by $F[-1]=\tilde{\tau}$.

Furthermore a quadruple $(\mathcal{C}, i, F, \nu)$ with these properties is unique in the appropriate sense.

Proof. First we show that if $\mathcal{C}$ exists satisfying properties 1.,2.,3., 4., then it is unique. This proof will in particular tell us how to construct $\mathcal{C}$. We then show that this construction always yields a category $\mathcal{C}$ with the required properties.

Since $D^{b}(\mathcal{B})=K^{b}(\mathcal{P})$ and since the objects in $\mathcal{P}$ remain projective in $\mathcal{C}$ by property 2., it follows that the derived functor of $i$ is fully faithful. It is also clear that $D^{b}(\mathcal{B})$ is closed inside $D^{b}(\mathcal{C})$ under the formation of cones.

Let $F: D^{b}(\mathcal{C}) \rightarrow D^{b}(\mathcal{C})$ be the extended Serre functor. If $K \in D^{b}(\mathcal{C})$, then $K$ can be obtained by starting with objects in $\mathcal{C}$ and repeatedly taking cones. It then follows from 4 . that for $t \gg 0$ we have $F^{t} K \in D^{b}(\mathcal{B})$. Hence the triple $\left(D^{b}(\mathcal{C}), i, F\right)$ satisfies the hypotheses of Proposition I.1.8. Thus we obtain $D^{b}(\mathcal{C})=F^{-\infty} D^{b}(\mathcal{B})$.

According to 4. every indecomposable object in $\mathcal{C}$ will be of the form $\tau^{-t} Y$ with $Y \in \mathcal{B}$. Implicit in the notation $\tau^{-t} Y$ is the assumption that $\tau^{-l} Y$ is defined for $l=1, \ldots, t$, that is, $\tau^{-i} Y \notin \mathcal{I}$ for $i=0, \ldots, t-1$. In addition we may assume that 
$t$ is minimal. Thus either $t=0$ or else $\tau^{-l} Y \notin \mathcal{B}$ for $l=1, \ldots, t$. The last case is equivalent to

$$
\tau^{-i} Y \notin \tau \mathcal{B}_{\mathcal{P}} \cup \mathcal{I}
$$

for $i=0, \ldots, t-1$.

If $Y \notin \tau \mathcal{B}_{\mathcal{P}} \cup \mathcal{I}$, then the same is true for $\tau^{-1} Y$. Hence we have to impose (11.1.4) only for $i=0$.

We conclude that the indecomposable objects in $\mathcal{C}$ are of the following form:

(C1) The indecomposable objects in $\mathcal{B}$.

(C2) Objects of the form $\tilde{\tau}^{-t} Y$ where $t>0$ and $Y$ is an indecomposable object in $\mathcal{B} \backslash\left(\tau \mathcal{B}_{\mathcal{P}} \cup \mathcal{I}\right)$, where $\tilde{\tau}=F[-1]$.

This completes the determination of $\mathcal{C}$ as an additive subcategory of $F^{-\infty} D^{b}(B)$, and finishes the proof of the uniqueness.

Let us now assume that $\mathcal{C}$ is the additive subcategory of $F^{-\infty} D^{b}(\mathcal{B})$ whose indecomposable objects are given by $(\mathrm{C} 1),(\mathrm{C} 2)$. We have to show that $\mathcal{C}$ is a hereditary abelian category satisfying 1.-4.

Since $F^{-\infty} D^{b}(\mathcal{B})$ has a Serre functor, it has Auslander-Reiten triangles. Below we will need the triangle associated to $P_{x}[1]$. Using the criterion given in Proposition I.2.1 we can compute this triangle in $D^{b}(\mathcal{B})$. So according to Lemma I.3.2 the requested triangle is of the form

$$
I_{x} \rightarrow I_{x} / \operatorname{soc}\left(I_{x}\right) \oplus \operatorname{rad}\left(P_{x}\right)[1] \rightarrow P_{x}[1] \rightarrow .
$$

We now define a $t$-structure on $F^{-\infty} D^{b}(\mathcal{B})$. Using the fact that $\mathcal{B}$ is hereditary we easily obtain that as additive categories $F^{-\infty} D^{b}(\mathcal{B})=\bigoplus_{n} \mathcal{C}[n]$. We now define

$$
\begin{aligned}
F^{-\infty} D^{b}(\mathcal{B})_{\leq 0} & =\bigoplus_{n \geq 0} \mathcal{C}[n], \\
F^{-\infty} D^{b}(\mathcal{B})_{\geq 0} & =\bigoplus_{n \leq 0} \mathcal{C}[n] .
\end{aligned}
$$

We claim that this is a $t$-structure. The only non-trivial axiom we have to verify is that

$$
\operatorname{Hom}\left(F^{-\infty} D^{b}(\mathcal{B})_{\leq 0}, F^{-\infty} D^{b}(\mathcal{B})_{\geq 1}\right)=0 .
$$

So this amounts to showing that $\operatorname{Ext}^{-i}(A, B)=0$ for $A, B \in \mathcal{C}$ and for $i>0$. We separate this into four cases.

Case 1. $A$ and $B$ fall under (C1). This case is trivial.

Case 2. $A$ falls under (C1) and $B$ falls under (C2). Thus we have $B=\tilde{\tau}^{-t} X$ for some $X$ in $\mathcal{B}$ and $t>0$. We want to show that $\operatorname{Ext}^{-i}(A, B)=0$ for $i>0$ for any $A \in \mathcal{B}$, by induction on $t$.

First let $t=1$. We can assume that $A$ is projective, since otherwise we could reduce to Case 1 by applying $\tilde{\tau}$. Then $\tilde{\tau}(A)=I[-1]$ for some object $I$ in $\mathcal{I}$, so it is sufficient to show $\operatorname{Ext}^{-i}(I[-1], X) \cong \operatorname{Ext}^{-(i-1)}(I, X)=0$ for $i>0$. For $i>1$ this follows from Case 1. For $i=1$ we clearly have $\operatorname{Hom}(I, X)=0$ since $I$ is injective in $\mathcal{B}$ and $X$ is not injective.

Assume now that $t>1$ and that the claim has been proved for $t-1$ for all objects $A$ in $\mathcal{B}$. Then we can again assume that $A$ is projective, and consider $\operatorname{Ext}^{-(i-1)}\left(I, \tilde{\tau}^{-t+1} X\right)$, where $\tilde{\tau}(A)=I[-1]$. We want to prove that

$$
\operatorname{Ext}^{-(i-1)}\left(I, \tilde{\tau}^{-t+1} X\right)=0 \quad \text { for } i>0 .
$$


By the induction assumption we only need to consider $i=1$. So we want to prove that $\operatorname{Hom}\left(J, \tilde{\tau}^{-t+1} X\right)=0$ for $J$ indecomposable in $\mathcal{I}$, and we do this by induction on the length of $J$ which by assumption is finite. We have the AuslanderReiten triangle $J \stackrel{g}{\rightarrow} J / \operatorname{soc} J \oplus(\operatorname{rad} P)[1] \rightarrow P[1] \rightarrow J[1]$, with $P$ indecomposable projective in $\mathcal{P}$. Then any non-zero map $h: J \rightarrow \tilde{\tau}^{-t+1} X$ would factor through $g$ since $h$ is not an isomorphism. Note that by the induction assumption we have $\operatorname{Hom}\left((\operatorname{rad} P)[1], \tilde{\tau}^{-t+1} X\right)=\operatorname{Ext}^{-1}\left(\operatorname{rad} P, \tilde{\tau}^{-t+1} X\right)=0$, so the claim follows.

Case 3. $A$ falls under (C2) and $B$ falls under (C1). Thus $A=\tilde{\tau}^{-t} X$ for some $X$ in $\mathcal{B}$ and $t>0$. We have $\operatorname{Ext}^{-i}(A, B)=\operatorname{Ext}^{-i}\left(X, \tilde{\tau}^{t} B\right)$. Since $\tilde{\tau}^{t} B \in \mathcal{B}[l]$ for $l \leq 0$, this case is trivial.

Case 4. $A$ and $B$ both fall under (C2). This case can be reduced to one of the previous cases by applying powers of $\tilde{\tau}$.

So $\mathcal{C}$ is indeed an abelian category. Since it is easily seen that $F(\mathcal{C}) \subset \mathcal{C} \oplus \mathcal{C}[1]$, we obtain by Serre duality that $\operatorname{Hom}(\mathcal{C}, \mathcal{C}[n])=0$ for $n \neq 0,1$. Hence it follows by Lemma 1.3 .5 that $\mathcal{C}$ is hereditary.

Now we verify properties $1 .-4$.

1. This is clear from the construction.

3. This follows from Lemma I.3.5.

4. This is clear from the construction.

2. By 3. we already know that $\mathcal{C}$ has a Serre functor, so we can use its properties. An indecomposable object $P$ in $\mathcal{C}$ is projective precisely when $F P \in \mathcal{C}$. Using the construction of $\mathcal{C}$ it is easy to see that this happens if and only if $P \in \mathcal{P}$. Using the properties of Serre functors we find that the injectives in $\mathcal{C}$ must be given by $F(\mathcal{P})=\mathcal{I}$. This proves what we want.

If $Q$ is a quiver satisfying $(\mathrm{P} 1),(\mathrm{P} 2)$ and $\mathcal{B}=\operatorname{rep}(Q)$, then in the sequel we will denote by $\widetilde{\operatorname{rep}}(Q)$ the hereditary abelian category $\mathcal{C}$ whose existence is asserted by Theorem I.1.3

Example II.1.4. Consider the example

$$
0 \rightarrow 1 \rightarrow 2 \rightarrow \cdots
$$

Since the category $\operatorname{rep}(Q)$ has right almost split sequences, it makes sense to talk about an associated right AR-quiver, which looks like:

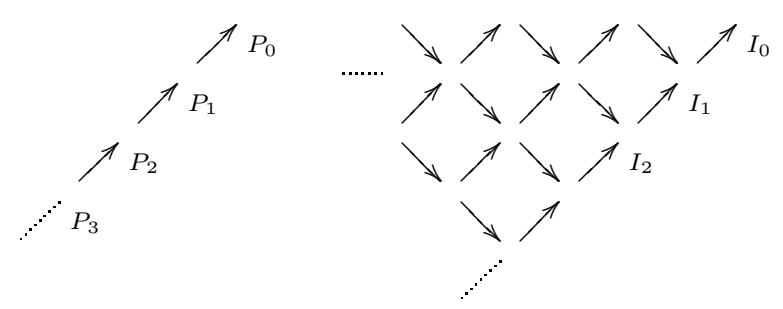


The AR-quiver for the new category $\widetilde{\operatorname{rep}}(Q)$ consists of the following two components:

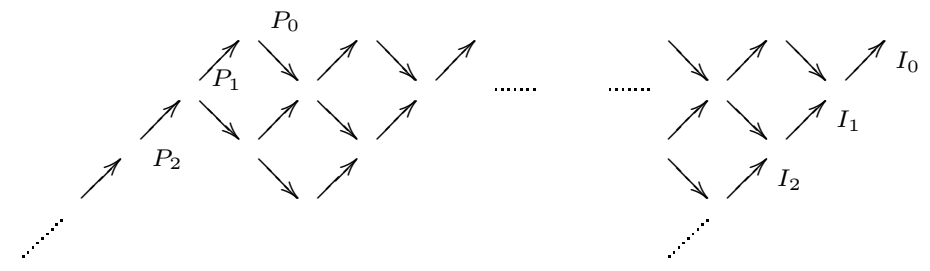

The new objects are the $\tau^{-i} P_{j}$ for $j \geq 0$ and $i>0$.

Remark II.1.5. It is not necessarily true that all injectives in $\operatorname{rep}(Q)$ are in $\mathcal{I}$. E.g. in the above example the projective representation associated to 0 is also injective. However it is not in $\mathcal{I}$.

II.2. Hereditary abelian categories generated by preprojectives. Let $\mathcal{C}$ be an Ext-finite hereditary abelian category. In this section we associate a quiver $Q$ with $\mathcal{C}$. We show that if $\mathcal{C}$ has Serre duality, the injective objects in $\mathcal{C}$ are noetherian and $\mathcal{C}$ is generated by the preprojective objects, then $\mathcal{C}$ is equivalent to $\widetilde{\text { rep }}(Q)$.

For an Ext-finite hereditary abelian category $\mathcal{C}$, denote as before by $\mathcal{P}$ and $\mathcal{I}$ respectively the full subcategories of $\mathcal{C}$ consisting of projective and injective objects. By $\overline{\mathcal{P}}$ we denote the full subcategory of $\mathcal{C}$ whose objects are quotients of objects in $\mathcal{P}$. For later reference we state the following easily proved fact.

Lemma II.2.1. $\overline{\mathcal{P}}$ is closed under subobjects and extensions. In particular $\overline{\mathcal{P}}$ is an abelian category.

To $\mathcal{C}$ we associate a quiver $Q$ whose vertices are in one-one correspondence with the indecomposable projectives in $\mathcal{C}$. If $x$ is a vertex, then we denote the corresponding projective object in $\mathcal{C}$ by $\mathfrak{p}_{x}$. For $x$ and $y$ vertices in $Q$ we let the arrows from $x$ to $y$ index elements $\left(f_{y, x}^{i}\right)_{i} \in \operatorname{rad} \operatorname{Hom}\left(\mathfrak{p}_{y}, \mathfrak{p}_{x}\right)$, which are representatives for a basis of $\operatorname{rad} \operatorname{Hom}\left(\mathfrak{p}_{y}, \mathfrak{p}_{x}\right) / \operatorname{rad}^{2} \operatorname{Hom}\left(\mathfrak{p}_{y}, \mathfrak{p}_{x}\right)$. Somewhat inaccurately we will say below that the projectives in $\mathcal{C}$ are given by $Q$. We now have a functor

$$
i: \operatorname{rep}(Q) \rightarrow \overline{\mathcal{P}}: P_{x} \mapsto \mathfrak{p}_{x}
$$

where $P_{x}$ is the projective representation of $Q$ associated with the vertex $x$. It is easy to see that this functor is faithful, but it is not necessarily full, as the following example shows.

Example II.2.2. Let $\operatorname{Gr}^{\dagger} k[x]$ be the category of graded $k[x]$-modules which are contained in a finite direct sum of indecomposable graded injective $k[x]$-modules, and let $\mathcal{C}=\left(\operatorname{Gr}^{\dagger} k[x]\right)^{\text {opp }}$. From the fact that $k[x]$ is hereditary one deduces that the same holds for $\mathcal{C}$. The indecomposable graded injective $k[x]$-modules are given by $E_{\infty}=k\left[x, x^{-1}\right]$ and $E_{a}=x^{a} k\left[x^{-1}\right]$ for $a \in \mathbb{Z}$. These correspond to projective objects $P_{a}$ in $\mathcal{C}$ for $a \in \mathbb{Z} \cup\{\infty\}$. We have $\operatorname{Hom}_{\mathcal{C}}\left(P_{a}, P_{\infty}\right)=\operatorname{Hom}_{\mathrm{Gr} k[x]}\left(E_{\infty}, E_{a}\right)=$ $k$. Thus $\operatorname{rad} \operatorname{Hom}_{\mathcal{C}}\left(P_{\infty}, P_{\infty}\right)=0$. Furthermore if $a \neq \infty$, then every map $P_{a} \rightarrow P_{\infty}$ factors through $P_{a+1}$. It follows that $\operatorname{rad} \operatorname{Hom}_{\mathcal{C}}\left(P_{a}, P_{\infty}\right)=\operatorname{rad}^{2} \operatorname{Hom}_{\mathcal{C}}\left(P_{a}, P_{\infty}\right)$ and thus $P_{\infty}$ is an isolated point in the quiver associated to $\mathcal{C}$. This contradicts fullness.

We now have the following result.

Proposition II.2.3. Assume that $\mathcal{C}$ has a Serre functor. Then the following are equivalent: 
1. $\mathcal{C}$ has finite length injectives.

2. $\mathcal{C}$ has noetherian injectives.

3. The quiver $Q$ satisfies (P1), (P2).

4. The quiver $Q$ satisfies $(\mathrm{P} 1),(\mathrm{P} 2)$, and $i: \operatorname{rep}(Q) \rightarrow \overline{\mathcal{P}}$ defines an equivalence between $\operatorname{rep}(Q)$ and $\overline{\mathcal{P}}$. In addition the indecomposable injectives in $\mathcal{C}$ are of the form $i\left(I_{x}\right)$ for $x \in Q$, so that in particular $\mathcal{I} \subset \overline{\mathcal{P}}$.

Proof.

1. $\Rightarrow 2$. This is clear.

$2 . \Rightarrow 1$. Assume that $I$ is an injective object in $\mathcal{C}$. By decomposing $I$ into a direct sum of indecomposables and invoking Theorem $[.3 .3$ we see that the socle $S$ of $I$ is non-zero and of finite length.

Since $\mathcal{C}$ is hereditary, $I / S$ is again injective. Repeating this we find a strictly ascending chain

$$
0=I_{0} \subset I_{1} \subset \cdots \subset I_{n} \subset \cdots \subset I
$$

such that $I_{n+1} / I_{n}$ has finite length. Since $I$ is noetherian, this chain must stop, whence $I$ has finite length.

1 . $\Rightarrow 4$. We first show that $Q$ satisfies (P2). In fact we show that for a vertex $z$ in $Q$ there is a bound on the length of a chain of non-isomorphisms

$$
\mathfrak{p}_{z} \rightarrow \mathfrak{p}_{z_{1}} \rightarrow \cdots \rightarrow \mathfrak{p}_{z_{n}}
$$

Put $\mathfrak{i}_{x}=F\left(\mathfrak{p}_{x}\right)$. Applying $F$ we get non-isomorphisms

$$
\mathfrak{i}_{z} \rightarrow \mathfrak{i}_{z_{1}} \rightarrow \cdots \rightarrow \mathfrak{i}_{z_{n}} .
$$

Since these are indecomposable injectives and $\mathcal{C}$ is hereditary, all these maps must be surjective. Clearly the required bound is now given by the length of $\mathfrak{i}_{z}$.

Now we prove that $Q$ satisfies $(\mathrm{P} 1)$. Since every $\operatorname{rad}\left(\mathfrak{p}_{x}\right)$ has only a finite number of summands in a direct sum decomposition, it is sufficient to show that there exists only a finite number of vertices $y$ such that there is a non-zero map $\mathfrak{p}_{x} \rightarrow \mathfrak{p}_{y}$. Applying $F$ we find that $\operatorname{soc}\left(\mathfrak{i}_{y}\right)$ must be a subquotient of $\mathfrak{i}_{x}$. Since $\mathfrak{i}_{x}$ has finite length, there is only a finite number of possibilities for $y$.

The fact that there is a bound (depending only on $z$ ) on chains of the form (II.2.1 implies that every map $\mathfrak{p}_{z} \rightarrow \mathfrak{p}_{t}$ is a linear combination of products of the $f_{y, x}^{i}$. Thus $i$ is full, and from this we easily obtain that $i$ yields an equivalence between $\operatorname{rep}(Q)$ and $\overline{\mathcal{P}}$.

Now let $I$ be indecomposable injective in $\mathcal{C}$. Since $\mathcal{C}$ has a Serre functor, it follows from Theorem $[1.3 .3$ that $I$ is the injective hull of some simple object $S$ lying in $\overline{\mathcal{P}}$. By considering the injective $I / S$ and using induction on the length of $I$ we find that $I \in \overline{\mathcal{P}}$. Now $I$ is clearly the injective hull of $S$ also in $\overline{\mathcal{P}}$. Using the fact that $i$ is an equivalence there must exist a vertex $x \in Q$ such that $S=i\left(S_{x}\right)$. Furthermore $I$ must correspond under $i$ to the injective hull of $S_{x}$. Since the latter is $I_{x}$, we are done.

4. $\Rightarrow 3$. This is clear.

$3 . \Rightarrow 1$. This proof is similar to that of $2 . \Rightarrow 1$. Assume that $I$ is an indecomposable injective of infinite length, and let $S$ be its socle. There exists an indecomposable summand $I_{1}$ of $I / S$ which is of infinite length. Continuing this 
procedure we find an infinite sequence of irreducible maps

$$
I \rightarrow I_{1} \rightarrow I_{2} \rightarrow \cdots
$$

and applying $F^{-1}$ we find a corresponding infinite sequence of irreducible maps between projectives

$$
P \rightarrow P_{1} \rightarrow P_{2} \rightarrow \cdots
$$

However such an infinite sequence cannot exist by (P2).

We have the following result on reconstructing $\mathcal{C}$ from the associated quiver $Q$.

Corollary II.2.4. Assume that $\mathcal{C}$ is an Ext-finite hereditary abelian category possessing a Serre functor $F$. Let the projectives in $\mathcal{C}$ be given by the quiver $Q$. Assume that $\mathcal{C}$ satisfies any of the conditions of Proposition II.Q.3 and furthermore that every indecomposable in $\mathcal{C}$ is of the form $\tau^{-t} X$ with $X \in \overline{\mathcal{P}}$. Then $\mathcal{C}$ is equivalent to $\widetilde{\mathrm{rep}}(Q)$.

Proof. From the fact that $F(\mathcal{P})=\mathcal{I} \subset \overline{\mathcal{P}}$ and the fact that $D^{b}(\overline{\mathcal{P}})$ is closed under cones in $D^{b}(\mathcal{C})$, it follows that $D^{b}(\overline{\mathcal{P}})$ is closed under $F$. Thus $F$ defines a right Serre functor on $\overline{\mathcal{P}}$, and hence via $i: \operatorname{rep}(Q) \rightarrow \mathcal{C}$ a right Serre functor on $\operatorname{rep}(Q)$. Since right Serre functors are unique, $F$ coincides (up to a natural isomorphism) with the standard right Serre functor obtained from deriving the Nakayama functor which was introduced in (II.1.2).

It now follows that $\mathcal{C}$ satisfies properties 1.,2.,3.,4. of Theorem II.1.3. Hence we get $\mathcal{C}=\widetilde{\operatorname{rep}}(Q)$.

We remind the reader of some elementary facts concerning preprojective objects. In general if $\mathcal{C}$ is a hereditary abelian category with almost split sequences, then a preprojective object is by definition an object of the form $\bigoplus_{i=1}^{n} \tau^{-a_{i}} P_{i}$ where the $P_{i}$ 's are indecomposable projectives and $a_{i} \geq 0$. Similarly a preinjective object is an object of the form $\bigoplus_{i=1}^{n} \tau^{a_{i}} I_{i}$ where the $I_{i}$ are indecomposable injective and $a_{i} \geq 0$.

For simplicity we restrict ourselves here to the case where $\mathcal{C}$ is Ext-finite with a Serre functor. In this case we have the functor $\tau: \mathcal{C}_{\mathcal{P}} \rightarrow \mathcal{C}_{\mathcal{I}}$ inducing the correspondence between the end terms of an almost split sequence.

Note the following.

Lemma II.2.5. Let $U \in \mathcal{C}$ be indecomposable, and assume there is a non-zero map $\phi: U \rightarrow \tau^{-a} P$ where $P$ is an indecomposable projective and $a \geq 0$. Then $U$ is of the form $\tau^{-b} Q$ where $Q$ is an indecomposable projective and $b \leq a$.

Proof. If $U$ is projective, then we are done. If $a=0$, then the fact that $U$ is indecomposable plus the fact that $\mathcal{C}$ is hereditary implies that $U$ is projective. Hence this case is covered also. Assume now that $U$ is not projective (and hence $a \neq 0$ ). Then by faithfulness of $\tau$ we obtain a non-zero map $\tau U \rightarrow \tau^{-a+1} P$. Induction on $a$ now yields that $\tau U=\tau^{-c} Q$ with $c \leq a-1$ for some indecomposable projective $Q$. Thus $U=\tau^{-c-1} Q$ and we are done.

Corollary II.2.6. 1. Assume that we have a map $\phi: U \rightarrow Q$ where $Q$ is preprojective. Then $U=U^{\prime} \oplus U^{\prime \prime}$ where $U^{\prime} \subset \operatorname{ker} \phi$ and $U^{\prime \prime}$ is preprojective.

2. Every subobject of a preprojective object is preprojective. 
Proof. 2. follows trivially from 1 ., so we prove 1 . Let $U=U_{1} \oplus \cdots \oplus U_{m}$ be a decomposition of $U$ into a direct sum of indecomposable objects. If $U_{i} \not \subset \operatorname{ker} \phi$, then it maps non-trivially to $Q$, whence it is preprojective by the previous lemma. This proves what we want.

We say that $\mathcal{C}$ is generated by preprojectives if every object in $\mathcal{C}$ is a quotient of a preprojective object. One has the following result.

Lemma II.2.7. The following are equivalent for an Ext-finite hereditary abelian category with Serre functor:

1. $\mathcal{C}$ is generated by preprojectives.

2. Every indecomposable object in $\mathcal{C}$ is of the form $\tau^{-t} X$ with $X \in \overline{\mathcal{P}}$ and $t \geq 0$.

Proof. Let us first assume 2., and let $Y=\tau^{-t} X \in \mathcal{C}$, with $X \in \overline{\mathcal{P}}$ and $t \geq 0$. We have to show that $Y$ is the quotient of a preprojective object. Take a minimal projective presentation

$$
0 \rightarrow Q \rightarrow P \rightarrow X \rightarrow 0
$$

with $P, Q \in \mathcal{P}$. For $l<t$ the object $\tau^{-l} Q$ cannot contain an injective summand since then the resolution (II.2.2) wasn't minimal. It follows that the object $\tau^{-l} P$ cannot contain an injective summand since then $\tau^{-l} X$ would be injective, contradicting the fact that it is in the essential image of $\tau$. Hence $\tau^{-t} Q$ and $\tau^{-t} P$ are defined, and by the exactness of $F$ (see [8]) we obtain an exact sequence

$$
0 \rightarrow \tau^{-t} Q \rightarrow \tau^{-t} P \rightarrow \tau^{-t} X \rightarrow 0
$$

In particular $\tau^{-t} X$ is covered by a preprojective object.

Now assume 1. Let $Y$ be an indecomposable object in $\mathcal{C}$, and assume that there is a surjective map $\phi: R \rightarrow Y$ with $R$ preprojective. Then by Corollary $\amalg .2 .6$ we have that $S=\operatorname{ker} \phi$ is also preprojective. Thus for large $t$ we have a triangle

$$
F^{t} S \rightarrow F^{t} R \rightarrow F^{t} Y \rightarrow
$$

with $F^{t} R$ and $F^{t} S$ in the image of $D^{b}(\overline{\mathcal{P}})$. Since $D^{b}(\overline{\mathcal{P}})$ is closed under cones in $D^{b}(\mathcal{C})$ and since $F^{t} Y$ is indecomposable, it follows that $F^{t} Y \in \overline{\mathcal{P}}[l]$ for some $l$.

Thus if $\tau^{p} Y$ is defined for $1 \leq p \leq t$, then $\tau^{t} Y \in \overline{\mathcal{P}}$. If $\tau^{p} Y$ is not defined, then $\tau^{p-1} Y$ is projective and so lies in $\mathcal{P}$. Hence in this case we are done also.

Combining the last lemma with Corollary 1.2 .4 we obtain the following.

Corollary II.2.8. Assume that $\mathcal{C}$ is an Ext-finite hereditary abelian category possessing a Serre functor $F$. Let the projectives in $\mathcal{C}$ be given by the quiver $Q$. Assume that $\mathcal{C}$ is generated by preprojectives and satisfies any of the conditions of Proposition 11.2.3. Then $\mathcal{C}$ is equivalent to $\widetilde{\operatorname{rep}}(Q)$.

We will also need the following result.

Theorem II.2.9. Let $\mathcal{Q}$ be the full subcategory of $\mathcal{C}$ whose objects are quotients of preprojective objects. Then $\mathcal{Q}$ is closed under subquotients and extensions. If in addition $\mathcal{C}$ satisfies any of the conditions of Proposition II.2.3, then the Serre functor on $D^{b}(\mathcal{C})$ restricts to a Serre functor on $D^{b}(\mathcal{Q})$.

Proof. Closedness under quotients is clear. Let us now prove closedness under subobjects. Clearly it is sufficient to show that a subobject of a preprojective object is preprojective. But this is precisely Corollary $\amalg .2 .6$. 
Let us now prove closedness under extensions. So assume that we have an exact sequence

$$
0 \rightarrow A \rightarrow B \rightarrow C \rightarrow 0
$$

where $A, C \in \mathcal{Q}$. Since we already know that $\mathcal{Q}$ is closed under quotients, we may prove our result for pullbacks of (11.2.4). In particular we may assume that $C$ is preprojective. But then according to Corollary 11.2 .6 we have $B=A_{1} \oplus Q$ where $A_{1} \subset A$ and $Q$ is preprojective. Hence by what we know already we have $A_{1} \in \mathcal{Q}$. This proves what we want.

Assume now that $\mathcal{C}$ satisfies any of the conditions of Proposition II.2.3. We have to show that $D^{b}(\mathcal{Q})$ is closed under $F$ and $F^{-1}$ in $D^{b}(\mathcal{C})$. Since $D^{b}(\mathcal{Q})$ is closed under the formation of cones, it suffices to show that $F(P)$ and $F^{-1}(P)$ are in $D^{b}(\mathcal{Q})$ for a preprojective object $P$. The only case which isn't entirely obvious is that $F(P)$ is in $D^{b}(\mathcal{Q})$ when $P$ is projective, but this follows from Proposition II.2.3

For completeness let us include the following lemma.

Lemma II.2.10. Let $Q$ be a quiver satisfying (P1), (P2). Then $Q$ is connected if and only if $\widetilde{\operatorname{rep}}(Q)$ is connected.

Proof. Since clearly $\widetilde{\operatorname{rep}}\left(Q_{1} \amalg Q_{2}\right)=\widetilde{\operatorname{rep}}\left(Q_{1}\right) \oplus \widetilde{\operatorname{rep}}\left(Q_{2}\right)$ it suffices to prove the implication that $Q$ connected implies $\widetilde{\operatorname{rep}}(Q)$ connected. So assume that $\mathcal{C}=\widetilde{\operatorname{rep}}(Q)$ is a direct sum $\mathcal{C}=\mathcal{C}_{1} \oplus \mathcal{C}_{2}$ with $\mathcal{C}_{1}$ and $\mathcal{C}_{2}$ non-trivial. Let $\mathcal{P}_{i}$ be the category of projectives in $\mathcal{C}_{i}$ for $i=1,2$. Then clearly $\mathcal{P}=\mathcal{P}_{1} \oplus \mathcal{P}_{2}$. This yields a corresponding decomposition $Q=Q_{1} \amalg Q_{2}$. Since we had assumed that $Q$ is connected, it follows that for example $Q_{2}=0$.

By LemmaI.1.11 the Serre functor on $D^{b}(\mathcal{C})$ restricts to one on $D^{b}\left(\mathcal{C}_{1}\right)$ and one on $D^{b}\left(\mathcal{C}_{2}\right)$. It is now easy to see that the conditions for Corollary I.2.4 descend to $\mathcal{C}_{1}$ and $\mathcal{C}_{2}$. Thus $\mathcal{C}_{i}=\widetilde{\operatorname{rep}}\left(Q_{i}\right)$ and hence $\mathcal{C}_{2}=0$. This is a contradiction.

II.3. Derived equivalences. In this section we construct a derived equivalence between $\widetilde{\operatorname{rep}}(Q)$ and $\widetilde{\operatorname{rep}}\left(Q^{\prime}\right)$ for certain quivers $Q$ and $Q^{\prime}$ satisfying (P1), (P2). This result will be used in the proof of Theorem $\mathrm{C}$ At the same time we get an alternative method for the construction of $\widetilde{\operatorname{rep}} Q$ from $\operatorname{rep} Q$.

If $Q$ is a quiver, then $\mathbb{Z} Q$ denotes the quiver whose vertices are of the form $(n, a)$ with $n \in \mathbb{Z}$ and $a \in Q$ and whose arrows are of the form $(n, a) \rightarrow(n, b)$ and $(n, b) \rightarrow(n+1, a)$ for any arrow $a \rightarrow b$. Then $\mathbb{Z} Q$ is a translation quiver with translation given by $\tau(n, a)=(n-1, a)$. The following is easy to see.

Lemma II.3.1. Assume that $Q$ satisfies (P1), (P2). Let $x, y \in \mathbb{Z} Q$. Then the number of paths in $\mathbb{Z} Q$ from $x$ to $y$ is finite.

For a quiver $Q$ satisfying (P1), (P2), denote by $\mathbb{N} Q$ the part of $\mathbb{Z} Q$ given by the vertices $(n, a)$ where $n \geq 0$. Let $Q^{\prime} \subset \mathbb{Z} Q$ be a section of $\mathbb{Z} Q$. By this we mean the following: $Q^{\prime}$ contains exactly one vertex from each $\tau$-orbit of $\mathbb{Z} Q$ and if $x \in Q^{\prime}$ and there is an arrow $x \rightarrow y$ in $\mathbb{Z} Q$, then either the arrow $x \rightarrow y$ or the arrow $\tau y \rightarrow x$ is in $Q^{\prime}$, and if there is an arrow $z \rightarrow x$, then either the arrow $z \rightarrow x$ or the arrow $x \rightarrow \tau^{-1} z$ is in $Q^{\prime}$. It is then clear that if $Q^{\prime}$ is a section in $\mathbb{Z} Q$, then $\mathbb{Z} Q^{\prime}=\mathbb{Z} Q$ and $Q$ is a section in $\mathbb{Z} Q^{\prime}$. Our main result is that the categories $\widetilde{\operatorname{rep}}(Q)$ and $\widetilde{\operatorname{rep}}\left(Q^{\prime}\right)$ are derived equivalent when $Q^{\prime}$ is a section in $\mathbb{Z} Q$. This is well known when $Q$ is finite, in which case $\widetilde{\operatorname{rep}}(Q)=\operatorname{rep}(Q)$ and $\widetilde{\operatorname{rep}}\left(Q^{\prime}\right)=\operatorname{rep}\left(Q^{\prime}\right)$, and one can go from $Q$ to $Q^{\prime}$ by a finite sequence of reflections. 
Lemma II.3.2. Let $\mathcal{C}$ be an Ext-finite hereditary abelian category possessing a Serre functor $F$. Assume that the projectives in $\mathcal{C}$ are given by a quiver satisfying $(\mathrm{P} 1),(\mathrm{P} 2)$. Then the preinjective objects in $\mathcal{C}$ have finite length, and furthermore they are quotients of projectives.

Proof. Let $\mathcal{P}, \mathcal{I} \subset \mathcal{C}$ have their usual meaning. Since $F(\mathcal{P})=\mathcal{I}$, we easily obtain $F\left(D^{b}(\mathcal{P})\right)=D^{b}(\mathcal{I})$, and by Proposition $\llbracket .2 .3$ we have that the objects in $\mathcal{I}$ have finite length and furthermore $D^{b}(\mathcal{I}) \subset D^{b}(\mathcal{P})$. Thus in particular $F\left(D^{b}(\mathcal{I})\right) \subset$ $D^{b}(\mathcal{I})$. Iterating we find $F^{a} \mathcal{I} \subset D^{b}(\mathcal{I})$ for any $a>0$. This proves that the preinjective objects have finite length. We also obtain $F^{a} \mathcal{I} \subset D^{b}(\mathcal{P})$, which yields that the preinjective objects are quotients of projectives.

Also recall that a quiver $Q$ is Dynkin if the underlying graph is a Dynkin diagram, that is, of the form $A_{n}, D_{n}$ or $E_{6}, E_{7}, E_{8}$, where $n$ denotes the number of vertices. Note that $\operatorname{rep} Q$ is of finite representation type for a connected quiver $Q$ if and only if $Q$ is a Dynkin quiver.

We shall also need the infinite graphs $A_{\infty}, A_{\infty}^{\infty}$ and $D_{\infty}$ :

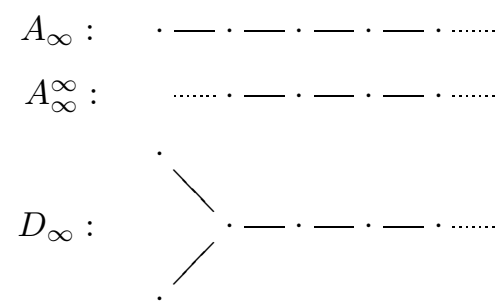

We also need the following. Here the preprojective component of the AR-quiver denotes the component containing the projective objects and the preinjective component denotes the component containing the injective objects.

Lemma II.3.3. Assume that $Q$ is a connected quiver which is not Dynkin but satisfies (P1), (P2), and let $\mathcal{C}$ be an Ext-finite hereditary abelian category with Serre functor, whose projectives are given by $Q$.

1. The preprojective component $\Pi$ of $\mathcal{C}$ contains no injective objects.

2. There is an isomorphism $\Pi \cong \mathbb{N} Q^{\text {opp }}$ of quivers, compatible with the translation.

Proof. For $x \in Q$ let $P_{x}$ and $I_{x}$ be the corresponding projective and injective object in $\mathcal{C}$. It is well known and easy to see that sending $\tau^{-n} P_{x}$ to $(n, x)$ defines an injective morphism of quivers $\Pi \rightarrow \mathbb{N} Q^{\text {opp }}$, where $\Pi$ is the preprojective component of $\mathcal{C}$. It is clear that this is surjective if and only if $\Pi$ contains no injectives. So it is sufficient to show that $\Pi$ contains no injective objects.

Assume to the contrary that $\Pi$ has some injective object. The projectives are given by the connected quiver $Q$, and the arrows of $Q$ correspond to irreducible maps between indecomposable projectives, in the opposite direction. Because the categories of injectives and projectives are equivalent, the same holds for the injectives. Thus $\Pi$ contains all indecomposable injectives. In particular $\Pi$ is also the preinjective component of $\mathcal{C}$, and hence by Lemma $\amalg .3 .2$ all objects in $\Pi$ have finite length.

Assume that there is some finite connected subquiver $Q_{1}$ of $Q$ which is not Dynkin. Choose $x \in Q_{1}$ and let $Q_{2} \supset Q_{1}$ be a finite connected subquiver of $Q$ such that the simple composition factors of all objects in any path from $P_{x}$ to $I_{x}$ 
correspond to vertices in $Q_{2}$. In the subcategory $\operatorname{rep}\left(Q_{2}\right)$ of $\mathcal{C}$ we clearly have that $P_{x}$ and $I_{x}$ are still projective and injective objects respectively, and it is not hard to see that there is still a path of irreducible maps from $P_{x}$ to $I_{x}$. But since $Q_{2}$ is not Dynkin, it is well known that the preprojective component of $\operatorname{rep} Q_{2}$ has no injective objects, and we have a contradiction.

If all finite subquivers of $Q$ are Dynkin, then it is easy to see directly that $Q$ must be of type $A_{\infty}, A_{\infty}^{\infty}$ or $D_{\infty}$. These cases can be taken care of by direct computations (see III.3).

In order to prove the main result in this section we shall use the principle of tilting with respect to torsion pairs 16 .

We recall the main features in the special case we need. If $\mathcal{H}$ is a hereditary abelian category, then a split torsion $\operatorname{pair}(\mathcal{T}, \mathcal{F})$ is a pair of additive subcategories of $\mathcal{H}$ satisfying $\operatorname{Hom}(\mathcal{T}, \mathcal{F})=\operatorname{Ext}^{1}(\mathcal{F}, \mathcal{T})=0$, and having in addition the property that for every $A \in \mathcal{H}$ there exist $T \in \mathcal{T}$ and $F \in \mathcal{F}$ such that $A \cong T \oplus F$.

The theory of $t$-structures $\left[7\right.$ is used to obtain inside $D^{b}(\mathcal{H})$ a hereditary abelian subcategory $\mathcal{H}^{\prime}$ with split torsion pair $(\mathcal{F}[1], \mathcal{T})$. This construction of $\mathcal{H}^{\prime}$ from $\mathcal{H}$ is called tilting with respect to the torsion pair $(\mathcal{T}, \mathcal{F})$ (see [16]). Furthermore in this case we have $D^{b}(\mathcal{H}) \cong D^{b}\left(\mathcal{H}^{\prime}\right)$. This can be seen by combining 6, Appendix] (which defines a functor $D^{b}(\mathcal{H}) \rightarrow D^{b}\left(\mathcal{H}^{\prime}\right)$ ) with [16, p. 13] (which shows that this functor is fully faithful).

It is easily seen that if $\mathcal{H}$ is a Krull-Schmidt category, then in order to specify $(\mathcal{T}, \mathcal{F})$ it is necessary and sufficient to give a partition of the indecomposable objects in $\mathcal{H}:\left(T_{i}\right)_{i \in I},\left(F_{j}\right)_{j \in J}$ with properties $\operatorname{Hom}\left(T_{i}, F_{j}\right)=\operatorname{Ext}^{1}\left(F_{j}, T_{i}\right)=0$. Then $\mathcal{T}$ and $\mathcal{F}$ are respectively the additive categories with indecomposable objects $\left(T_{i}\right)_{i}$ and $\left(F_{j}\right)_{j}$. Note that in the presence of Serre duality the condition $\operatorname{Ext}^{1}\left(F_{j}, T_{i}\right)=0$ is often automatic.

We now identify appropriate split torsion pairs in our hereditary abelian categories.

Lemma II.3.4. Let $\mathcal{C}$ be a hereditary abelian Ext-finite category with a Serre functor whose projectives are given by a connected quiver $Q$ which is not Dynkin but satisfies (P1), (P2). Let $Q^{\prime} \subset \mathbb{Z} Q$ be a section.

1. Let $\mathcal{T}$ be the additive category generated by the objects in the preinjective component, and let $\mathcal{F}$ be the additive category whose indecomposable objects are not preinjective. Then $(\mathcal{T}, \mathcal{F})$ is a split torsion pair in $\mathcal{C}$.

2. Let $\mathcal{C}_{1}$ denote the hereditary abelian subcategory of $D^{b}(\mathcal{C})$ obtained by tilting with respect to $(\mathcal{T}, \mathcal{F})$ and shifting one place to the right (thus somewhat informally: $\left.\mathcal{C}_{1}=\mathcal{T}[-1] \oplus \mathcal{F}\right)$. The category $\mathcal{C}_{1}$ has a component $\Sigma$ of type $\mathbb{Z} Q^{\text {opp }}$ put together from the preprojective and preinjective component in $\mathcal{C}$ (see Figure II.3.2). In particular $\mathcal{C}_{1}$ is derived equivalent to $\mathcal{C}$ and has no non-zero projectives or injectives.

Consider now the section $Q^{\prime}$ in $\mathbb{Z} Q$ (see Figure II.3.3). Let $\mathcal{F}_{1}$ be the additive category generated by the indecomposable objects of the form $\tau^{i} C$ for $C \in Q^{\prime}$ and $i>0$, and let $\mathcal{T}_{1}$ be the additive category generated by the other indecomposable objects in $\mathcal{C}_{1}$. Then $\left(\mathcal{T}_{1}, \mathcal{F}_{1}\right)$ is a split torsion pair in $\mathcal{C}_{1}$.

3. Let $\mathcal{D}$ be the hereditary abelian category obtained by tilting $\mathcal{C}_{1}$ with respect to $\left(\mathcal{T}_{1}, \mathcal{F}_{1}\right)$. Then $\mathcal{D}$ is derived equivalent to $\mathcal{C}$ and the projectives in $\mathcal{D}$ are given by $Q^{\prime}$. 
$\tau$

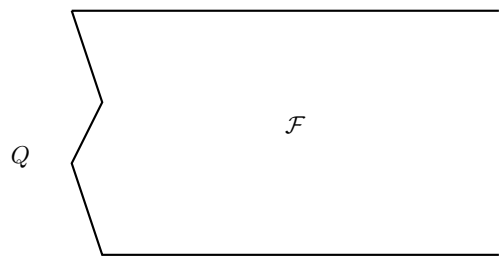

Preprojectives

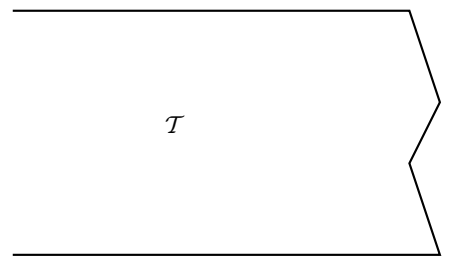

Preinjectives

$\mathcal{F}$

Other components

Figure II.3.1. The AR-quiver of $\mathcal{C}$

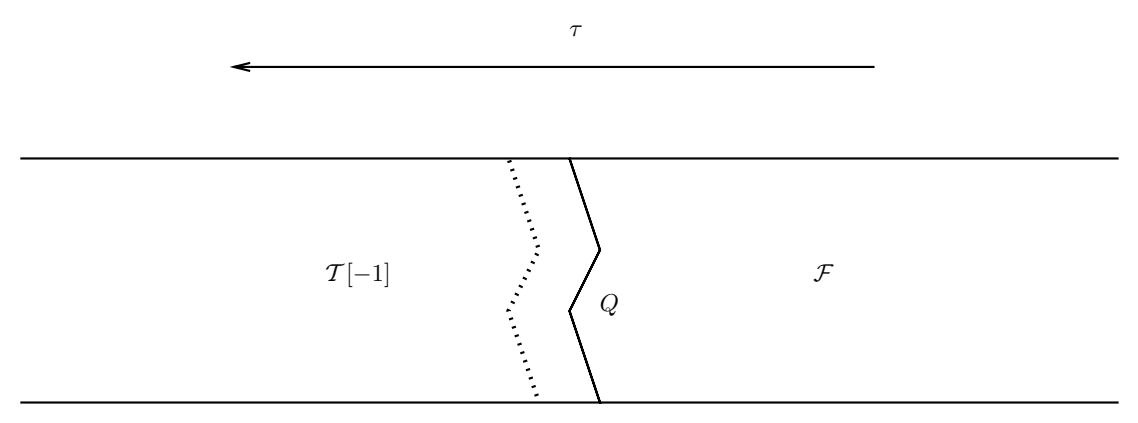

Joined components $(\Sigma)$

$\mathcal{F}$

Other components

Figure II.3.2. The AR-quiver of $\mathcal{C}_{1}$ 


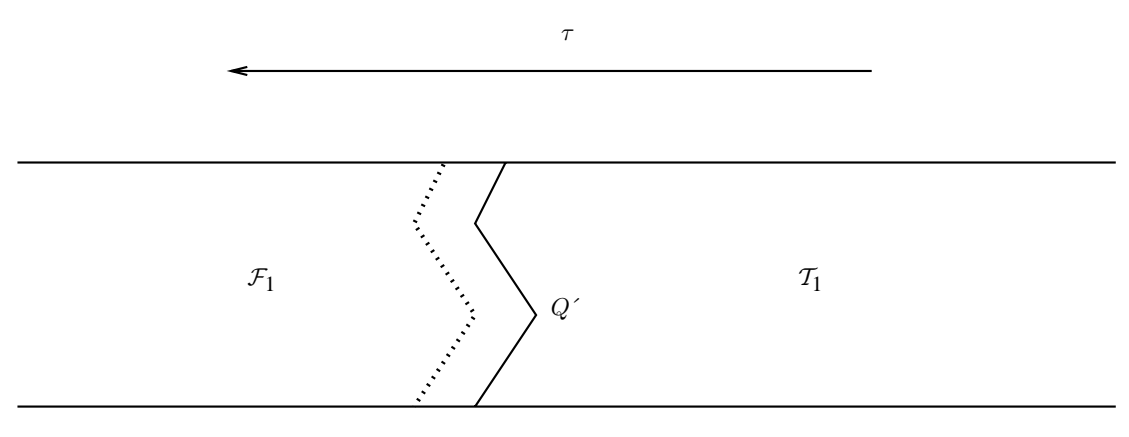

Joined components $(\Sigma)$

Other components

FiguRE II.3.3. A new torsion pair in $\mathcal{C}_{1}$

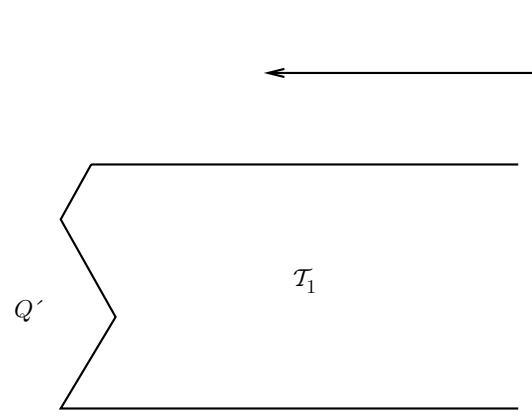

Preprojectives

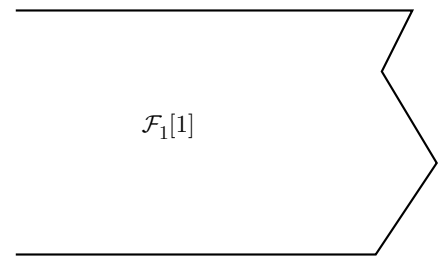

Preinjectives

$\mathcal{T}_{1}$

Other components

Figure II.3.4. The AR-quiver of $\mathcal{D}$ 
Proof. The proof amounts to verifying the conditions for a split torsion pair. In order to visualize this we have included some pictures of the various AR-quivers involved. Let the functor $\tau$ be defined as usual.

1. By the dual version of Lemma 1.2 .5 we find $\operatorname{Hom}(\mathcal{T}, \mathcal{F})=0$. If $T$ is a noninjective indecomposable object in $\mathcal{T}$, then we have that $\tau^{-1} T \in \mathcal{T}$. Hence it follows by Serre duality that $\operatorname{Ext}^{1}(\mathcal{F}, \mathcal{T})=0$. Thus $(\mathcal{T}, \mathcal{F})$ is a split torsion pair in $\mathcal{C}$.

2. Now we need to show $\operatorname{Hom}\left(\mathcal{T}_{1}, \mathcal{F}_{1}\right)=0$.

First let $B$ be indecomposable in $\Sigma$, and let $A$ be an indecomposable which is not in $\Sigma$ (and hence in particular $A \in \mathcal{T}_{1}$ ). Then looking at Figure II.3.2 we see that either $B \in \mathcal{T}[-1]$ or $B \in \mathcal{F}$. In the first case it is clear that $\operatorname{Hom}(A, B)=0$ and in the second case this follows from the fact that $(\mathcal{T}, \mathcal{F})$ is a split torsion pair.

Now let $A$ and $B$ be in $\Sigma$. By applying $\tau^{-n}$ for $n$ large enough so that $\tau^{-n} A$ and $\tau^{-n} B$ are in the preprojective component of $\mathcal{C}$, it is not hard to see that if $\operatorname{Hom}(A, B) \neq 0$, then there is a path from $A$ to $B$ in the AR-quiver of $\mathcal{C}_{1}$. If $\operatorname{Hom}(A, B) \neq 0$ for some $A \in \mathcal{T}_{1}$ and $B \in \mathcal{F}_{1}$, we can assume that there is an arrow from $A$ to $B$. Write $A=\tau^{i} X$ and $B=\tau^{j} Y$ where $X$ and $Y$ are in $Q^{\prime}$ and $i \leq 0$ and $j>0$. Then we have an arrow $\tau^{-i} A=X \rightarrow \tau^{j-i} Y$. Since $Q^{\prime}$ is a section in $\mathbb{Z} Q$ we have $\tau^{j-i} Y \in Q^{\prime}$ or $\tau^{j-i+1} Y \in Q^{\prime}$. Since $Y \in Q^{\prime}$ we conclude that $j=i$ or $j=i-1$, so $j \leq 0$ which is a contradiction.

Since $\mathcal{T}_{1}$ is $\tau^{-1}$ stable, the fact that $\operatorname{Ext}^{1}\left(\mathcal{F}_{1}, \mathcal{T}_{1}\right)=0$ follows from Serre duality.

3. That the projectives in $\mathcal{D}$ are given by $Q^{\prime}$ follows from the shape of the ARquiver of $\mathcal{D}$.

We shall also need the following.

Proposition II.3.5. Let $Q$ and $Q^{\prime}$ be non-Dynkin connected quivers satisfying $(\mathrm{P} 1),(\mathrm{P} 2)$, and assume that $Q^{\prime}$ is a section in $\mathbb{Z} Q$. Let $\mathcal{C}$ be a hereditary Ext-finite abelian category with a Serre functor whose projectives are given by the quiver $Q$, and assume that $\mathcal{C}$ is generated by preprojectives. Then the category $\mathcal{D}$ constructed in Lemma 11.3 .4 is also generated by preprojectives.

Proof. Let $C$ be an indecomposable object in $\mathcal{D}$. We have to show that it is a quotient of a preprojective object. If $C$ is itself preprojective, this is clear, and if $C$ is preinjective, then we can invoke Lemma II.3.2.

So assume that $C$ is neither in the preinjective nor preprojective component. Looking at Figures II.3.1 II.3.4 we see that $C$ is untouched by the tilting, so we may consider $C$ as an object in $\mathcal{C}$. Then by assumption there is an exact sequence in $\mathcal{C}$ of the form

$$
0 \rightarrow K \rightarrow P \rightarrow C \rightarrow 0
$$

where $P$, and consequently $K$, is preprojective. Since all terms in the exact sequence lie in the tilted category $\mathcal{C}_{1}$, this is also an exact sequence in $\mathcal{C}_{1}$. Let the functor $\tau$ be defined as usual. Choose $n>0$ large enough such that $\tau^{-n} K$ and $\tau^{-n} P$ are preprojective in $\mathcal{D}$, and consider the exact sequence

$$
0 \rightarrow \tau^{-n} K \rightarrow \tau^{-n} P \rightarrow \tau^{-n} C \rightarrow 0 .
$$


Thus $\tau^{-n} C$ is in the subcategory $\mathcal{Q}$ of $\mathcal{D}$ generated by the preprojectives. Since the Serre functor on $\mathcal{D}$ restricts to one on $\mathcal{Q}$ by Theorem $\amalg$ II.2.9 it follows that $C$ is also in $D^{b}(\mathcal{Q})$ and hence in $\mathcal{Q}$.

We now obtain the main result of this section.

Theorem II.3.6. Let $Q$ and $Q^{\prime}$ be connected quivers satisfying (P1), (P2), and assume that $Q^{\prime}$ is a section in $\mathbb{Z} Q$. Then the categories $\widetilde{\operatorname{rep}}(Q)$ and $\widetilde{\operatorname{rep}}\left(Q^{\prime}\right)$ are derived equivalent.

Proof. If $Q$ and $Q^{\prime}$ are Dynkin, this is well known, so we assume that $Q, Q^{\prime}$ are non-Dynkin.

It follows from Lemma $\amalg .2 .7$ and Theorem $\llbracket .1 .3$ that $\widetilde{\text { rep }}(Q)$ is generated by preprojectives. By Proposition II.3.5 the category $\mathcal{D}$ whose projectives are given by $Q^{\prime}$, and which is obtained by a sequence of the two tilts described above, also has the property that it is generated by preprojectives. Then it follows from Theorem II.1.3 that $\mathcal{D}$ is equivalent to $\widetilde{\mathrm{rep}}\left(Q^{\prime}\right)$. Since we already know that $\widetilde{\mathrm{rep}}(Q)$ and $\mathcal{D}$ are derived equivalent, this finishes the proof.

The results in this section can be used to give an alternative approach to the construction of the category $\widetilde{\mathrm{rep}}(Q)$ associated with the quiver $Q$ satisfying (P1), (P2), under the following assumption:

$\left.{ }^{*}\right)$ There is a section $Q^{\prime} \subset \mathbb{Z} Q$ with orientation such that there are no infinite paths in $Q^{\prime}$.

Then we have $\widetilde{\operatorname{rep}}\left(Q^{\prime}\right)=\operatorname{rep}\left(Q^{\prime}\right)$. The category $\mathcal{D}$ obtained by two tilts is also generated by preprojectives (we also have that $Q \subset \mathbb{Z} Q^{\prime}$ is a section) by Proposition [1.3.5. and hence is uniquely determined by $Q$.

We have no example where $(*)$ is not satisfied by a quiver $Q$ satisfying (P1), (P2), hence it is possible that the construction of $\widetilde{\mathrm{rep}}(Q)$ given in this section has the same generality as the one given in Theorem 11.1.3. In any case, as follows from the results in the next section, it is general enough for the classification in the noetherian case.

II.4. The classification. In this section we show that a connected noetherian hereditary abelian Ext-finite category with Serre functor and non-zero projective objects is generated by the preprojective objects, and hence is of the form $\widetilde{\operatorname{rep}}(Q)$ for some quiver $Q$. Then we describe the quivers $Q$ such that $\widetilde{\operatorname{rep}}(Q)$ is noetherian, completing our classification when there are non-zero projective objects.

Our first step is a decomposition theorem. We start with $\mathcal{C}$ not necessarily connected. As before let $\mathcal{Q}$ be the subcategory of $\mathcal{C}$ generated by the preprojectives. Let $\mathcal{R}$ be the full subcategory of $\mathcal{C}$ consisting of objects $R$ such that $\operatorname{Hom}(Q, R)=0$ for any preprojective $Q$ (or equivalently for any $Q \in \mathcal{Q}$ ).

Lemma II.4.1. $\mathcal{R}$ is closed under subquotients and extensions.

Proof. The only thing that is not entirely trivial is the fact that $\mathcal{R}$ is closed under quotients. So let $\alpha: R \rightarrow S$ be a surjective map in $\mathcal{C}$ with $R \in \mathcal{R}$. Let $\theta: Q \rightarrow S$ be a non-trivial map with $Q$ preprojective. Finally let

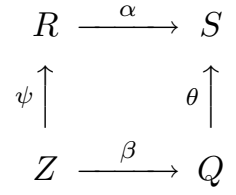


be the corresponding pullback diagram. Since $\theta \beta$ is not the zero map, there must exist an indecomposable summand $Z_{1}$ of $Z$ such that $\theta \beta\left(Z_{1}\right) \neq 0$. Since $Z_{1}$ maps non-trivially to $Q$, it follows from Corollary $\amalg .2 .6$ that it must be preprojective. Now clearly $\psi\left(Z_{1}\right) \neq 0$. This yields a contradiction.

Now we come to our main result in this section. Let $\mathcal{C}$ be an Ext-finite noetherian hereditary abelian category, and let the notation be as above.

Theorem II.4.2. $\quad$ 1. The inclusions $\mathcal{R} \rightarrow \mathcal{C}$ and $\mathcal{Q} \rightarrow \mathcal{C}$ define an equivalence $\mathcal{R} \oplus \mathcal{Q} \cong \mathcal{C}$.

2. The hereditary abelian categories $\mathcal{Q}$ and $\mathcal{R}$ satisfy Serre duality. If the functor $\tau$ is defined as usual, then $\tau$ is everywhere defined on $\mathcal{R}$ and is invertible.

Proof. 1. We have by definition $\operatorname{Hom}(\mathcal{Q}, \mathcal{R})=0$. Furthermore it follows from Lemma II.4.1 and Theorem $\amalg .2 .9$ that $\operatorname{Hom}(\mathcal{R}, \mathcal{Q})=0$. Hence it is sufficient to show that every $C \in \mathcal{C}$ is a direct sum $R \oplus Q$ with $R \in \mathcal{R}$ and $Q \in \mathcal{Q}$.

From the fact that $\mathcal{C}$ is noetherian and $\mathcal{Q}$ is closed under extensions (Theorem II.2.9 it follows that there exists a maximal subobject $Q$ in $C$ which lies in $\mathcal{Q}$, and so $R=C / Q$ lies in $\mathcal{R}$. Hence it now suffices to prove that $\operatorname{Ext}^{1}(R, Q)=0$. Since $\mathcal{C}$ is hereditary, $\operatorname{Ext}^{1}(R,-)$ preserves epis, thus it is sufficient to show that $\operatorname{Ext}^{1}\left(R, \tau^{-a} P\right)=0$ with $P$ an indecomposable projective and $a \geq 0$. In addition we may and we will assume that $R$ is indecomposable.

Assume $\operatorname{Ext}^{1}\left(R, \tau^{-a} P\right) \neq 0$. Then clearly $R$ is not projective and $\tau^{-a} P$ is not injective. Hence by Serre duality $\operatorname{Hom}\left(\tau^{-a-1} P, R\right) \neq 0$. This contradicts the fact that $R \in \mathcal{R}$. Hence $\operatorname{Ext}^{1}(R, Q)=0$, which finishes the proof.

2. That $\mathcal{Q}$ and $\mathcal{R}$ satisfy Serre duality follows for example from Lemma I.1.11 Any projective or injective object in $\mathcal{R}$ clearly has the same property in $\mathcal{C}=$ $\mathcal{R} \oplus \mathcal{Q}$ and therefore lies in $\mathcal{Q}$. Since a non-zero object cannot be both in $\mathcal{R}$ and $\mathcal{Q}$, we conclude that $\mathcal{R}$ does not contain non-zero projectives or injectives. Hence $\tau$ is defined everywhere on $\mathcal{R}$ and is invertible.

For an example showing that this theorem fails for non-noetherian hereditary abelian categories (even when they have noetherian injectives), see $₫$ II.3.6.

Theorem II.4.2 together with Corollary II.2.8 tells us that $\mathcal{C}$ is of the form $\widetilde{\operatorname{rep}}(Q) \oplus \mathcal{R}$ where $\mathcal{R}$ has a Serre functor. When $\mathcal{C}$ is connected, it follows that $\mathcal{C}=\widetilde{\operatorname{rep}}(Q)$. It remains to describe the $\widetilde{\mathrm{rep}}(Q)$ which are noetherian.

Let us first define a particular kind of quivers. We call a ray an infinite quiver of the form

$$
x: \quad x_{0} \rightarrow x_{1} \rightarrow x_{2} \rightarrow \cdots .
$$

We call $x_{0}$ the starting vertex.

We call a quiver $Q$ strongly locally finite if it is connected and if for every vertex in $Q$ the associated projective and injective representations have finite length. Note that a strongly locally finite quiver automatically satisfies (P1), (P2).

If $Q$ is a quiver, then by attaching a ray we mean identifying a vertex of $Q$ with the starting vertex of the ray.

Finally we call a quiver a star if it consists of a strongly locally finite quiver $Q_{0}$ to which a set of rays is simultaneously attached in such a way that only a finite number of rays are attached to every vertex. Note that a star is connected and satisfies (P1), (P2). 
We now prove the following result.

Theorem II.4.3. Let $Q$ be a connected quiver satisfying the properties (P1), (P2). Then $\widetilde{\operatorname{rep}}(Q)$ is noetherian if and only if $Q$ is a star.

The proof will consist of a number of lemmas.

We will be analyzing infinite paths in $Q$. These are by definition subquivers of $Q$ of the form

$$
x: \quad x_{0} \rightarrow x_{1} \rightarrow x_{2} \rightarrow \cdots
$$

(so actually infinite paths are the same as rays but we use different terminology to avoid confusion).

Two infinite paths are said to be equivalent if they are equal from some vertex on.

Lemma II.4.4. Assume that $\operatorname{rep}(Q)$ is noetherian. If $x$ is an infinite path in $Q$, then there exists $n_{0}$ such that for $n \geq n_{0}$ the only arrow in $Q$ starting in $x_{n}$ is the arrow in $x$ going from $x_{n}$ to $x_{n+1}$.

Proof. Assume that $n_{0}$ does not exist. We will show that then $\operatorname{rep}(Q)$ is not noetherian. The non-existence of $n_{0}$ implies that there exists a proper strictly ascending array of integers $\left(n_{i}\right)_{i \geq 1}$ as well as a corresponding array of vertices $\left(y_{i}\right)_{i}$ such that there is an arrow $e_{i}: x_{n_{i}} \rightarrow y_{i}$ which is not in $x$. The edges $e_{i}$ induce maps $\phi_{i}: P_{y_{i}} \rightarrow P_{x_{0}}$ which are the composition of the maps $P_{y_{i}} \stackrel{e_{i}}{\rightarrow} P_{x_{n_{i}}} \rightarrow P_{x_{0}}$.

We now claim that the image of $\left(\phi_{i}\right)_{i \leq m}: \bigoplus_{i \leq m} P_{y_{i}} \rightarrow P_{x_{0}}$ defines a proper ascending chain of subobjects of $P_{x_{0}}$. This is clear since the path in $Q$ corresponding to the map $\phi_{m+1}$ in $Q$ does not pass through $e_{i}$ for $i \leq m$. Hence $\operatorname{rep}(Q)$ is not noetherian, and we have obtained a contradiction.

In order to give a more precise analysis when $\widetilde{\operatorname{rep}}(Q)$ is noetherian we need the following.

Lemma II.4.5. Let $\mathcal{C}$ be a $k$-linear Ext-finite hereditary abelian category with a Serre functor $F$. Let $\tau$ be defined as usual.

Let $Q, P_{1}$ and $P_{2}$ be non-injective indecomposable projectives in $\mathcal{C}$. Assume we have an irreducible map $\alpha: Q \rightarrow \tau^{-1} P_{1}$ and a map $\beta: P_{1} \rightarrow P_{2}$ which does not factor through $Q$. Let $\varphi$ be the composition of maps

$$
Q \stackrel{\alpha}{\longrightarrow} \tau^{-1} P_{1} \stackrel{\tau^{-1} \beta}{\longrightarrow} \tau^{-1} P_{2} .
$$

Then for any factorization of $\phi$ through a projective object $X$

$$
Q \stackrel{\gamma}{\longrightarrow} X \stackrel{\psi}{\longrightarrow} \tau^{-1} P_{2}
$$

we have that $\gamma$ splits.

Proof. Assume that $\gamma$ is not split. Consider the almost split sequence

$$
0 \rightarrow Q \stackrel{\left(\delta_{i}\right)_{i}}{\longrightarrow} \bigoplus_{i=1, \ldots, m} Y_{i} \stackrel{\left(\epsilon_{i}\right)_{i}}{\longrightarrow} \tau^{-1} Q \rightarrow 0
$$

From the theory of almost split sequences it follows that we may take $\delta_{1}=\alpha$ and $Y_{1}=\tau^{-1} P_{1}$. Also by the theory of almost split sequences it follows that $\gamma=\sum_{i} \mu_{i} \delta_{i}$ for some maps $\mu_{i}: Y_{i} \rightarrow X$. Since $X$ is projective we have $\operatorname{Hom}\left(\tau^{-1} P_{1}, X\right)=0$, and hence $\mu_{1}=0$. Now consider the map $\theta: \bigoplus_{i} Y_{i} \rightarrow \tau^{-1} P_{2}$ given by

$$
\left(-\tau^{-1} \beta, \psi \mu_{2}, \ldots, \psi \mu_{m}\right) \text {. }
$$


Clearly $\sum \theta_{i} \delta_{i}=0$, whence there exists $\omega: \tau^{-1} Q \rightarrow \tau^{-1} P_{2}$ such that

$$
\begin{aligned}
-\tau^{-1} \beta & =\omega \epsilon_{1}, \\
\psi \mu_{i} & =\omega \epsilon_{i} \quad(i \geq 2) .
\end{aligned}
$$

Applying $\tau$ to the first of these equations yields a factorization of $\beta$, contradicting the hypotheses.

Lemma II.4.6. Assume that $\widetilde{\mathrm{rep}}(Q)$ is noetherian. If $x$ is an infinite path in $Q$, then there exists $n_{0}$ such that for $n \geq n_{0}$ the only arrows in $Q$ adjacent to $x_{n}$ are those in $x$.

Proof. If $\widetilde{\operatorname{rep}}(Q)$ is noetherian, then so is $\operatorname{rep}(Q)$ since the latter is a full subcategory closed under subobjects. Therefore it follows from the previous lemma that there is some $n_{0}^{\prime}$ such that for $n \geq n_{0}^{\prime}$ the only arrow starting in $x_{n}$ is the one which lies in $x$. By dropping some initial vertices in $x$ we may assume $n_{0}^{\prime}=0$.

Assume now that $n_{0}$ (as in the statement of the lemma) does not exist. Since injectives have finite length, it is clear that $P_{x_{0}}$ is not injective. We will show that $\tau^{-1} P_{x_{0}}$ is not noetherian in $\widetilde{\operatorname{rep}}(Q)$.

The non-existence of $n_{0}$ implies that there exists a strictly ascending array of integers $\left(n_{i}\right)_{i \geq 1}$ as well as a corresponding array of vertices $\left(y_{i}\right)_{i}$ such that there is an arrow $e_{i}: y_{i} \rightarrow x_{n_{i}}$ which is not in $x$.

The edges $e_{i}$ correspond to an irreducible map $P_{x_{n_{i}}} \rightarrow P_{y_{i}}$. Using the theory of almost split sequences there is a corresponding map $e_{i}^{\prime}: P_{y_{i}} \rightarrow \tau^{-1} P_{x_{n_{i}}}$. Also from the path $x$ we obtain a map $P_{x_{n_{i}}} \rightarrow P_{x_{0}}$ which gives rise to a corresponding map $\tau^{-1} P_{x_{n_{i}}} \rightarrow \tau^{-1} P_{x_{0}}$. We denote by $\phi_{i}$ the composition

$$
P_{y_{i}} \stackrel{e_{i}^{\prime}}{\longrightarrow} \tau^{-1} P_{x_{i}} \rightarrow \tau^{-1} P_{x_{0}} .
$$

We now claim that the image of $\left(\phi_{i}\right)_{i \leq m}: \bigoplus_{i \leq m} P_{y_{i}} \rightarrow \tau^{-1} P_{x_{0}}$ defines an ascending chain of subobjects of $\tau^{-1} P_{x_{0}}$. If this were not the case, then for some $m$ all $\phi_{m^{\prime}}$ and $m^{\prime}>m$ must factor through $\bigoplus_{i \leq m} P_{y_{i}}$. It follows that the resulting map $P_{y_{m^{\prime}}} \rightarrow \bigoplus_{i \leq m} P_{y_{i}}$ must be split since otherwise by Lemma 1 I.4.5 and (II.4.1) the map $P_{x_{m^{\prime}}} \rightarrow P_{x_{0}}$ factors through $P_{y_{m^{\prime}}}$, which is impossible given the construction of this map.

Thus it follows that $y_{m^{\prime}} \in\left\{y_{i} \mid i \leq m\right\}$. We conclude that some vertex $y$ occurs infinitely often among the $y_{i}$, but this contradicts condition (P1).

Thus we have shown that $\widetilde{\operatorname{rep}}(Q)$ is not noetherian, and in this way we have obtained a contradiction to the hypotheses.

Corollary II.4.7. Assume that $\widetilde{\mathrm{rep}}(Q)$ is noetherian. Then $Q$ is a star.

Proof. Let $\Omega$ be the set of equivalence classes of infinite paths in $Q$. For every $\omega \in \Omega$ we choose a representative $x_{\omega}$. By the previous lemma we can without loss of generality assume that the only edges in $Q$ adjacent to $x_{\omega, n}(n \geq 1)$ are those in $x_{\omega}$.

Now let $Q_{0}$ be obtained from $Q$ by removing for all $\omega \in \Omega$ the vertices $x_{\omega, n}$ for $n \geq 1$ as well as the edges in $x_{\omega}$. It is clear that $Q$ is obtained from $Q_{0}$ by adjoining the strings $\left(x_{\omega}\right)_{\omega \in \Omega}$. Furthermore $Q_{0}$ itself cannot contain any infinite paths since such an infinite path would have to be equivalent to one of the $x_{\omega}$. In particular it would have to contain vertices outside $Q_{0}$, which is of course a contradiction. It follows that $Q_{0}$ is strongly locally finite, and so $Q$ is indeed a star. 
The following lemma completes the proof of Theorem II.4.3

Lemma II.4.8. If $Q$ is a star, then $\widetilde{\operatorname{rep}}(Q)$ is noetherian.

Proof. Assume that $Q$ is a star. Since by construction $\widetilde{\operatorname{rep}}(Q)$ is generated by preprojectives, it suffices to show that the indecomposable preprojectives are noetherian. So we need to consider objects of the form $\tau^{-b} P_{x}$ with $x$ a vertex in $Q$ and $b \geq 0$.

Taking the sum of all irreducible maps (with multiplicities) going into $\tau^{-b} P_{x}$, we obtain a map $\bigoplus_{i} P_{i} \rightarrow \tau^{-b} P_{x}$, where the sum is finite, which is either surjective or has simple cokernel (the latter happens if $b=0$ ). In any case it is sufficient to show that the $P_{i}$ are noetherian.

By Lemma II.2.5 we know what the $P_{i}$ can be. They are of the form $\tau^{-c} P_{y}$ such that either $c<b$, or else $c=b$ and the map $\tau^{-b} P_{y} \rightarrow \tau^{-b} P_{x}$ is obtained from an irreducible map $P_{y} \rightarrow P_{x}$, which in turn corresponds to an arrow $x \rightarrow y$ in the quiver $Q$.

Induction on $b$ now yields that it is sufficient to show that $\tau^{-b} P_{z}$ is noetherian, where $z$ lies on one of the rays contained in $Q$, and furthermore for a given $b$ we may assume that $z$ lies arbitrarily far from the starting vertex of the ray.

Let $w$ be the ray on which $z$ lies. We will assume that $z=w_{b}$ (if $z$ happens to be farther away, then we just drop the initial vertices in $w$ ).

To check that $\tau^{-b} P_{z}$ is noetherian we need to understand the additive category $\mathcal{U}$ whose objects are direct sums of indecomposables which have a non-zero map to $\tau^{-b} P_{z}$. The theory of almost split sequence easily yields that $\mathcal{U}$ is the path category of the part of the AR-quiver of $\widetilde{\operatorname{rep}}(Q)$ spanned by the vertices that have a nontrivial path to $\tau^{-b} P_{z}$. Furthermore, also by the theory of almost split sequences, it is easy to work out what this quiver is. The result is as in Figure II.4.1. By convention we will assume that the relations on the quiver in Figure II.4.1 are of the form

$$
e_{v, u} f_{u, v}=f_{u+1, v} e_{v+1, u} .
$$

Note that from these relations, or directly, it easily follows that

$$
\operatorname{Hom}\left(\tau^{-c} P_{w_{m}}, \tau^{-c^{\prime}} P_{w_{m^{\prime}}}\right)= \begin{cases}k & \text { if } c \leq c^{\prime} \text { and } m \geq m^{\prime}, \\ 0 & \text { otherwise. }\end{cases}
$$

To show that $\tau^{-b} P_{w_{b}}$ is noetherian we have to show that there does not exist an infinite sequence of non-zero maps (unique up to a scalar by (II.4.3 ) $) \phi_{i}: \tau^{-c_{i}} P_{w_{n_{i}}} \rightarrow$ $\tau^{-b} P_{w_{b}}$, such that the image of $\phi_{m+1}$ is not contained in the image of $\bigoplus_{i \leq m} \phi_{i}$.

Assume to the contrary that such a sequence $\left(\phi_{i}\right)_{i}$ does indeed exist. Let $C \subset$ $\{0, \ldots, b\}$ be the set of all $c$ such that $c=c_{i}$ for some $i$. For every $c \in C$ let $v_{c}$ be the $w_{n_{i}}$ closest to $w_{0}$ with the property that $c=c_{i}$, and let $\theta_{c}$ be the corresponding $\phi_{i}$. It is now clear from the quiver given in Figure[I.4.1 as well as (II.4.3) that every $\phi_{i}$ factors through one of the $\theta_{c}$, contradicting the choice of $\left(\phi_{i}\right)_{i}$. This finishes the proof.

Summarizing, we have the following main result of this section.

Theorem II.4.9. The connected hereditary abelian noetherian Ext-finite categories $\mathcal{C}$ with Serre functor and non-zero projective objects are exactly the categories $\widetilde{\operatorname{rep}}(Q)$, where $Q$ is a connected quiver which is a star. 


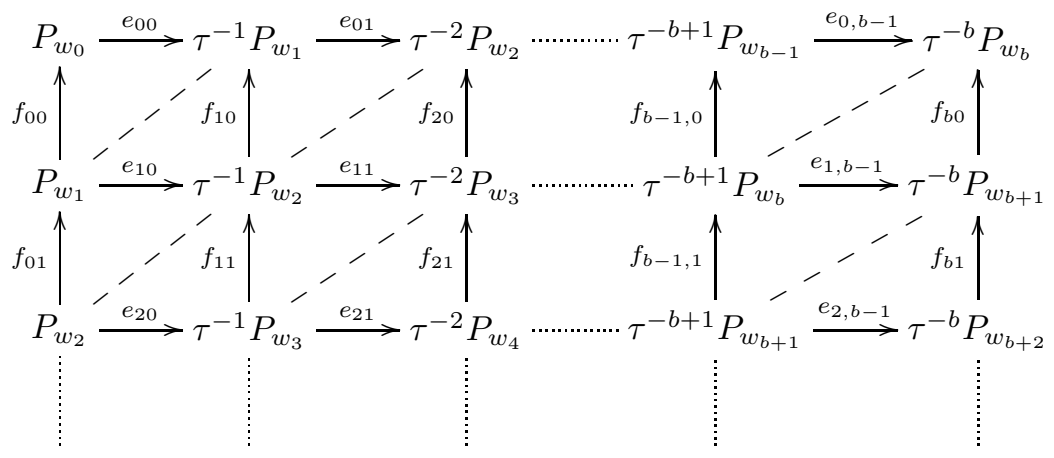

FiguRE II.4.1. Part of the preprojective component of $\widetilde{\operatorname{rep}}(Q)$

\section{SOURCES OF HEREDITARY ABELIAN CATEGORIES WITH NO PROJECTIVES OR INJECTIVES}

In this section we give various sources of examples of hereditary abelian categories. The main focus is on the Ext-finite categories which are noetherian and have a Serre functor, and we shall see in the next section that our discussion includes all possible examples with no non-zero projectives. It is also interesting to see how these examples fit into more general classes of hereditary abelian categories.

In $₫ I I .1$ we investigate hereditary abelian categories whose objects have finite length, and classify those having a Serre functor.

In $₫ I I .2$ we describe the hereditary abelian categories that arise as $\operatorname{qgr}(S)$ for $S$ a graded ring finite over its center (see III.2 for the definition of $\operatorname{qgr}(S)$ ).

In $₫$ III.3 we give examples of noetherian hereditary abelian categories obtained by tilting with respect to torsion pairs, performed inside the bounded derived category, as discussed in $\amalg .2$.

III.1. Hereditary abelian categories with Serre functor and all objects of finite length. When $\mathcal{C}$ is a hereditary abelian category, the subcategory f.l. $\mathcal{C}$ whose objects are those of finite length is again hereditary abelian, by Proposition A.2. In this section we classify the hereditary abelian categories $\mathcal{C}$ having a Serre functor, and where $\mathcal{C}=$ f.l.C.

Recall that $\tilde{A}_{n-1}$ denotes the graph which is a cycle with $n$ vertices. Then we have the following.

Theorem III.1.1. Let $\mathcal{C}$ be a connected Ext-finite noetherian hereditary abelian category in which every object has finite length and which has almost split sequences and no non-zero projectives or injectives. Then $\mathcal{C}$ is equivalent to the category of nilpotent finite dimensional representations of the quiver $\tilde{A}_{n-1}$ or of the quiver $A_{\infty}^{\infty}$, with all arrows oriented in the same direction. In the first case $n$ is the number of simple objects in $\mathcal{C}$ and in the second case there is an infinite number of simple objects.

Conversely, the category of nilpotent finite dimensional representations of the quiver $\tilde{A}_{n-1}$ or of the quiver $A_{\infty}^{\infty}$, with all arrows oriented in the same direction, is a noetherian hereditary abelian Ext-finite category with almost split sequences, and hence also with a Serre functor. 
Proof. By Theorem I.3.3 the category $\mathcal{C}$ has a Serre functor. As usual we denote the Serre functor by $F$, and we let $\tilde{\tau}=F[-1]$. This now induces an autoequivalence $\tau: \mathcal{C} \rightarrow \mathcal{C}$.

Let $S$ be an object in $\mathcal{C}$, and let $\mathcal{C}^{\prime}$ be the component of the AR-quiver of $\mathcal{C}$ containing $S$. Since $\tau: \mathcal{C} \rightarrow \mathcal{C}$ is an equivalence, it preserves length. Hence we have an almost split sequence $0 \rightarrow \tau S \rightarrow E \rightarrow S \rightarrow 0$, where $E$ is uniserial of length 2 . Consider the almost split sequence $0 \rightarrow \tau E \rightarrow \tau S \oplus F \rightarrow E \rightarrow 0$. Since $E$ and $\tau E$ are uniserial of length 2 and $\tau S$ is simple, it is easy to see that $F$ is uniserial of length 3 . Continuing the process, we see that $\mathcal{C}^{\prime}$ contains only uniserial objects. It is a tube if it contains only a finite number of non-isomorphic simple objects, and of the form $\mathbb{Z} A_{\infty}$ otherwise.

Assume there is some indecomposable object $X$ in $\mathcal{C}$ which is not in $\mathcal{C}^{\prime}$. If $\operatorname{Hom}\left(X, \mathcal{C}^{\prime}\right) \neq 0$, then $\operatorname{Hom}(X, S) \neq 0$ for some simple object $S$ in $\mathcal{C}^{\prime}$. Using the properties of almost split sequences and that all objects in $\mathcal{C}^{\prime}$ are uniserial, we can lift the map $f: X \rightarrow S$ to get an epimorphism from $X$ to an object of arbitrary length in $\mathcal{C}^{\prime}$. Hence $\operatorname{Hom}\left(X, \mathcal{C}^{\prime}\right)=0$, and similarly $\operatorname{Hom}\left(\mathcal{C}^{\prime}, X\right)=0$. It follows that all indecomposable objects in $\mathcal{C}$ are in $\mathcal{C}^{\prime}$.

It is easy to see from the above that the component of $\mathcal{C}$ uniquely determines $\mathcal{C}$ and that $\mathcal{C}$ is as in the statement of the theorem.

The category of nilpotent finite dimensional representations of $\tilde{A}_{n-1}$ or of $A_{\infty}^{\infty}$ over $k$ is a hereditary abelian $k$-category, and it is easy to see that it has almost split sequences, and hence a Serre functor by Theorem I.3.3.

Note that in 29] the connected finite quivers $Q$ where f.l. $(\operatorname{Rep} Q)$ has almost split sequences are described to be the above quivers $\tilde{A}_{n}$ and the quivers $Q$ with no oriented cycles.

There are various alternative but equivalent descriptions of the category $\mathcal{C}$. For further reference we now give a discussion of those.

For $n>0$ we define $C$ as the ring of $n \times n$-matrices of the form

$$
\left(\begin{array}{cccc}
R & R x & \cdots & R x \\
\vdots & \ddots & \ddots & \vdots \\
\vdots & & \ddots & R x \\
R & \cdots & \cdots & R
\end{array}\right)
$$

with $R=k[[x]]$. We put the standard $(x)$-adic topology on $C$. Since $C$ is the completed path algebra of $\tilde{A}_{n-1}$, it follows that $\mathcal{C}$ is equivalent to the category of finite dimensional representations over $C$.

Similarly for $A_{\infty}^{\infty}$ let $R=k[x]$ considered as a graded $\operatorname{ring}$ with $\operatorname{deg} x=1$. It is easily seen that $\mathcal{C}$ is now equivalent to $\operatorname{tors}(R)$, which consists of the finite dimensional $R$-modules.

In order to have compatibility with the first case let $C$ be the ring of lower triangular $\mathbb{Z} \times \mathbb{Z}$-matrices over $k$, equipped with the product of the discrete topologies on $k$. Thus $C$ is a pseudo-compact ring in the sense that its topology is complete and separated and in addition is generated by ideals of finite colength. For details on pseudo-compact rings see [11, 32, 33. Since $C$ is the completed path algebra of $A_{\infty}^{\infty}$, it follows that $\mathcal{C}$ is equivalent to the category of pseudo-compact finite dimensional representations over $C$. Note that one can prove (as in the proof of 
33, Thm. 1.1.3]) that all finite dimensional $C$-representations equipped with the discrete topology are pseudo-compact (this depends on $|k|=\infty$ ).

Let $\operatorname{dis}(C)$ be the category of finite dimensional $C$-modules (with the discrete topology). In the sequel we will denote the equivalence $\mathcal{C} \rightarrow \operatorname{dis}(C)$ by $(-)^{\text {r. To }}$ finish the description of $\mathcal{C}$ we describe the functor $\tau$.

Proposition III.1.2. There exists an invertible bi-pseudo-compact 32] C-bimodule $\omega_{C}$ such that for $T \in \mathcal{C}$ we have

$$
\widehat{\tau T}=\hat{T} \otimes_{C} \omega_{C} .
$$

Furthermore non-canonically we have $\omega_{C}=\operatorname{rad}(C)$.

Proof. Since Serre functors are unique we may assume $\mathcal{C}=\operatorname{dis}(C)$. It is easy to see that $\omega_{C}$ exists and is given by the formula

$$
\omega_{C}=\underset{I \subset C}{\operatorname{proj}} \lim \tau(C / I)
$$

where $I$ runs through the ideals of $C$ with the property that $C / I$ is finite dimensional. In fact such a formula would hold for any autoequivalence of $\mathcal{C}$.

To describe $\omega_{C}$ explicitly consider first the case $n<\infty$. In that case local duality implies that $\omega_{C} \cong \operatorname{Hom}_{R}\left(C, \omega_{R}\right)$ where $\omega_{R}=\Omega_{R}^{1}=R d x \cong R$. An explicit computation reveals that $\omega_{C} \cong \operatorname{rad}(C)$.

In the case of $A_{\infty}^{\infty}$ we could extend local duality theory to the pseudo-compact ring $C$ to arrive at the same formula. However it is easier to use that $\mathcal{C} \cong \operatorname{tors}(R)$ with $R=k[x]$. In this case graded local duality implies that $\tau$ coincides with tensoring with $\omega_{R}=\Omega_{R}^{1}=R d x \cong R(-1)$. Translating this to $C$ we see that indeed $\tau$ coincides with tensoring with $\operatorname{rad}(C)$.

We denote by $\mathrm{PC}(C)$ the category of pseudo-compact $C$-modules. $\mathrm{PC}(C)$ is an abelian category with enough projectives and exact inverse limits. Note the following fact which will be used in the sequel.

Lemma III.1.3. The homological dimension of $\mathrm{PC}(C)$ is one.

Proof. In [32, Cor. 4.7,4.11] it has been shown that it is sufficient to prove that the projective dimension of each pseudo-compact simple is equal to one. This is an easy direct verification.

III.2. Sheaves of hereditary orders and graded rings. Let $X$ be a nonsingular projective curve over $k$. Let $K$ be the function field of $X$, and let $A=$ $M_{n}(K)$ for some $n>0$. Let $\mathcal{O}$ be a sheaf of hereditary $\mathcal{O}_{X}$-orders in $A$. Thus locally $\mathcal{O}$ is a hereditary order over a Dedekind ring (in the sense of [21]). Let $\mathcal{C}=\operatorname{coh}(\mathcal{O})$ be the category of coherent $\mathcal{O}$-modules. Then $\mathcal{C}$ is obviously hereditary. Put $\omega=\mathcal{H o m}_{\mathcal{O}_{X}}\left(\mathcal{O}, \omega_{X}\right)$. Then exactly as in the commutative case one proves for $\mathcal{E}, \mathcal{F} \in \mathcal{C}$ :

$$
\operatorname{Hom}_{\mathcal{O}}(\mathcal{E}, \mathcal{F}) \cong \operatorname{Ext}_{\mathcal{O}}^{1}\left(\mathcal{F}, \mathcal{E} \otimes_{\mathcal{O}} \omega\right)^{*} .
$$

Hence $\mathcal{C}$ satisfies Serre duality.

From the structure of hereditary orders 21] it follows that every point $x \in X$ corresponds to a unique $-\otimes \omega$ orbit of simples (those simples with support in $x$ ). Hence in particular $\mathcal{C}$ has an infinite number of $\tau$-orbits of simples. 
At some point in the proof of Theorem IV.5.2 we need that if $S$ is a commutative graded ring such that $\operatorname{qgr}(S)$ is connected hereditary abelian, then $\operatorname{qgr}(S)$ is of the form $\operatorname{coh}(\mathcal{O})$ for $\mathcal{O}$ as above. Here $\operatorname{qgr}(S)$ denotes the quotient category $\operatorname{gr}(S) /$ finite length. We prove this in the larger generality that $S$ is finite over its center. Once we have the classification in Theorem IV.5.2 available we will be able to give a proof under even more general conditions on $S$. See Corollary V.2.2.

We start with the following preliminary result.

Proposition III.2.1. Let $S$ be a noetherian $\mathbb{Z}$-graded $k$-algebra finite dimensional over $k$ in every degree with left limited grading and which is finitely generated as a module over a central commutative ring $C$. Let $X=\operatorname{Proj} C$ (see [17] for the definition of Proj). Then $\operatorname{qgr}(S)$ is equivalent to the category of coherent modules over a sheaf $\mathcal{O}$ of $\mathcal{O}_{X}$-algebras which is coherent as $\mathcal{O}_{X}$-module.

Proof. Without changing the category $\operatorname{qgr}(S)$ we may (and we will) replace $S$ by $S=k+S_{1}+S_{2}+\cdots$. By the Artin-Tate lemma $C$ is finitely generated. Let $x_{1}, \ldots, x_{n}$ be homogeneous generators for $C$, respectively of degree $a_{1}, \ldots, a_{n}>0$. Let $m_{1}, \ldots, m_{t}$ be homogeneous generators of $S$ as a module over $C$, and let $e$ be the maximum of the degrees of the $m$ 's. Let $p$ be the product of the $a_{i}$ 's. We claim that there exists $b_{0} \in \mathbb{N}$ such that for $b \geq b_{0}$ we have $S_{p} S_{b}=S_{p+b}$.

We prove instead that for $a$ large we have $S_{a}=S_{p} S_{a-p}$. This is clearly equivalent. A general element of $S_{a}$ is a $k$-linear combination of elements of the form $s=x_{1}^{p_{1}} \cdots x_{n}^{p_{n}} m_{j}$. It is sufficient to have that for some $i: p_{i} \geq a_{1} \cdots \hat{a}_{i} \cdots a_{n}$. Assume this is false. Then it follows that the degree of $s$ (which is $a$ ) is less than $n p+e$. This proves what we want.

Define

$$
T=\left(\begin{array}{cccc}
S & S(1) & \cdots & S(p-1) \\
S(-1) & S & \cdots & S(p-2) \\
\vdots & \vdots & \ddots & \vdots \\
S(-p+1) & S(-p+2) & \cdots & S
\end{array}\right)
$$

One verifies directly that for $b \geq p+b_{0}-1$ one has $T_{1} T_{b}=T_{b+1}=T_{b} T_{1}$. Since $S$ and $T$ are Morita equivalent, we clearly have $\operatorname{qgr}(S)=\operatorname{qgr}(T)$.

By definition $X$ is covered by the affine open sets $U_{i}=\operatorname{Spec}\left(C_{x_{i}}\right)_{0}$. Let $\mathcal{T}$ be the sheaf of graded rings on $X$ associated to $T$. Thus on $U_{i}$ the sections of $\mathcal{T}$ are given by $T_{x_{i}}$.

By localization theory $\operatorname{qgr}(T)$ is equivalent to the category of coherent graded $\mathcal{T}$-modules. We claim that $\mathcal{T}$ is strongly graded $\left(\mathcal{T}_{a} \mathcal{T}_{b}=\mathcal{T}_{a+b}\right)$ and that $\mathcal{O}=\mathcal{T}_{0}$ is coherent over $\mathcal{O}_{X}$. It is clearly sufficient to check this locally. That $T_{x_{i}}$ is strongly graded follows from Lemma III.2.2 below.

The sheaf $\mathcal{O}$ is nothing but $\tilde{T}$ in the notation of [17]. By the results in loc. cit. $\tilde{T}$ is coherent (it is easy to see this directly).

Since $\mathcal{T}$ is strongly graded, we have that the category of coherent graded $\mathcal{T}$ modules is equivalent to the category of coherent $\mathcal{O}$-modules. This finishes the proof.

Lemma III.2.2. Assume that $B$ is a $\mathbb{Z}$-graded ring such that $B_{1} B_{b}=B_{b+1}=$ $B_{b} B_{1}$ for large $b$, and assume furthermore that there exists a non-zero $r>0$ such that $B_{-b r} B_{b r}=B_{0}$ for all $b$. Then $B$ is strongly graded. 
Proof. For $b$ large we have

$$
B_{1} B_{-1} \supset B_{1} B_{b r-1} B_{-b r}=B_{b r} B_{-b r}=B_{0} .
$$

A similar argument shows that $\mathcal{B}_{-1} B_{1}=B_{0}$.

We can now prove the desired result.

Proposition III.2.3. Let $S$ be as in Proposition III.2.1 and assume in addition that $\operatorname{qgr}(S)$ is hereditary. Then $\operatorname{qgr}(S)$ is a finite direct sum of hereditary abelian categories which are either of the form $\bmod (\Lambda)$ for a finite dimensional algebra $\Lambda$, or else of the form $\operatorname{coh}(\mathcal{O})$ where $\mathcal{O}$ is a sheaf of classical hereditary orders over a non-singular irreducible projective curve $Y$.

Proof. This follows easily from the structure theory of hereditary noetherian rings.

By Proposition $\amalg I .2 .1$ it follows that $\operatorname{qgr}(S)$ is equivalent to the category of coherent $\mathcal{O}$-modules where $\mathcal{O}$ is a coherent sheaf of $\mathcal{O}_{X}$-algebras and $X=\operatorname{Proj} C$.

Let $\mathcal{Z}$ be the center of $\mathcal{O}$, and let $\pi: Y \rightarrow X$ be the $X$-scheme such that $\pi_{*} \mathcal{O}_{Y}=\mathcal{Z}$. Now for the purposes of the proof we may replace $Y$ by its connected components. So we assume that $Y$ is connected. Let $U \subset Y$ be affine open. Then by Proposition A.4 and [11, p. 431] it follows that $\mathcal{O}(U)$ is hereditary. So according to [20, p. 151] we have that $\mathcal{O}(U)$ will be a direct sum of a finite dimensional hereditary algebra $\Lambda$ and a hereditary $\mathcal{O}(U)$-algebra $H$ which is a direct sum of infinite dimensional prime rings. We identify $\Lambda$ with a sheaf of finite support on $Y$. If $\Lambda \neq 0$, then $\Lambda$ corresponds to a central idempotent in $\mathcal{O}(U)$ which is zero outside the support of $\Lambda$. Hence we can extend it to a central idempotent in $\mathcal{O}$. Since we had assumed that the center of $\mathcal{O}$ is connected, this yields $\mathcal{O}=\Lambda$.

Hence we only have to consider the case where $\mathcal{O}(U)$ is a direct sum of prime hereditary rings for all affine $U \subset Y$. In our case the $\mathcal{O}(U)$ are then direct sums of orders in central simple algebras.

Now it follows from [26] that the center of a hereditary order is a Dedekind ring. In particular $Y$ is a non-singular curve. Since $Y$ is also connected, it follows that $Y$ is irreducible. Thus if $U \subset Y$ is affine, then $\mathcal{O}_{Y}(U)$ is a domain, whence $\mathcal{O}(U)$ is actually prime. So $\mathcal{O}(U)$ is a classical hereditary order. This finishes the argument.

One of the conditions of the previous proposition was that $\operatorname{qgr}(S)$ should be hereditary. The following lemma, which is almost a tautology (using Proposition A.3), tells us when this condition holds.

Lemma III.2.4. With the above hypotheses on $S$ we have that $\operatorname{qgr}(S)$ is hereditary if and only if $S_{P}$ is hereditary for every non-maximal graded prime ideal $P$ in the center of $S$.

III.3. Hereditary abelian categories associated to infinite Dynkin and tame quivers. In this section we construct some particular hereditary abelian noetherian categories which are obtained from categories of type $\operatorname{rep}(Q)$ via tilting with respect to some torsion pair.

We start by computing the AR-quivers for the finite dimensional representations of the quivers $A_{\infty}^{\infty}, D_{\infty}$ and $A_{\infty}$ (with respect to a convenient orientation). The first two are needed for the construction of noetherian hereditary abelian categories with no non-zero projectives and the third will be useful for giving an example that in the non-noetherian case a connected hereditary abelian Ext-finite category $\mathcal{C}$ 


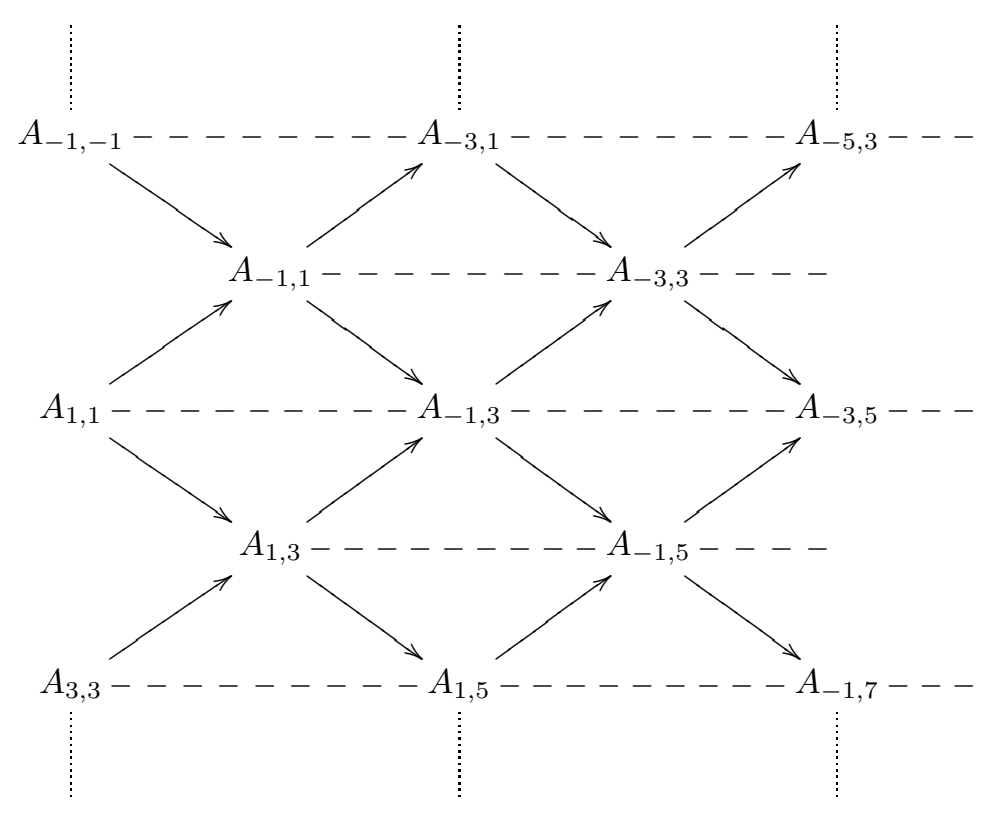

FIgURE III.3.1. The preprojective component of $A_{\infty}^{\infty}$

with Serre duality and non-zero projectives is not necessarily determined by its associated quiver.

III.3.1. The case of $A_{\infty}^{\infty}$. We consider the category of finite dimensional representations of the quiver

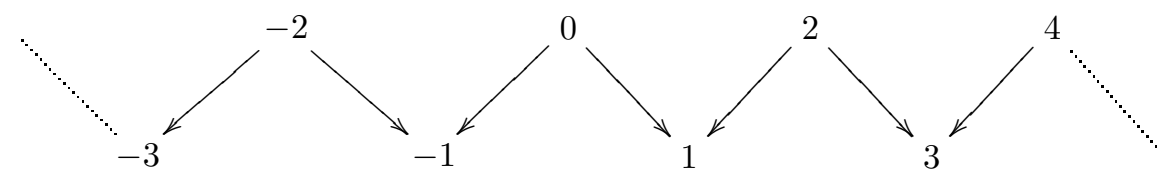

of type $A_{\infty}^{\infty}$. Since each representation can be viewed as a representation of some quiver of type $A_{n}$, we know the structure of the indecomposable representations. We recall what they look like and at the same time we introduce a convenient notation:

$A_{n, m}(n \leq m): V_{i}=k$ for $n \leq i \leq m$ and $V_{i}=0$ otherwise. Then the AR-quiver contains components given by Figures 11.3 .1 ஹ.3.2 ஹ.3.3 ஹ.3.4 respectively.

To see that the corresponding sequences are almost split we use the known structure of almost split sequences for quivers of type $A_{n}$.

We see that the preprojective component contains the $A_{n, m}$ with $m, n$ odd, the preinjective component contains those with $m, n$ even, the $\mathbb{Z} A_{\infty}$ component in Figure $\amalg I .3 .3$ contains the $A_{n, m}$ with $n$ even and $m$ odd and finally the $\mathbb{Z} A_{\infty}$ component in Figure III.3.4 contains the $A_{n, m}$ with $n$ odd and $m$ even.

Hence all indecomposable representations occur in one of the four above components, and so there are no other components. 


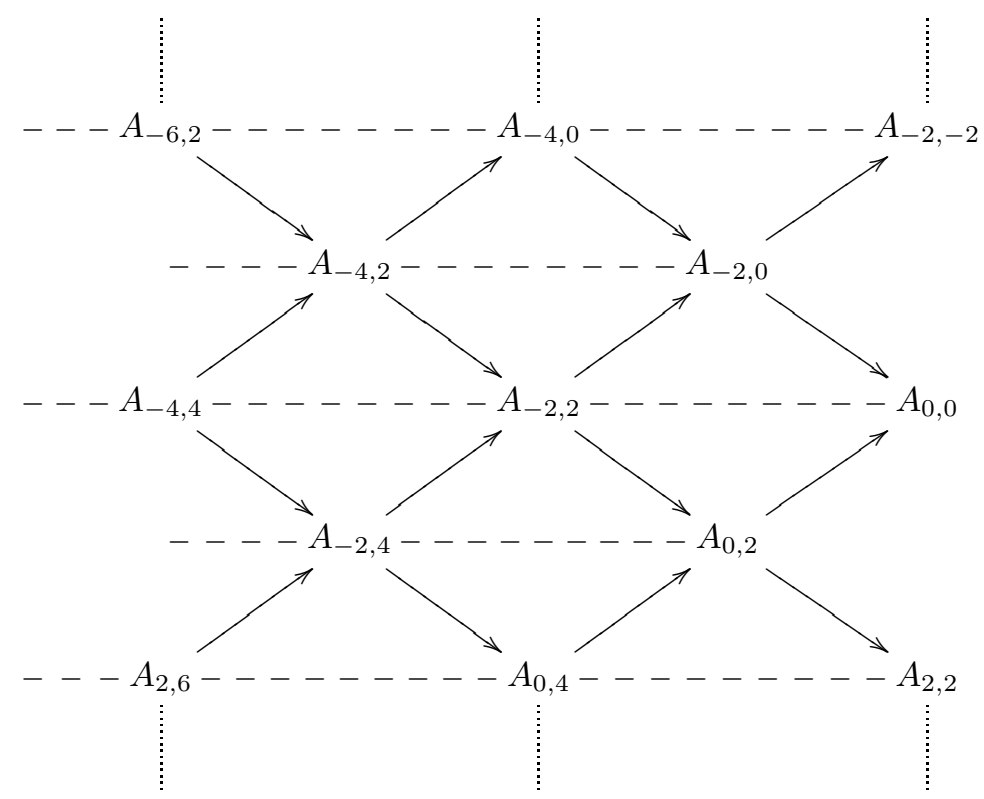

FiguRE III.3.2. The preinjective component of $A_{\infty}^{\infty}$

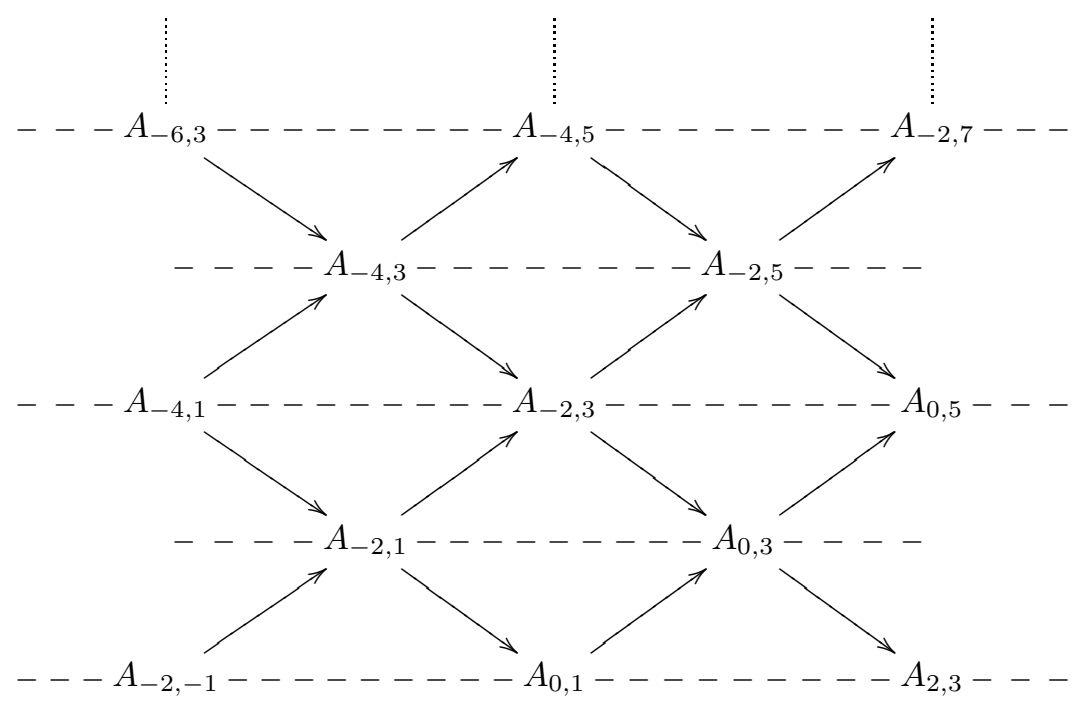

Figure III.3.3. The first $\mathbb{Z} A_{\infty}$ component of $A_{\infty}^{\infty}$ 


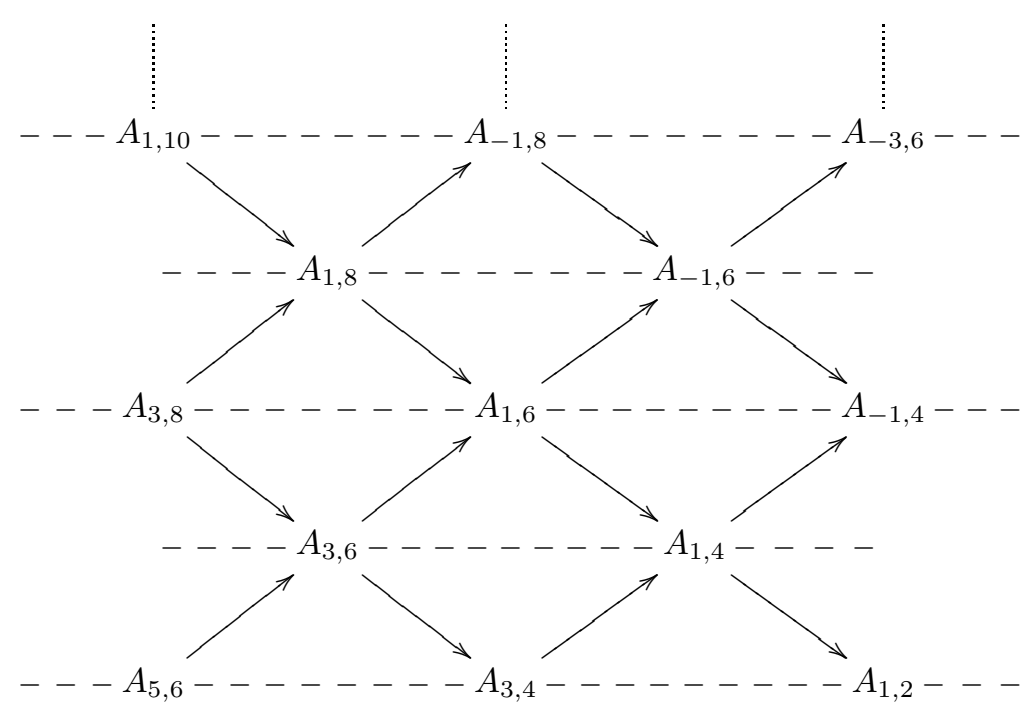

FigURE III.3.4. The second $\mathbb{Z} A_{\infty}$ component of $A_{\infty}^{\infty}$

III.3.2. The case of $D_{\infty}$. We consider the category of finite dimensional representations for the quiver

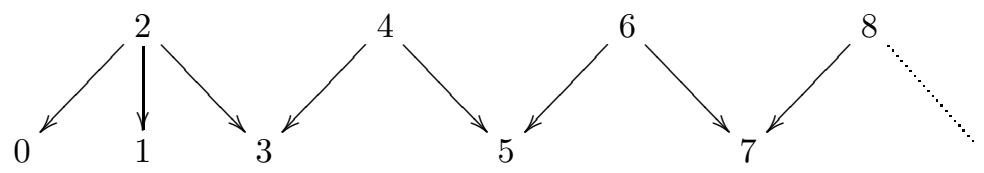

of type $D_{\infty}$. Since each finite dimensional representation can be viewed as a representation of some quiver of type $D_{n}$, we know the structure of the indecomposable representations. We recall what they look like, and at the same time we introduce a notation convenient for describing the distribution of modules in the AR-quiver (we always mean that $V_{j}=0$ at the vertices which are not mentioned).

$$
\begin{aligned}
A_{n, m}: & V_{i}=k \text { for } 2 \leq n \leq i \leq m . \\
A_{m}^{(1)}: & V_{i}=k \text { for } i \leq m, i \neq 1, V_{1}=0 . \\
A_{m}^{(0)}: & V_{i}=k \text { for } i \leq m, i \neq 0, V_{0}=0 . \\
B_{n, m}: & V_{i}=k^{2} \text { for } 2 \leq i \leq n, V_{i}=k \text { for } i=0,1, \text { and } n+1 \leq i \leq m \text { (for } \\
& 1 \leq n \leq m, 2 \leq m) .
\end{aligned}
$$

We now compute three components of the AR-quiver (see Figures III.3.5, III.3.6 III.3.7). For those people that are having trouble spotting the rule for Figure III.3.7 it may help to introduce the convention $B_{n, m}=B_{m, n}$.

It is not hard to see that the sequences corresponding to Figures II.3.5 W.3.6 III.3.7 are almost split by considering a large enough subcategory of representations of a quiver of type $D_{n}$.

We see that the preprojective component contains the $A_{n, m}, A_{m}^{(0)}$ and $A_{m}^{(1)}$ with $m$ and $n$ odd or 0 and the $B_{n, m}$ with $n, m$ odd. The preinjective component contains the $A_{n, m}, A_{m}^{(0)}$ and $A_{m}^{(1)}$ with $m$ even and $n$ even $\neq 0$ and the $B_{n, m}$ with $n, m$ even. 


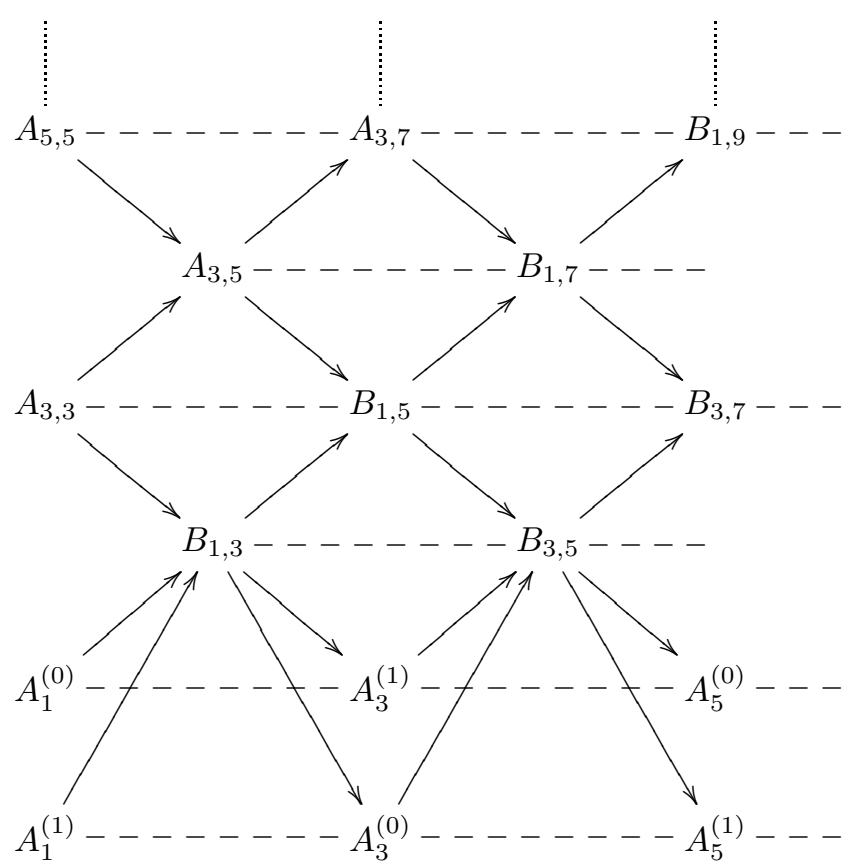

FiguRE III.3.5. The preprojective component of $D_{\infty}$

The third component contains the $A_{n, m}$ and $B_{n, m}$ with $n+m$ odd and the $A_{0, m}$ with $m$ even.

Hence all indecomposable representations lie in one of the above three components, and hence there are no other components.

III.3.3. The case of $A_{\infty}$. We consider the category of finite dimensional representations of the quiver
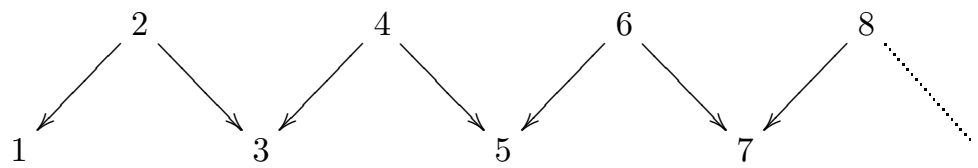

of type $A_{\infty}$. We use the same notation as in the case $A_{\infty}^{\infty}$ for the indecomposable representations. Then the AR-quiver contains components given by Figures $\amalg I I .3 .8$ III.3.9 respectively. The $A_{n, m}$ with $m$ odd are in the preprojective component, and those with $m$ even are in the preinjective component. Hence there are no other components.

III.3.4. The $\mathbb{Z} A_{\infty}^{\infty}$ category. Let $\mathcal{C}$ be $\operatorname{rep}(Q)$ where $Q$ is the quiver $A_{\infty}^{\infty}$ with zigzag orientation (as in $₫$ III.3.1), and let $\mathcal{C}_{1}$ be obtained from $\mathcal{C}$ by tilting with respect to the split torsion pair $(\mathcal{T}, \mathcal{F}$ ), where $\mathcal{T}$ consists of the preinjectives (see Figure $\llbracket .3 .2$ ). Then $\mathcal{C}_{1}$ is an Ext-finite hereditary abelian category with no non-zero projectives or injectives, derived equivalent to $\mathcal{C}$. So $\mathcal{C}_{1}$ satisfies Serre duality. The AR-quiver of $\mathcal{C}_{1}$ consists of one $\mathbb{Z} A_{\infty}^{\infty}$ component $\Sigma$ and two $\mathbb{Z} A_{\infty}$ components. 


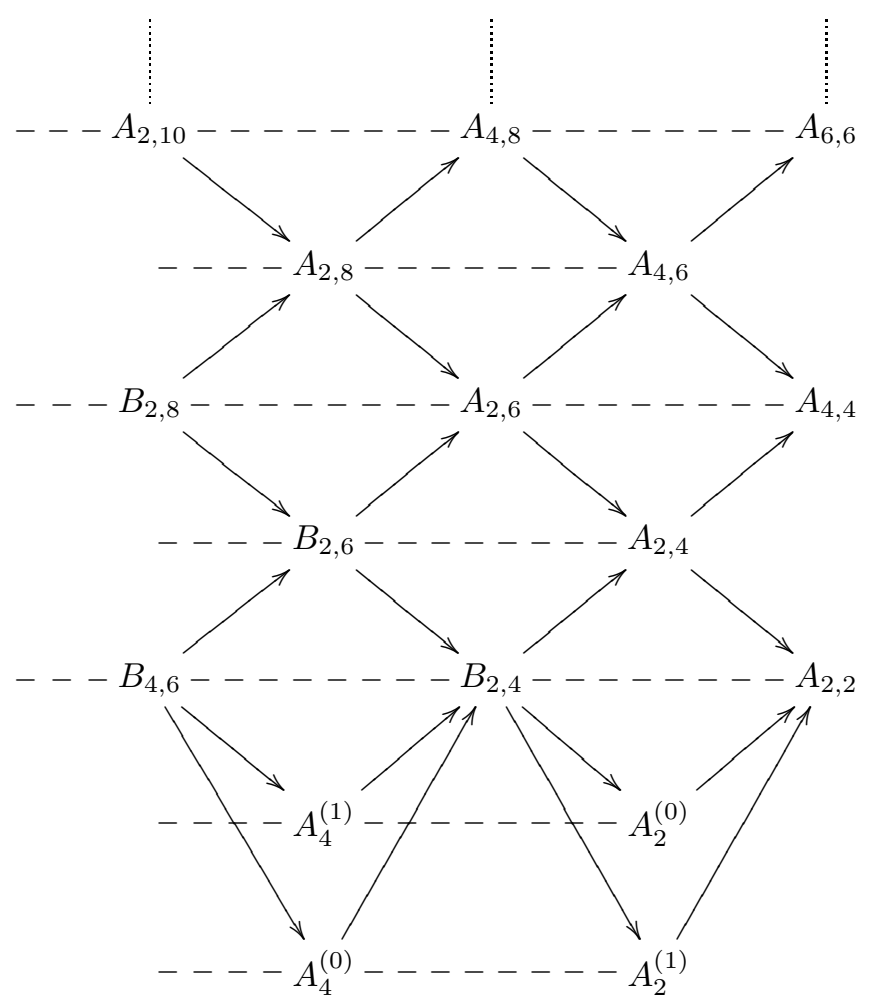

FIgURE III.3.6. The preinjective component of $D_{\infty}$

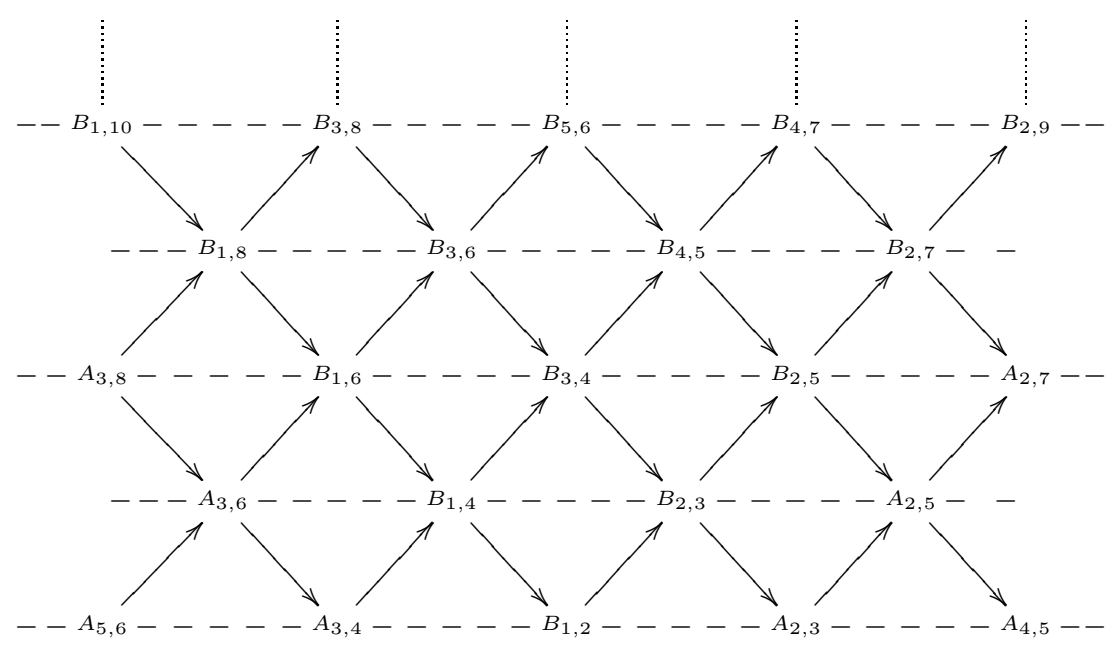

Figure III.3.7. The $\mathbb{Z} A_{\infty}$ component of $D_{\infty}$ 


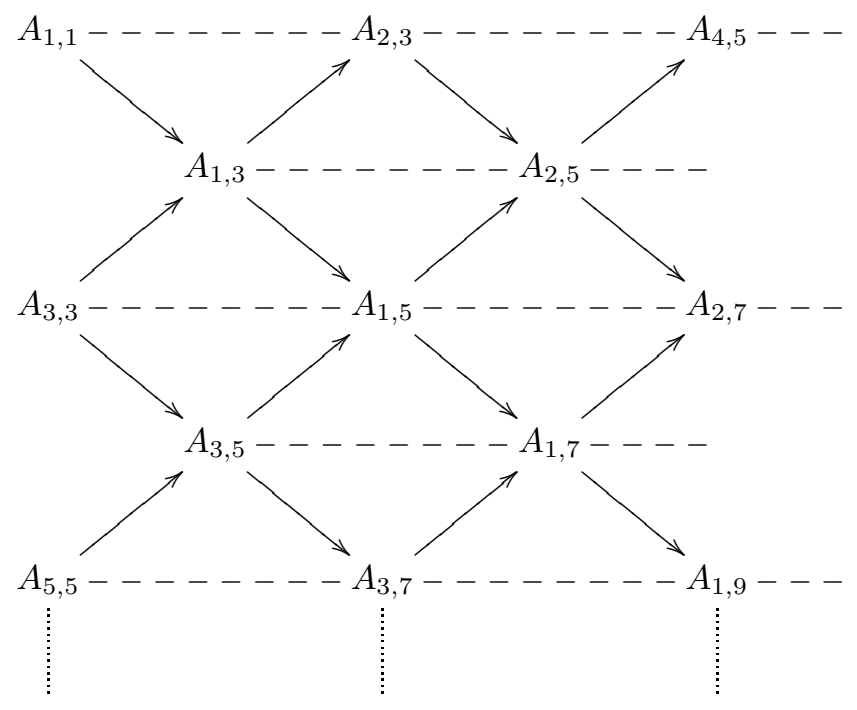

Figure III.3.8. The preprojective component of $A_{\infty}$

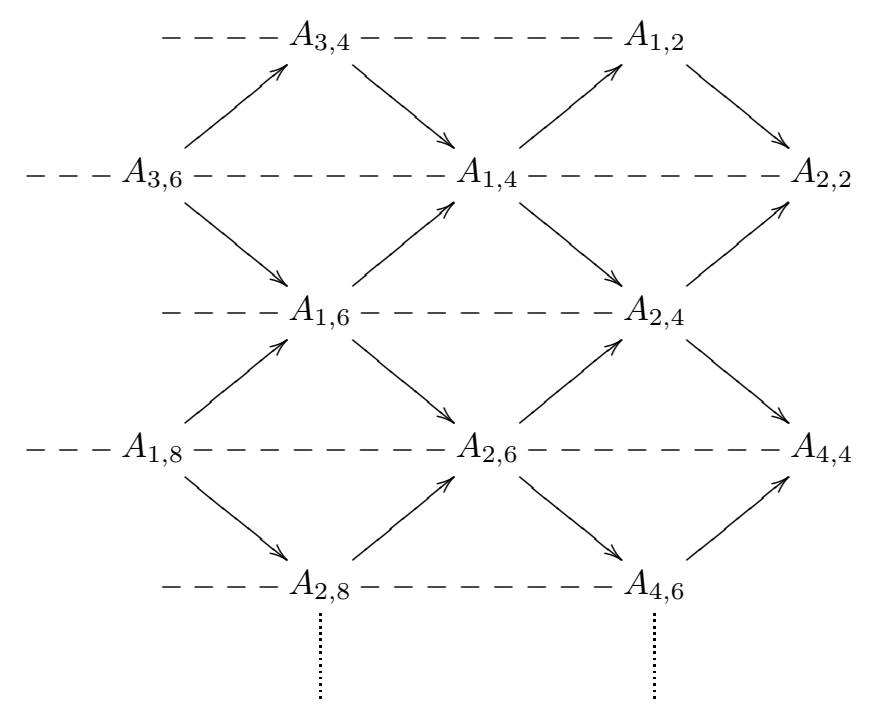

Figure III.3.9. The preinjective component of $A_{\infty}$

Below we show that $\mathcal{C}_{1}$ is noetherian and we show in addition that $\mathcal{C}_{1}$ has two $\tau$-orbits of simples. But first we give some preliminary results. 
In an $A R$-quiver the subquiver of the form

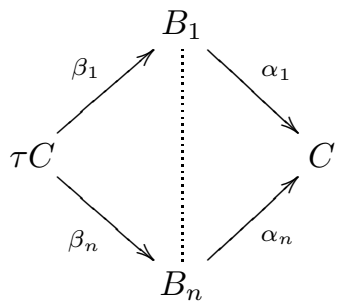

given by an almost split sequence $0 \rightarrow \tau C \rightarrow B_{1} \oplus \cdots \oplus B_{n} \rightarrow C \rightarrow 0$, with the $B_{i}$ indecomposable (and which we here assume to be non-isomorphic), is called a mesh. The mesh category of a component $\mathcal{C}^{\prime}$ is the path category of $\mathcal{C}^{\prime}$ modulo the relations $\sum_{i=1}^{n} \alpha_{i} \beta_{i}$ given by the meshes. The component $\mathcal{C}^{\prime}$ of an AR-quiver is said to be standard if the full subcategory of indecomposable objects corresponding to the vertices of $\mathcal{C}^{\prime}$ is equivalent to the mesh category of $\mathcal{C}^{\prime}$.

Lemma III.3.4.1. Let $\mathcal{C}$ and $\mathcal{C}_{1}$ be as above.

1. Let $X$ and $Y$ be indecomposable objects belonging to a component of the ARquiver of $\mathcal{C}$ or $\mathcal{C}_{1}$. If $f: X \rightarrow Y$ is a non-zero non-isomorphism, then $f$ is a composition of irreducible maps. In particular there is a path between the corresponding vertices.

2. The components of the $A R$-quiver of $\mathcal{C}$ and of $\mathcal{C}_{1}$ are standard.

Proof. Part 1. for $\mathcal{C}$ follows directly from the explicit description of the indecomposable objects in $\mathcal{C}$. The claim for $\mathcal{C}_{1}$ can be reduced to the result for $\mathcal{C}$, by possibly applying high enough powers of $\tau^{-1}$.

To show that any component $\mathcal{C}^{\prime}$ of $\mathcal{C}$ or $\mathcal{C}_{1}$ is standard, one defines as usual a functor from the path category of $\mathcal{C}^{\prime}$ to the subcategory of indecomposable objects of $\mathcal{C}$ given by $\mathcal{C}^{\prime}$, in such a way that there is induced a functor from the mesh category of $\mathcal{C}^{\prime}$. Using 1 ., this will be an equivalence.

The next result, which gives information on maps between components, is easily verified.

Lemma III.3.4.2. Let $\mathcal{C}$ and $\mathcal{C}_{1}$ be as above.

1. For $\mathcal{C}$ there are no non-zero maps from the preinjective component to any other component and to the preprojective component from any other component, and also no non-zero maps between the two $\mathbb{Z} A_{\infty}$ components.

2. For $\mathcal{C}_{1}$ there are no non-zero maps from the $\mathbb{Z} A_{\infty}$ components to the $\mathbb{Z} A_{\infty}^{\infty}$ component, and no non-zero maps between the two $\mathbb{Z} A_{\infty}$ components.

We state explicitly the following special cases which will be used later.

Lemma III.3.4.3. Let $\mathcal{C}_{1}$ be as above.

1. If $U$ and $W$ are indecomposable with $\operatorname{Hom}(W, U) \neq 0$ and $U$ is in the $\mathbb{Z} A_{\infty}^{\infty}$ component of $\mathcal{C}_{1}$, then there is a path from $W$ to $U$ in the AR-quiver.

2. If $X$ is indecomposable in $\mathcal{C}_{1}$, then $\operatorname{End}_{\mathcal{C}_{1}}(X) \simeq k$.

Proposition III.3.4.4. $\mathcal{C}_{1}$ has exactly two $\tau$-orbits of simples.

Proof. We claim that the only simple objects in $\mathcal{C}_{1}$ are the objects of length two in the $\mathbb{Z} A_{\infty}$ components of $\mathcal{C}$. This clearly implies what we have to show. 
First we note that the other indecomposables cannot be simple. Indeed let $X$ be one of those other indecomposables. Assume first that $X \in \mathcal{F}$. Thus $X$ can be considered as an object of $\mathcal{C}$. Assume now in addition that $X$ is not a length one projective. Then it is easy to see directly that we can make a non-trivial short exact sequence

$$
0 \rightarrow U \rightarrow X \rightarrow W \rightarrow 0
$$

with $U, W \in \mathcal{F}$. Hence this sequence remains exact in $\mathcal{C}_{1}$, and thus $X$ is not simple.

If $X$ happens to lie in $\mathcal{T}[-1]$, or is given by a length one projective in $\mathcal{C}$, then we first apply a power of $\tau^{-1}$, and then we apply the above reasoning.

Now we prove the converse. So let $X$ be an indecomposable object of length two in one of the $\mathbb{Z} A_{\infty}$ components of $\mathcal{C}$. We have to prove that $X$ becomes simple in $\mathcal{C}_{1}$.

Assume now that $X$ is not simple in $\mathcal{C}_{1}$. Thus there is an exact sequence in $\mathcal{C}_{1}$ as in (III.3.1), but this time the indecomposable summands of $W$ must be in the same component as $X$ (by Lemma III.3.4.2). By applying a high power of $\tau^{-1}$ we may assume that the summands of $U$ are in $\mathcal{F}$, and hence (III.3.1) represents an exact sequence in $\mathcal{C}$. It is easy to see that this is impossible.

Lemma III.3.4.5. As in Figure II.3.2 let $\Sigma$ be the $\mathbb{Z} A_{\infty}^{\infty}$ component of $\mathcal{C}_{1}$. Then the objects in $\Sigma$ generate $\mathcal{C}_{1}$.

Proof. This follows directly from the fact that the projectives generate $\mathcal{C}$.

Lemma III.3.4.6. One has for $X, Y \in \Sigma$

$$
\operatorname{dim} \operatorname{Hom}_{\mathcal{C}_{1}}(X, Y)= \begin{cases}1 & \text { if there is a path from } X \text { to } Y \\ 0 & \text { otherwise. }\end{cases}
$$

Proof. This can be shown in many ways. The most direct method is to compute in $\mathcal{C}$, but it is most elegant to consider the function $\phi_{X}=\operatorname{dim} \operatorname{Hom}(X,-)$. This function is 1 on $X$ and zero on the objects which are not on a path starting in $X$ (by Lemma III.3.4.3). Furthermore $\phi_{X}$ is additive on almost split sequences not ending in $X$. This is enough to determine $\phi_{X}$ completely.

We conclude:

Lemma III.3.4.7. If $X \in \Sigma$, then $X$ does not contain a non-trivial direct sum.

Proof. Assume $Y \oplus Z \subset X$ with $Y, Z$ indecomposable. Then we can find an indecomposable $C$ which has a path to both $Y$ and $Z$. Thus we obtain.

$$
1=\operatorname{dim} \operatorname{Hom}(C, X) \geq \operatorname{dim} \operatorname{Hom}(C, Y)+\operatorname{dim} \operatorname{Hom}(C, Z)=2
$$

which is a contradiction.

Proposition III.3.4.8. The category $\mathcal{C}_{1}$ is noetherian.

Proof. By LemmaIII.3.4.5it suffices to prove that the objects in $\Sigma$ are noetherian. So let $C$ be an object in $\Sigma$, and assume that $C$ is not noetherian. Then there is some infinite chain

$$
C_{0} \subsetneq C_{1} \subsetneq C_{2} \subsetneq \cdots \subset C .
$$

By Lemma II.3.4.7 each of the $C_{i}$ is indecomposable, and by Lemma II.3.4.2 each of the $C_{i}$ is in $\Sigma$. Thus by Lemma III.3.4.3 each of the $C_{i}$ is on a path from 
$C_{0}$ to $C$. Since there are only a finite number of objects on such paths, this is a contradiction.

III.3.5. The $\mathbb{Z} D_{\infty}$ category. This section closely parallels the previous one, but some of the arguments are slightly more complicated. Let $\mathcal{C}$ be $\operatorname{rep}(Q)$, where $Q$ is the quiver $D_{\infty}$ with zigzag orientation (as in 1 II.3.2. , and let $\mathcal{C}_{1}$ be obtained from $\mathcal{C}$ by tilting with respect to the split torsion pair $(\mathcal{T}, \mathcal{F})$, where $\mathcal{T}$ consists of the preinjectives (see Figure I.3.2). Then $\mathcal{C}_{1}$ is an Ext-finite hereditary abelian category with no non-zero projectives or injectives, derived equivalent to $\mathcal{C}$. So $\mathcal{C}_{1}$ satisfies Serre duality. The AR-quiver of $\mathcal{C}_{1}$ consists of one $\mathbb{Z} D_{\infty}$ component $\Sigma$ and one $\mathbb{Z} A_{\infty}$ component.

We want to show that $\mathcal{C}_{1}$ is noetherian, and that $\mathcal{C}_{1}$ has exactly one $\tau$-orbit of simples. Some of the preliminary results we need for this are similar to the corresponding results in III.3.4, and hence most of them are omitted.

We start with stating the following results, where the first one is of interest in itself and the second one is different from the corresponding result for the $\mathbb{Z} A_{\infty}^{\infty}$ category.

Lemma III.3.5.1. The components of $\mathcal{C}$ and $\mathcal{C}_{1}$ are standard.

Proposition III.3.5.2. $\mathcal{C}_{1}$ has exactly one $\tau$-orbit of simple objects.

Now we need an analog of Lemma II.3.4.6 but as is to be expected the result is slightly more complicated in the present case. Therefore the result is most easily represented in some diagrams. Figures 111.3 .10 and 11 .3.11 give the values of $\operatorname{dim} \operatorname{Hom}(X, Y)$ for $X, Y \in \Sigma$ where $X$ is varying and $Y$ is fixed. Figure III.3.11 corresponds to the case where $Y$ is on one of the ending vertices of $D_{\infty}$, and Figure III.3.10 corresponds to the general case. These diagrams are obtained in the same way as Lemma III.3.4.6 We have encircled the vertex corresponding to $Y$ in each of the cases.

From these diagrams we compute the following lemma.

Lemma III.3.5.3. If $X, Y \in \Sigma$, then $\operatorname{dim} \operatorname{Hom}(X, Y) \leq 2$.

This lemma allows us to prove the following.

Lemma III.3.5.4. If $X \in \Sigma$, then $X$ does not contain a non-trivial direct sum of three objects.

Proof. Assume $W \oplus Y \oplus Z \subset X$ with $W, Y, Z$ indecomposable. Then according to the diagrams $\amalg .3 .10$ and $\amalg .3 .11$ we can find an indecomposable $C$ such that $\operatorname{Hom}(C,-)$ is non-zero when evaluated on $W, Y$ and $Z$. Thus we obtain

$$
2 \geq \operatorname{dim} \operatorname{Hom}(C, X) \geq \operatorname{dim} \operatorname{Hom}(C, W)+\operatorname{dim} \operatorname{Hom}(C, Y)+\operatorname{dim} \operatorname{Hom}(C, Z) \geq 3
$$

which is a contradiction.

Proposition III.3.5.5. The category $\mathcal{C}_{1}$ is noetherian.

Proof. As in III.3.4 it suffices to prove that the objects in $\Sigma$ are noetherian. So let $C$ be an object in $\Sigma$, and assume that $C$ is not noetherian. Then there is some infinite chain

$$
C_{0} \subsetneq C_{1} \subsetneq C_{2} \subsetneq \cdots \subset C \text {. }
$$

After possibly replacing $\left(C_{i}\right)_{i}$ by a subsequence we may by Lemma ஹI.3.5.4 assume that either each of the $\left(C_{i}\right)_{i}$ is indecomposable, or else that they all have exactly two 


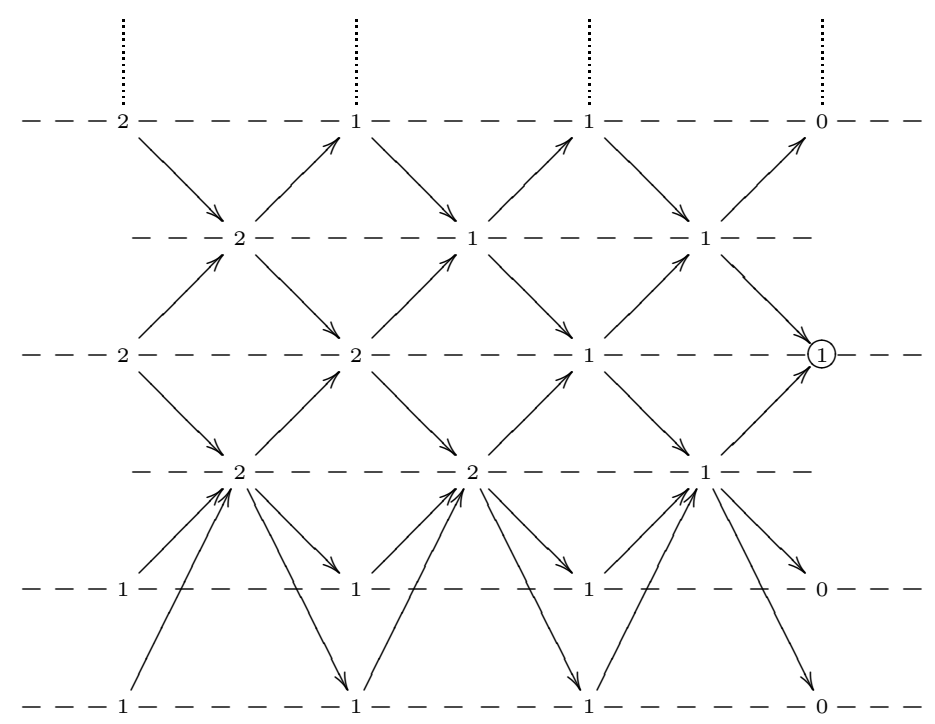

Figure III.3.10. $\operatorname{dim} \operatorname{Hom}(X, Y)$ for $X, Y \in \Sigma$ with $X$ variable

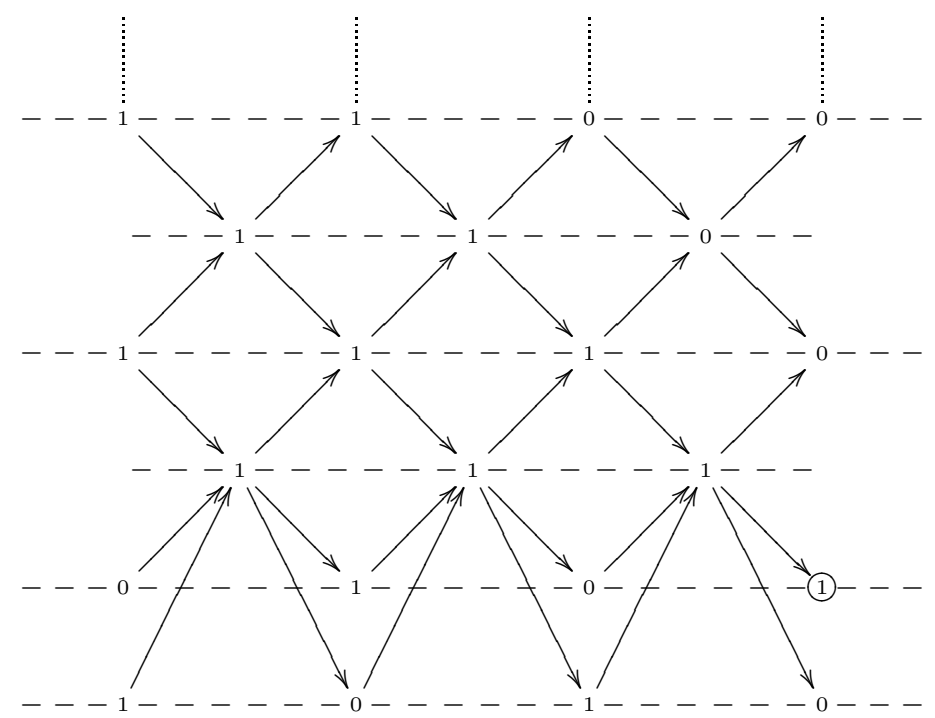

Figure III.3.11. $\operatorname{dim} \operatorname{Hom}(X, Y)$ for $X, Y \in \Sigma$ with $X$ variable

indecomposable summands. The first case is dealt with exactly as in Proposition III.3.4.8, so we will concentrate on the second case.

Thus we now assume that $C_{i}=D_{i} \oplus E_{i}$ with $D_{i}$ and $E_{i}$ indecomposable. Using Lemma III.3.5.4 it is easily seen that possibly after interchanging $D_{i+1}$ and $E_{i+1}$ we may assume that there exist non-zero maps $D_{i} \rightarrow D_{i+1}$ and $E_{i} \rightarrow E_{i+1}$. Thus as in the proof of Proposition III.3.4.8 we must have $D_{i} \cong D_{i+1}$ and $E_{i} \cong E_{i+1}$ 
for large $i$, and hence also $C_{i} \cong C_{i+1}$. From the fact that $\mathcal{C}_{1}$ is Hom-finite, it then easily follows that the inclusion $C_{i} \hookrightarrow C_{i+1}$ must actually be an isomorphism. This finishes the proof.

III.3.6. An example. We now give an example that shows that the important Theorem $\amalg .4 .2$ fails for non-noetherian hereditary abelian Ext-finite categories with Serre duality, even when they have noetherian injectives.

Let $\mathcal{C}_{1}$ be the $\mathbb{Z} A_{\infty}^{\infty}$ category as defined in $₫$ III.3.4. For one of the $Z A_{\infty}$ components consider a section $Q_{1} \subset \mathbb{Z} A_{\infty}$ with zig-zag orientation. Let $\mathcal{T}_{1}$ consist of direct sums of indecomposable objects which are in the chosen component to the right of $Q_{1}$, and let $\mathcal{F}_{1}$ consist of direct sums of the other indecomposables. Then it follows from $\amalg I$ II.3.4 together with Serre duality that $\left(\mathcal{T}_{1}, \mathcal{F}_{1}\right)$ is a split torsion pair in $\mathcal{C}_{1}$.

Let $\mathcal{D}$ be the tilted category. Then $\mathcal{D}$ is a hereditary abelian category whose projectives are given by $Q_{1}$ and which is derived equivalent to $\mathcal{C}_{1}$. It follows from Lemma $\amalg .3 .2$ that $\mathcal{D}$ has noetherian injectives. Hence if Theorem $\amalg .4 .2$ were to hold for $\mathcal{D}$, then by Corollary $\amalg .2 .8$ it would follow that $\mathcal{D}=\widetilde{\operatorname{rep}}\left(Q_{1}\right)=\operatorname{rep}\left(Q_{1}\right)$ (using the fact that $\mathcal{D}$, being derived equivalent to $\operatorname{rep}(Q)$, is indecomposable by construction). However this contradicts the determination of the AR-quiver of

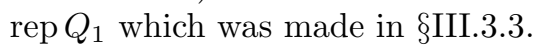

III.3.7. Other interpretations of the $\mathbb{Z} A_{\infty}^{\infty}$ and the $\mathbb{Z} D_{\infty}$ categories. In this section we define some Ext-finite noetherian hereditary abelian categories with almost split sequences and no non-zero projectives and injectives. Ultimately when we have our general classification result (Theorem IV.5.2) it will turn out that these are actually the same as the $\mathbb{Z} A_{\infty}^{\infty}$ and $\mathbb{Z} D_{\infty}$ categories. The constructions in this section are very easy, but unfortunately for one of them we have to assume that the characteristic is not two.

First construction. Following [30] we consider $R=k[u, v]$ as a $\mathbb{Z}^{2}$-graded ring with $\operatorname{deg} u=(1,0)$ and $\operatorname{deg} v=(0,1)$ and let $\mathcal{C}$ be the category of finitely generated graded $R$-modules modulo the finite dimensional ones. Then it is shown in [30] that $\mathcal{C}$ is a hereditary abelian category satisfying Serre duality (this last fact follows easily from graded local duality) and it is also Ext-finite. It is also shown in [30. that there are precisely two $\tau$-orbits of simples. Hence invoking Proposition 11 .3.4.4 above and Theorem IV.5.2 below it follows that $\mathcal{C}$ is the $\mathbb{Z} A_{\infty}^{\infty}$ category.

Second construction. Now we assume that the characteristic of $k$ is not two. Let $\mathcal{C}$ be as in the previous construction. The category $\mathcal{C}$ has a natural automorphism $\sigma$ of order two which sends a graded module $\left(M_{i j}\right)_{i j}$ to $\left(M_{j i}\right)_{i j}$ and which exchanges the $u$ and $v$ action. Let $\mathcal{D}$ be the category of $\mathbb{Z} / 2 \mathbb{Z}$ equivariant objects in $\mathcal{C}$, i.e. pairs $(M, \phi)$ where $\phi$ is an isomorphism $M \rightarrow \sigma(M)$ satisfying $\sigma(\phi) \phi=\mathrm{id}_{M}$. It now follows easily from the general results in [22] that $\mathcal{D}$ is an Ext-finite hereditary abelian category with almost split sequences and no projectives or injectives, whence $\mathcal{C}$ satisfies Serre duality by Theorem I.3.3 The two orbits of simples in $\mathcal{C}$ collapse to a single orbit in $\mathcal{D}$. Hence invoking Proposition $\amalg I$.3.5.2 above and Theorem IV.5.2 it follows $\mathcal{C}$ is the $\mathbb{Z} D_{\infty}$ category. 


\section{Hereditary nOETHERIAN ABELIAN CATEGORIES WITH NO PROJECTIVES OR INJECTIVES}

Let $\mathcal{C}$ be a connected $k$-linear Ext-finite noetherian hereditary abelian category having almost split sequences and no non-zero projectives or injectives, or equivalently, having a Serre functor and no non-zero projectives. In this section we show that if $\mathcal{C}$ has some object which is not of finite length, then $\mathcal{C}$ has one of the following forms:

1. If $\mathcal{C}$ has an infinite number of $\tau$-orbits of simples, then $\mathcal{C}$ is the category of coherent sheaves over a hereditary order over a connected non-singular proper curve (as in III.2).

2. If $\mathcal{C}$ has exactly two $\tau$-orbits of simples, then $\mathcal{C}$ is the $\mathbb{Z} A_{\infty}^{\infty}$ category (as in III.3.4).

3. If $\mathcal{C}$ has exactly one $\tau$-orbit of simples, then $\mathcal{C}$ is the $\mathbb{Z} D_{\infty}$ category (as in III.3.5).

We first show that when $\mathcal{C}$ is connected and satisfies the above hypothesis, then either each $\tau$-orbit of simple objects is finite or each such orbit is infinite. In the first case we show that there is a unique simple object in the quotient category $\mathcal{C} / \mathcal{T}$, where $\mathcal{T}$ consists of the objects of finite length, coming from an object $F$ in $\mathcal{C}$ with no summands in $\mathcal{T}$. We are able to construct an automorphism $t: \mathcal{C} \rightarrow \mathcal{C}$ such that $(F, t)$ is ample, and then we use results of [3] to show that $\mathcal{C}$ is equivalent to some quotient category qgr $S$. Then $\mathcal{C}$ is equivalent to the category of coherent sheaves over a hereditary order over a connected non-singular proper curve. In the second case we show that $\mathcal{C}$ is the $\mathbb{Z} A_{\infty}^{\infty}$ category if there is one simple object in $\mathcal{C} / \mathcal{T}$, and the $\mathbb{Z} D_{\infty}$ category if there are two simple objects.

In this section $\mathcal{C}$ will satisfy the above assumptions, except that we will not a priori assume that $\mathcal{C}$ is connected. As usual we denote the Serre functor by $F$ and we write $\tilde{\tau}=F[-1]$. The induced autoequivalence of $\mathcal{C}$ is as usual denoted by $\tau$.

IV.1. Preliminaries. In this section we show that if $\mathcal{C}$ is connected, then the quotient category $\mathcal{C} / \mathcal{T}$ (where $\mathcal{T}$ consists of the objects of finite length) contains at most two simple objects, and if there are two, then they are permuted by $\tau$ modulo $\mathcal{T}$. We also show that if $A$ is not in $\mathcal{T}$, there is some non-zero map from $A$ to a simple object in every $\tau$-orbit.

We call the objects in $\mathcal{T}$ "torsion". Analogously we call an object in $\mathcal{C}$ torsion free if it contains no subobject in $\mathcal{T}$. Let $\mathcal{F}$ be the full subcategory of $\mathcal{C}$ consisting of torsion free objects. Clearly $\mathcal{T}$ and $\mathcal{F}$ are stable under $\tau$ (as they are under any autoequivalence). In the sequel we denote the quotient functor $\mathcal{C} \rightarrow \mathcal{C} / \mathcal{T}$ by $\pi$.

Let $\left(S_{i}\right)_{i \in I}$ be the simple objects in $\mathcal{C}$, and let $v$ be the permutation of $I$ defined by $\tau S_{i} \cong S_{v(i)}$. We say that $i \sim j$ if $i$ and $j$ are in the same $v$-orbit. If $J \subset I$, then we denote by $\mathcal{T}_{J}$ the full subcategory of $\mathcal{C}$ consisting of objects whose Jordan-Hölder quotients are in $\left(S_{j}\right)_{j \in J}$. We have

$$
\mathcal{T}=\bigoplus_{o \in I / \sim} \mathcal{T}_{o}
$$

where the $\mathcal{T}_{o}$ are described by the results in III.1 
We will now prove some elementary properties of torsion free objects. Note first that by Serre duality $\operatorname{Ext}^{1}(\mathcal{F}, \mathcal{T})=0$. In other words the torsion free objects behave as projectives with respect to the torsion objects. From this we easily deduce:

Lemma IV.1.1. $\quad$ 1. Every object in $\mathcal{C}$ is the direct sum of a torsion object and a torsion free object.

2. The categories $\mathcal{T}_{o}$ for $o \in I / \sim$ are stable under essential extensions in $\mathcal{C}$.

If $J \subset I$ is $v$-stable and $A \in \mathcal{C}$, then we define the $\mathcal{T}_{J}$-topology on $A$ as the linear topology generated by the subobjects $A^{\prime} \subset A$ which satisfy $A / A^{\prime} \in \mathcal{T}_{J}$. An equivalent way of stating Lemma IV.1.1,2 is the following.

Lemma IV.1.2. The $\mathcal{T}_{J}$-topology satisfies the Artin-Rees condition.

Recall that the Artin-Rees condition means that if $A \subset B \subset D$ with $B / A \in \mathcal{T}_{J}$, then there exists $E \subset D$ with $D / E \in \mathcal{T}_{J}$ and $E \cap B \subset A$.

The following technical result will be used repeatedly.

Lemma IV.1.3. Let $A, D \in \mathcal{C}$ and let $\left(D_{i}\right)_{i \in I}$ be an inverse system of subobjects of $D$ which has the property that $\bigcap_{i} D_{i}=0$. Then there exists $i \in I$ such that for all $H \subset D_{i}$ we have $\operatorname{Ext}^{1}(H, A)=0$.

Proof. By Serre duality we have $\operatorname{Ext}^{1}\left(D_{i}, A\right) \cong \operatorname{Hom}\left(E, D_{i}\right)^{*}$ with $E=\tau^{-1} A$. We will show that $\operatorname{Hom}\left(E, D_{i}\right)=0$ for some $i \in I$. By the vanishing of $\operatorname{Ext}^{2}$ this automatically implies that $\operatorname{Ext}^{1}(H, A)=0$ for all $H \subset D_{i}$. Since $\operatorname{Hom}\left(E, D_{j}\right) \subset$ $\operatorname{Hom}\left(E, D_{i}\right)$ if $D_{j} \subset D_{i}$ and $\operatorname{Hom}\left(E, D_{i}\right)$ is finite dimensional, it suffices to show that this inclusion is not equality for small $D_{j}$ if $\operatorname{Hom}\left(E, D_{i}\right) \neq 0$. Let $f: E \rightarrow D_{i}$ be a non-zero map. Since $\bigcap_{i} D_{i}=0$, there must exist some $l$ such that im $f \not \subset D_{l}$ and hence some $D_{j} \subset D_{i} \cap D_{l}$ such that im $f \not \subset D_{j}$. Thus $f \notin \operatorname{Hom}\left(E, D_{j}\right)$ and we are done.

We have the following consequence.

Corollary IV.1.4. The category $\mathcal{C} / \mathcal{T}$ is semisimple.

Proof. It is sufficient to show that for any $A, D \in \mathcal{C}$ there is a subobject $D^{\prime} \subset D$ with $D / D^{\prime} \in \mathcal{T}$ such that $\operatorname{Ext}^{1}\left(D^{\prime}, A\right)=0$. To prove this we verify the hypotheses for Lemma IV.1.3 for the inverse system of cotorsion subobjects of $D$, that is, subobjects $D^{\prime}$ of $D$ with $D / D^{\prime} \in \mathcal{T}$. So let $E$ be a maximal subobject contained in all $D^{\prime} \subset D$ with $D / D^{\prime} \in \mathcal{T}$. If $E \neq 0$, then let $E^{\prime}$ be a maximal subobject of $E$. Since $E / E^{\prime}$ is simple we have $E / E^{\prime} \in \mathcal{T}$. By Artin-Rees we obtain the existence of $D^{\prime \prime} \subset D$ such that $D / D^{\prime \prime} \in \mathcal{T}$ and $D^{\prime \prime} \cap E \subset E^{\prime}$. This contradicts the definition of $E$. Hence $E=0$ and we are done.

The following easy lemma is used several times.

Lemma IV.1.5. 1. Assume that $F \in \mathcal{F}$ is simple modulo $\mathcal{T}$. Then $\operatorname{Hom}(F, F)$ $\cong k$.

2. Assume that $F, G \in \mathcal{F}$ are simple modulo $\mathcal{T}$. If $\operatorname{Hom}(F, G)$ and $\operatorname{Hom}(G, F)$ are both non-zero, then any non-trivial map $\phi: F \rightarrow G$ is an isomorphism.

Proof. 1. From the fact that $F$ is torsion free we obtain

$$
\operatorname{Hom}(F, F) \hookrightarrow \operatorname{Hom}_{\mathcal{C} / \mathcal{T}}(\pi F, \pi F)
$$


and since $F$ is simple modulo $\mathcal{T}$, the latter is a division algebra. So $\operatorname{Hom}(F, F)$ has no zero divisors. Since by our general hypotheses $\operatorname{Hom}(F, F)$ is finite dimensional, we obtain that $\operatorname{Hom}(F, F) \cong k$.

2. Pick non-trivial maps $\theta: G \rightarrow F, \phi: F \rightarrow G$. Then $\phi$ and $\theta$ represent nonzero maps in $\operatorname{Hom}_{\mathcal{C} / \mathcal{T}}(\pi F, \pi G)$ and $\operatorname{Hom}_{\mathcal{C} / \mathcal{T}}(\pi G, \pi F)$. Since $\pi F$ and $\pi G$ are simple, these maps must be isomorphisms. In particular the compositions $\phi \theta$ and $\theta \phi$ are non-zero in $\operatorname{Hom}_{\mathcal{C} / \mathcal{T}}(\pi F, \pi F)$ and $\operatorname{Hom}_{\mathcal{C} / \mathcal{T}}(\pi G, \pi G)$, and hence they are also non-zero in $\operatorname{Hom}(F, F)$ and $\operatorname{Hom}(G, G)$. It follows from 1. that $\phi \theta$ and $\theta \phi$ are scalar multiples of the identity map. Thus $\phi$ is an isomorphism.

Next we examine more closely the relation between the category $\mathcal{T}$ and the semisimple category $\mathcal{C} / \mathcal{T}$.

If $D \in \mathcal{C}$ is torsion free and $o$ is a $v$-orbit in $I$, then we say that $o$ is linked to $D$ if and only if there exists $i \in o$ together with a surjective map $D \rightarrow S_{i}$.

Lemma IV.1.6. $\quad$ 1. If $D$ is linked to o and if $D^{\prime}$ is isomorphic to $D$ modulo $\mathcal{T}$, then $D^{\prime}$ is also linked to $o$.

2. If $D$ is linked to o and $i \in o$, then there exists a torsion free object $D^{\prime}$ isomorphic to $D$ modulo $\mathcal{T}_{o}$ such that there is a surjective map $D^{\prime} \rightarrow S_{i}$.

Proof. Assume that there is a surjective map $D \rightarrow S_{i}$ where $i \in o$.

1. We can reduce to two cases.

(a) $D \subset D^{\prime}$ and $D^{\prime} / D=S_{p}$ for some simple object $S_{p}$. Applying $\operatorname{Hom}\left(-, S_{j}\right)$ to the short exact sequence

$$
0 \rightarrow D \rightarrow D^{\prime} \rightarrow S_{p} \rightarrow 0
$$

yields

$0 \rightarrow \operatorname{Hom}\left(S_{p}, S_{j}\right) \rightarrow \operatorname{Hom}\left(D^{\prime}, S_{j}\right) \rightarrow \operatorname{Hom}\left(D, S_{j}\right) \rightarrow \operatorname{Ext}^{1}\left(S_{p}, S_{j}\right) \rightarrow 0$

since $\operatorname{Ext}^{1}\left(D^{\prime}, S_{j}\right)=0$ using Serre duality. If $v(p) \neq i$, then $\operatorname{Ext}^{1}\left(S_{p}, S_{i}\right)$ $=0$ and hence $\operatorname{Hom}\left(D^{\prime}, S_{i}\right) \neq 0$ since $\operatorname{Hom}\left(D, S_{i}\right) \neq 0$. If $v(p)=i$, then $p \in o$, and we have $\operatorname{Hom}\left(D^{\prime}, S_{p}\right) \neq 0$.

(b) $D^{\prime} \subset D$ and $D / D^{\prime}=S_{p}$. Applying $\operatorname{Hom}\left(-, S_{j}\right)$ to the short exact sequence

$$
0 \rightarrow D^{\prime} \rightarrow D \rightarrow S_{p} \rightarrow 0
$$

yields

$0 \rightarrow \operatorname{Hom}\left(S_{p}, S_{j}\right) \rightarrow \operatorname{Hom}\left(D, S_{j}\right) \rightarrow \operatorname{Hom}\left(D^{\prime}, S_{j}\right) \rightarrow \operatorname{Ext}^{1}\left(S_{p}, S_{j}\right) \rightarrow 0$.

If $p \neq i$, then we take $j=i$ and we have $\operatorname{Hom}\left(S_{p}, S_{j}\right)=0$. If $p=i$, then we take $j=v(i)$ and we have $\operatorname{Ext}^{1}\left(S_{p}, S_{j}\right) \neq 0$. In both cases we find $\operatorname{Hom}\left(D^{\prime}, S_{j}\right) \neq 0$.

2. Assume that there is a non-zero map $\phi: D \rightarrow S_{j}$ (with $j \in o$ ). If $D^{\prime}=\operatorname{ker} \phi$, then it follows as above that $D^{\prime}$ maps non-trivially to $S_{v(j)}$. By Serre duality we have that $\operatorname{Ext}^{1}\left(S_{v^{-1}(j)}, D\right) \neq 0$. Let $D^{\prime \prime}$ be a non-trivial extension of $D$ and $S_{v^{-1}(j)}$. Then $D^{\prime \prime}$ is torsion free and maps non-trivially to $S_{v^{-1}(j)}$.

From the previous lemma we can now deduce the following perhaps slightly surprising result. 
Lemma IV.1.7. 1. The operation $\tau^{2}$ is the identity on objects in $\mathcal{C} / \mathcal{T}$.

2. If $D, D^{\prime} \in \mathcal{F}$ and $D^{\prime}=\tau D$ modulo $\mathcal{T}$, then $D$ and $D^{\prime}$ are linked to the same $o \in \mathcal{T} / \sim$.

3. If $D, D^{\prime} \in \mathcal{F}$ are simple modulo $\mathcal{T}$, then $D^{\prime}=D$ or $D^{\prime}=\tau D$ modulo $\mathcal{T}$ if and only if $D$ and $D^{\prime}$ are linked to a common $o \in \mathcal{T} / \sim$.

Proof. Clearly 2. and one direction of 3 . are trivial. We start by proving the nontrivial direction of 3 . So assume that $D$ and $D^{\prime}$ in $\mathcal{F}$ are simple modulo $\mathcal{T}$ and are linked to a common $o \in I / \sim$. By Lemma IV.1.6 we may assume that there exists $i \in o$ together with surjective maps $\phi: D \rightarrow S_{i}$ and $\phi^{\prime}: D^{\prime} \rightarrow S_{i}$. Let $E$ be the pullback of these maps and let $A$ be the kernel of $\phi^{\prime}$. Thus we have a commutative diagram with exact rows:

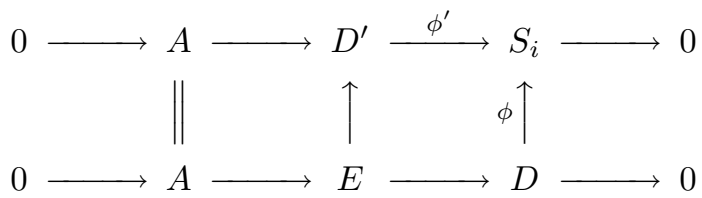

If the lower exact sequence splits, then $\phi$ factors through $\phi^{\prime}$. In particular there is a non-trivial map $\theta: D \rightarrow D^{\prime}$. Since $D^{\prime}$ and $D$ are simple modulo $\mathcal{T}$, it follows that $\theta$ must be an isomorphism modulo $\mathcal{T}$ since it is clearly not zero.

Assume now that the lower exact sequence in (IV.1.2) does not split. Then it yields a non-trivial element of $\operatorname{Ext}^{1}(D, A)$. Hence by Serre duality $\operatorname{Hom}\left(\tau^{-1} A, D\right) \neq$ 0 . Since $A=D^{\prime}$ modulo $\mathcal{T}$, we obtain $\tau^{-1} D^{\prime}=D$ modulo $\mathcal{T}$. This proves what we want.

Now 1. follows easily from 3. For let $D \in \mathcal{F}$ be simple modulo $\mathcal{T}$. Then $\tau^{2} D$ is linked to the same $o \in I / \sim$ as $D$. Hence by 3 . we have that $\tau^{2} D=D$ or $\tau^{2} D=\tau D$ modulo $\mathcal{T}$. Thus we find that in any case $\tau^{2} D=D$ modulo $\mathcal{T}$. This holds for all simples in $\mathcal{C} / \mathcal{T}$, and since this category is semisimple, it follows that $\tau^{2}$ is the identity on objects.

Corollary IV.1.8. Assume that $\mathcal{C}$ is connected. Then $\mathcal{C} / \mathcal{T}$ contains at most two simple objects, and if there are two they are permuted by $\tau$ modulo $\mathcal{T}$. Furthermore every $A \in \mathcal{F}$ is linked to every $o \in I / \sim$.

Proof. We first claim that every $o \in I / \sim$ is linked to at least one object in $\mathcal{F}$. This follows from the fact that $\operatorname{Hom}\left(\mathcal{T}_{o}, \mathcal{T}_{o^{\prime}}\right)=\operatorname{Hom}\left(\mathcal{T}_{o^{\prime}}, \mathcal{T}_{o}\right)=\operatorname{Hom}\left(\mathcal{T}_{o}, \mathcal{F}\right)=0$ for $o^{\prime} \neq o$. So if $\operatorname{Hom}\left(\mathcal{F}, \mathcal{T}_{o}\right)=0$, then $\mathcal{T}_{o}$ splits off as a factor from $\mathcal{C}$, contradicting the connectedness of $\mathcal{C}$.

Let the simple objects in $\mathcal{C} / \mathcal{T}$ be represented by $F_{1}, \ldots, F_{l} \in \mathcal{F}$. Assume that $F_{1}, \ldots, F_{t}$ constitute one $\tau$-orbit modulo $\mathcal{T}$ (thus $t=1,2$ ). Let $J, K$ be respectively the union of the orbits which are linked to $F_{1} \oplus \cdots \oplus F_{t}$ and $F_{t+1} \oplus \cdots \oplus F_{l}$. By Lemma IV.1.7 3 we have $J \cap K=\emptyset$ and by the previous paragraph $J \cup K=I$.

Define $\mathcal{C}_{1}$ as the full subcategory of $\mathcal{C}$ consisting of objects of the form $F \oplus T$ where $F$ is isomorphic to $F_{1}^{a_{1}} \oplus \cdots \oplus F_{t}^{a_{t}}$ modulo $\mathcal{T}_{J}$ and $T \in \mathcal{T}_{J}$. We define $\mathcal{C}_{2}$ in a similar way but using $F_{t+1}, \ldots, F_{l}$ and $K$. It is easy to see that $\mathcal{C}=\mathcal{C}_{1} \oplus \mathcal{C}_{2}$. This contradicts again the hypothesis that $\mathcal{C}$ is connected.

So we obtain that $l=t \leq 2$. Since every $o \in I / \sim$ is linked to at least one $F_{i}$, and since $\tau$ acts transitively, we obtain that every $o \in I / \sim$ is linked to every $F_{i}$. 
We will use the following.

Lemma IV.1.9. Assume that $\mathcal{C}$ is connected. Let $F \in \mathcal{F}$ and $o \in I / \sim$. Then the $\mathcal{T}_{o}$ topology on $F$ is separated.

Proof. This follows from the Artin-Rees property together with LemmaIV.1.6. See the proof of Corollary IV.1.4.

IV.2. Completion. In this section we use the results from $\S I V .1$ to show that if $\mathcal{C}$ is connected, then the orbits of the simple objects are either all finite or they are all infinite, and in the first case there is a unique simple object in $\mathcal{C} / \mathcal{T}$. To accomplish this, we use, as otherwise in this paper, some arguments inspired by geometry. For example we want to understand the formal local structure of the objects in $\mathcal{C}$ around a $\tau$-orbit of simples. For this we use the notion of "completion" which was introduced in 32 .

Let $o \in I / \sim$. By Appendix A $\mathcal{T}_{o}$ is a connected hereditary abelian category with all objects of finite length. Furthermore it is clear that Hom and $\operatorname{Ext}^{1}$ in $\mathcal{C}$ and $\mathcal{T}_{o}$ coincide. Thus $\mathcal{T}_{o}$ has a Serre functor and we can apply the results in to it. We will denote by $C_{o}$ the pseudo-compact ring which was denoted by $C$ in §II.1. We consider the elements of $C_{o}$ as matrices whose entries are indexed by the elements of $o$. For $D \in \mathcal{C}$ we define

$$
\hat{D}_{o}=\underset{D / D^{\prime} \in \mathcal{T}_{o}}{\operatorname{proj} \lim }\left(D / D^{\prime}\right)^{\wedge}
$$

where $(\hat{-})$ was defined in $\$$ III.1 If $o$ is clear from the context, then we write $(\hat{-})$ for $(\hat{-})_{0}$.

Clearly $(\hat{-})$ is a functor from $\mathcal{C}$ to $\mathrm{PC}\left(C_{o}\right)$. As in 32 one proves the following.

Lemma IV.2.1. $\quad$ 1. $(\hat{-})$ is an exact functor. In particular we can extend $(\hat{-})$ to a functor $D^{b}(\mathcal{C}) \rightarrow D^{b}\left(\mathrm{PC}\left(C_{o}\right)\right)$. This allows us to define the action of $(\hat{-})$ on Ext's.

2. If we restrict $(\hat{-})$ to $\mathcal{T}$, then it is the composition of the projection $\mathcal{T} \rightarrow \mathcal{T}_{o}$

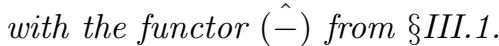

3. If we restrict $(\hat{-})$ to $\mathcal{F}$, then its image consists of projective pseudo-compact $C_{o}$-modules containing every indecomposable projective pseudo-compact $C_{o^{-}}$ module at most a finite number of times as a factor.

4. Let $D \in \mathcal{C}$ and $T \in \mathcal{T}_{o}$. Then completion defines an isomorphism

$$
\operatorname{Ext}_{\mathcal{C}}^{i}(D, T) \cong \operatorname{Ext}_{\mathrm{PC}\left(C_{o}\right)}^{i}(\hat{D}, \hat{T}) .
$$

5. For every $D \in \mathcal{C}$ we have a natural isomorphism

$$
\widehat{\tau D} \cong \hat{D}_{o} \otimes_{C_{o}} \omega_{C_{o}}
$$

Proof. This theorem is proved in the same way as 32, Thm. 5.3.1, Prop. 5.3.4, Cor. 5.3.5]. The only thing that needs to be proved slightly differently is 3 .

So let $D \in \mathcal{F}$. To prove that $\hat{D}$ is projective we take a minimal projective resolution

$$
0 \rightarrow P \rightarrow Q \rightarrow \hat{D} \rightarrow 0
$$

By the definition of a minimal resolution we have $P \subset \operatorname{rad} Q$. This yields for $j \in o$ : $\operatorname{Hom}_{\mathrm{PC}\left(C_{o}\right)}\left(P, \hat{S}_{j}\right) \cong \operatorname{Ext}^{1}\left(\hat{D}, \hat{S}_{j}\right) \cong \operatorname{Ext}^{1}\left(D, S_{j}\right)=0$. Thus $P=0$. 
Let $M$ be indecomposable projective over $C_{o}$. Then $M / \operatorname{rad}(M) \cong \hat{S}_{j}$ for some $j$ in $o$. The number of times that $M$ occurs in a direct sum decomposition of $\hat{D}$ is given by the dimension of $\operatorname{Hom}_{\mathrm{PC}\left(C_{o}\right)}\left(\hat{D}, \hat{S}_{j}\right) \cong \operatorname{Hom}_{\mathcal{C}}\left(D, S_{j}\right)$, which is finite by hypothesis.

Our next aim is to sharpen Lemma IV.2.1 by actually showing that $\hat{D}$ is a noetherian $C_{o}$-module. We start by proving the following result.

Lemma IV.2.2. Let $D \in \mathcal{C}$ and $o \in I / \sim$. Then there exist only a finite number of $j \in$ o such that there is a non-zero map $D \rightarrow S_{j}$.

Proof. Assume that the lemma is false. So $D$ maps to an infinite number of different $S_{j}$ with $j \in o$. In particular o must be infinite. Without loss of generality we may assume that $D$ is torsion free and that $D$ has simple image in $\mathcal{C} / \mathcal{T}$.

Choose an element $i \in o$ and write $P_{l}=e_{v^{l}(i)} C_{o}$ where for $j \in o$ the corresponding diagonal idempotent in $C_{o}$ is denoted by $e_{j}$. Thus by the explicit structure of $C_{o}$ given in

$$
\operatorname{Hom}\left(P_{l}, P_{l^{\prime}}\right)= \begin{cases}0 & \text { if } l<l^{\prime} \\ k & \text { otherwise. }\end{cases}
$$

Since by Lemma IV.2.1 it follows that $\hat{D}$ is projective with finite multiplicities, we have

$$
\hat{D}=\prod_{l \in \mathbb{Z}} P_{l}^{a_{l}}
$$

for certain $l \in \mathbb{Z}$. By Proposition $\amalg I I .1 .2$ we obtain

$$
\widehat{\tau^{-1} D}=\prod_{l \in \mathbb{Z}} P_{l-1}^{a_{l}} .
$$

Define $\tau_{l, m}$ as $P_{l} / \operatorname{rad}^{m}\left(P_{l}\right)$ and let $T_{l, m}$ be the object in $\mathcal{T}_{o}$ with the property that $\hat{T}_{l, m}=\tau_{l, m}$. Thus $T_{l, m}$ is the unique object of length $m$ with cosocle $S_{v^{l}(i)}$. We have

$$
\operatorname{Hom}\left(P_{l}, \tau_{l^{\prime}, m}\right)= \begin{cases}k & \text { if } l^{\prime} \leq l \leq l^{\prime}+m-1 \\ 0 & \text { otherwise }\end{cases}
$$

Put

$$
T_{n}=T_{n, 1}^{a_{n}} \oplus T_{n-1,2}^{a_{n-1}} \oplus \cdots \oplus T_{-n, 2 n+1}^{a_{-n}}
$$

and $\tau_{n}=\hat{T}_{n}$. Then combining the canonical surjective maps $P_{l} \rightarrow \tau_{l, m}$ yields a canonical surjective map $\hat{D} \rightarrow \tau_{n}$ and hence by Lemma IV.2.1 a surjective map $D \rightarrow T_{n}$.

Let $A_{n}$ be the kernel of this map. Using the exactness of completion we find

$$
\hat{A}_{n}=\prod_{l<-n} P_{l}^{a_{l}} \oplus P_{n+1}^{a_{-n}+\cdots+a_{n}} \oplus \prod_{l>n} P_{l}^{a_{l}} .
$$

Applying $\operatorname{Hom}(-, D)$ to the exact sequence

$$
0 \rightarrow A_{n} \rightarrow D \rightarrow T_{n} \rightarrow 0
$$

we obtain the exact sequence

$$
0 \rightarrow \operatorname{Hom}(D, D) \rightarrow \operatorname{Hom}\left(A_{n}, D\right) \rightarrow \operatorname{Ext}^{1}\left(T_{n}, D\right) \rightarrow \operatorname{Ext}^{1}(D, D)
$$


Furthermore $\operatorname{dim} \operatorname{Ext}^{1}\left(T_{n}, D\right)=\operatorname{dim} \operatorname{Hom}\left(\tau^{-1} D, T_{n}\right)=\operatorname{dim} \operatorname{Hom}\left(\widehat{\tau^{-1} D}, \tau_{n}\right)$. This goes to infinity by (IV.2.2), (IV.2.3) and the definition of $\tau_{n}$. It follows that $\operatorname{dim} \operatorname{Hom}\left(A_{n}, D\right)$ also goes to infinity. On the other hand we now show that in fact $\operatorname{Hom}\left(A_{n}, D\right) \cong k$. This is clearly a contradiction.

We apply $\operatorname{Hom}\left(A_{n},-\right)$ to the exact sequence (IV.2.5). This yields the exact sequence

$$
0 \rightarrow \operatorname{Hom}\left(A_{n}, A_{n}\right) \rightarrow \operatorname{Hom}\left(A_{n}, D\right) \rightarrow \operatorname{Hom}\left(A_{n}, T_{n}\right) .
$$

From the fact that $A_{n}$ is simple modulo $\mathcal{T}$ we obtain that $\operatorname{Hom}\left(A_{n}, A_{n}\right) \cong k$ by Lemma IV.1.5

On the other hand, by completing and using (IV.2.4 $)$ and (IV.2.3) we find $\operatorname{Hom}\left(A_{n}, T_{n}\right)=0$. This proves what we want.

Corollary IV.2.3. Denote by $\mathrm{pc}\left(C_{o}\right)$ the full subcategory of $\mathrm{PC}\left(C_{o}\right)$ consisting of noetherian objects. Then $(-)_{o}$ defines a functor $\mathcal{C} \rightarrow \operatorname{pc}\left(C_{o}\right)$.

Proof. It clearly suffices to show that if $F \in \mathcal{F}$, then $\hat{F}$ is noetherian. Now we know that $\hat{F}$ is a projective object in $\operatorname{PC}\left(C_{o}\right)$ mapping only to a finite number of different simples (counting multiplicities). Hence $\hat{F}$ is a direct sum of a finite number of indecomposable projectives. Since the indecomposable pseudo-compact projectives over $C_{o}$ are noetherian, it follows that $\hat{F}$ is noetherian.

Note that if $|o|=\infty$, then $\operatorname{pc}\left(C_{o}\right)$ is equivalent to $\operatorname{gr}(R)$ with $R=k[x]$.

Corollary IV.2.4. Let $D \in \mathcal{C}$ and $T \in \mathcal{T}_{o}$. Then completion defines an isomorphism

$$
\operatorname{Ext}_{\mathcal{C}}^{i}(T, D) \rightarrow \operatorname{Ext}_{\mathrm{PC}\left(C_{o}\right)}^{i}(\hat{T}, \hat{D}) .
$$

Proof. Let $\eta_{T}: \operatorname{Ext}^{1}(T, \tau T) \rightarrow k$ be the linear map corresponding to the identity map $T \rightarrow T$ under Serre duality. As in the proof of Proposition [.2.3 it follows that $\eta_{T}$ can be chosen freely, subject to the condition that it must be non-vanishing on the almost split sequence ending in $T$.

Now define $\eta_{\hat{T}}: \operatorname{Ext}^{1}(\hat{T}, \tau \hat{T}) \rightarrow k$ as $\hat{\eta}_{T}$. Then by functoriality we have a commutative diagram:

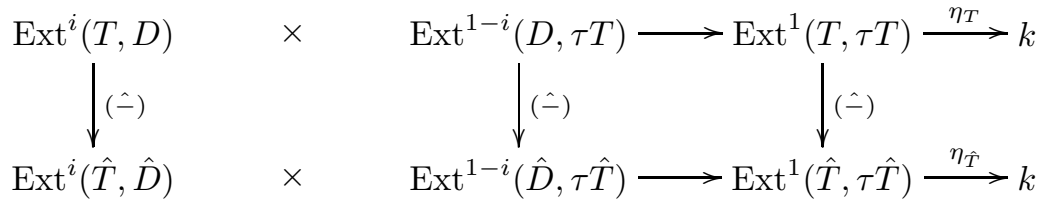

It follows that the pairings for $\mathcal{C}$ and for $\operatorname{PC}\left(C_{o}\right)$ are compatible. The upper pairing is non-degenerate by Serre duality and the lower pairing is non-degenerate by local duality for $C_{o}$ (or graded local duality for $k[x]$ if $|o|=\infty$ ). Using these dualities (IV.2.6) follows from (IV.2.1).

Let $\operatorname{dis}\left(C_{o}\right)$ be the pseudo-compact finite length modules over $C_{o}$ and put $\operatorname{qpc}\left(C_{o}\right)$ $=\operatorname{pc}\left(C_{o}\right) / \operatorname{dis}\left(C_{o}\right)$. Using Morita theory we obtain

$$
\operatorname{qpc}\left(C_{o}\right) \cong \begin{cases}\bmod \left(\left(C_{o}\right)_{x}\right) \cong \bmod k((x)) & \text { if }|o|<\infty \\ \operatorname{gr}\left(k\left[x, x^{-1}\right]\right) \cong \bmod (k) & \text { if }|o|=\infty\end{cases}
$$


Then completion defines an exact functor $\mathcal{C} / \mathcal{T}_{O} \rightarrow \operatorname{qpc}\left(C_{o}\right)$. So we obtain the commutative diagram

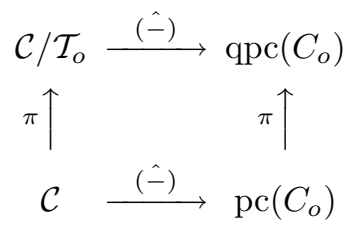

where as usual $\pi$ denotes the quotient functor. In the next section we will show that this is actually a pullback diagram.

Lemma IV.2.5. Let $D, E \in \mathcal{C}$ and assume that $o \in I / \sim$ is such that the linear topology on $E$ defined by $\mathcal{T}_{o}$ is separated. Then the canonical map

$$
\operatorname{Hom}_{\mathcal{C}}(D, E) \rightarrow \operatorname{Hom}_{\mathrm{PC}\left(C_{o}\right)}(\hat{D}, \hat{E})
$$

is injective.

Proof. This is trivial.

Corollary IV.2.6. Assume that $F \in \mathcal{F}$ is simple modulo $\mathcal{T}$. Assume furthermore that $F$ is linked to an infinite orbit $o \in I / \sim$. Then $\operatorname{Hom}_{\mathcal{C} / \mathcal{T}}(\pi F, \pi F) \cong k$.

Proof. By hypotheses $\operatorname{Hom}_{\mathcal{C} / \mathcal{T}}(\pi F, \pi F)$ is a skew field. Since $k$ is algebraically closed, it now suffices to show that $\operatorname{Hom}_{\mathcal{C} / \mathcal{T}}(\pi F, \pi F)$ is finite dimensional.

We have

$$
\operatorname{Hom}_{\mathcal{C} / \mathcal{T}}(\pi F, \pi F)=\underset{F / F_{1} \in \mathcal{T}}{\lim _{\mathcal{T}}} \operatorname{Hom}_{\mathcal{C}}\left(F_{1}, F\right) .
$$

By Lemma IV.2.5 the $\mathcal{T}_{o}$ topology on $F_{1}$ and $F$ is separated. Hence

$$
\underset{F / F_{1} \in \mathcal{T}}{\underset{\lim }{\longrightarrow}} \operatorname{Hom}_{\mathcal{C}}\left(F_{1}, F\right) \subset \underset{F / F_{1} \in \mathcal{T}}{\underset{\lim }{\longrightarrow}} \operatorname{Hom}_{\mathrm{pc}\left(C_{o}\right)}\left(\hat{F}_{1}, \hat{F}\right) .
$$

If $F_{1}$ runs through the subobjects in $F$ with the property that $F / F_{1} \in \mathcal{T}$, then $\hat{F}_{1}$ runs through the subobjects $G$ of $\hat{F}$ such that $\hat{F} / G \in \operatorname{dis}\left(C_{o}\right)$. Thus

$$
\begin{aligned}
\underset{F / F_{1} \in \mathcal{T}}{\lim _{\vec{T}}} \operatorname{Hom}_{\mathrm{pc}\left(C_{o}\right)}\left(\hat{F}_{1}, \hat{F}\right) & =\underset{\substack{G \in \operatorname{pc}\left(C_{o}\right) \\
\hat{F} / G \in \operatorname{dis}\left(C_{o}\right)}}{\lim _{\mathrm{puc}\left(C_{o}\right)}(\pi \hat{F}, \pi \hat{F}) .} \\
= & \operatorname{Hom}_{\mathrm{pc}\left(C_{o}\right)}(G, \hat{F})
\end{aligned}
$$

By (IV.2.7) this is finite dimensional.

By contrast the situation in the case of linkage to a finite orbit is different.

Lemma IV.2.7. Assume that $F, F^{\prime} \in \mathcal{F}$ are simple modulo $\mathcal{T}$. Assume furthermore that $F$ and $F^{\prime}$ are linked to a finite orbit $o \in I / \sim$. Then $\operatorname{Hom}_{\mathcal{C} / \mathcal{T}}\left(\pi F, \pi F^{\prime}\right)$ is infinite dimensional.

Proof. Since $F$ and $F^{\prime}$ are torsion free one has that $\operatorname{Hom}_{\mathcal{C} / \mathcal{T}}\left(\pi F, \pi F^{\prime}\right)$ is equal to the union of $\operatorname{Hom}\left(F_{1}, F^{\prime}\right)$ where $F / F_{1} \in \mathcal{T}$. Hence it suffices to show that the dimension of $\operatorname{Hom}\left(F_{1}, F^{\prime}\right)$ goes to infinity if $F_{1}$ runs through the subobjects of $F$ satisfying $F / F_{1} \in \mathcal{T}_{o}$. Put $T=F / F_{1}$. Applying $\operatorname{Hom}\left(-, F^{\prime}\right)$ to the exact sequence

$$
0 \rightarrow F_{1} \rightarrow F \rightarrow T \rightarrow 0
$$


yields the exact sequence

$$
0 \rightarrow \operatorname{Hom}\left(F, F^{\prime}\right) \rightarrow \operatorname{Hom}\left(F_{1}, F^{\prime}\right) \rightarrow \operatorname{Ext}^{1}\left(T, F^{\prime}\right) \rightarrow \operatorname{Ext}^{1}\left(F, F^{\prime}\right) .
$$

Hence it suffices to show that the dimension of $\operatorname{Ext}^{1}\left(T, F^{\prime}\right)$ goes to infinity. By Serre duality and completion we must show that the dimension of $\operatorname{Hom}\left(\hat{F}^{\prime}, \widehat{\tau T}\right)$ goes to infinity. To this end it is sufficient to show that for indecomposable projectives $P$ and $P^{\prime}$ over $C_{o}$ the dimension of $\operatorname{Hom}_{C_{o}}\left(P^{\prime}, S \otimes \omega_{C_{o}}\right)$ goes to infinity where $S$ runs through the finite dimensional quotients of $P$. This is an easy direct verification using the explicit structure of $C_{o}$ given in

Corollary IV.2.8. Assume that $\mathcal{C}$ is connected. Then the $v$-orbits in $I$ are either all finite, or all infinite. Furthermore if they are finite, then there exists only one simple object in $\mathcal{C} / \mathcal{T}$.

Proof. Assume that there are both finite and infinite orbits. Then by Corollary IV.1.8 there exists $F \in \mathcal{F}$, simple in $\mathcal{C} / \mathcal{T}$, such that $F$ is linked to both a finite and an infinite orbit. This is a contradiction according to Corollary IV.2.6 and Lemma IV.2.7.

Assume now that the orbits are finite but that there exist objects $F, F^{\prime} \in \mathcal{F}$ which are distinct and simple modulo $\mathcal{T}$. Clearly $\operatorname{Hom}_{\mathcal{C} / \mathcal{T}}\left(\pi F, \pi F^{\prime}\right)=0$. On the other hand by Corollary IV.1.8 we know that $F$ and $F^{\prime}$ are both linked to the same finite orbit, whence by Lemma IV.2.7 it follows that $\operatorname{Hom}_{\mathcal{C} / \mathcal{T}}\left(\pi F, \pi F^{\prime}\right)$ is infinite dimensional. This is clearly a contradiction.

IV.3. Description in terms of a pullback diagram. In this section we give useful descriptions of $\mathcal{C}$ which allow us to construct autoequivalences of $\mathcal{C}$ with desired properties. This will be used for the construction of ample pair in the next section.

We start with the following description of $\mathcal{C}$.

Lemma IV.3.1. (IV.2.8) is a pullback diagram in the sense that $\mathcal{C}$ is equivalent to the category of triples $(F, Z, \phi)$ where $F \in \mathcal{C} / \mathcal{T}_{o}, Z \in \operatorname{pc}\left(C_{o}\right)$ and $\phi$ is an isomorphism $\hat{F} \rightarrow \pi Z$.

Proof. Let $\mathcal{C}^{\prime}$ be the category of triples defined in the statement of the lemma. Define $U: \mathcal{C} \rightarrow \mathcal{C}^{\prime}$ by $U F=(\pi F, \hat{F}, I)$ where $I$ is the natural isomorphism $\pi \hat{F} \rightarrow$ $(\pi F)^{\wedge}$. We have to show that $U$ is an equivalence. To do this we show that $U$ is respectively faithful, full and essentially surjective.

Faithfulness. Assume that $F_{1}, F_{2} \in \mathcal{C}$ and that $f: F_{1} \rightarrow F_{2}$ is a homomorphism such that $U f=0$. Thus $\pi f=0$ and $\hat{f}=0$. The fact that $\pi f=0$ means that $f$ factors as $F_{1} \stackrel{f_{1}}{\longrightarrow} T \stackrel{f_{2}}{\longrightarrow} F_{2}$ where $T \in \mathcal{T}_{o}$, the map $f_{1}$ is an epimorphism and $f_{2}$ is a monomorphism. Then $0=\hat{f}$ factors likewise. This then implies that $\hat{T}=0$ and hence $T=0$. We conclude $f=0$.

Fullness. Let $F_{1}$ and $F_{2}$ be as in the previous paragraph. Now assume that we are given maps $f^{\prime}: \pi F_{1} \rightarrow \pi F_{2}$ and $f^{\prime \prime}: \hat{F}_{1} \rightarrow \hat{F}_{2}$ such that $\hat{f}^{\prime}=\pi f^{\prime \prime}$. We have to produce a map $f: F_{1} \rightarrow F_{2}$ such that $f^{\prime}=\pi f$ and $f^{\prime \prime}=\hat{f}$. Let $g: G_{1} \rightarrow F_{2}$ be a representative of $f^{\prime}$ in $\mathcal{C}$ where $G_{1}$ is a subobject of $F_{1}$ such that $F_{1} / G_{1} \in \mathcal{T}_{o}$. Below we denote the inclusion map $G_{1} \rightarrow F_{1}$ by $\alpha$. 
We now have $\pi \hat{g}=\pi f^{\prime \prime}$. This means that there exist $G_{1}^{\prime} \subset \hat{G}_{1}$ with $\hat{G}_{1} / G_{1}^{\prime} \in$ $\operatorname{dis}\left(C_{o}\right)$ such that the compositions $G_{1}^{\prime} \rightarrow \hat{G}_{1} \stackrel{\hat{g}}{\rightarrow} \hat{F}_{2}$ and $G_{1}^{\prime} \rightarrow \hat{G}_{1} \rightarrow \hat{F}_{1} \stackrel{f^{\prime \prime}}{\rightarrow} \hat{F}_{2}$ are equal.

From (IV.2.1) it follows easily that there exists $K_{1}$ which is a subobject of $G_{1}$ with cokernel in $\mathcal{T}_{o}$ such that $\hat{K}_{1}=G_{1}^{\prime}$. We now replace $G_{1}$ by $K_{1}$, and $g$ by the composition $K_{1} \rightarrow G_{1} \stackrel{g}{\rightarrow} F_{2}$. Hence we obtain the following commutative diagram:

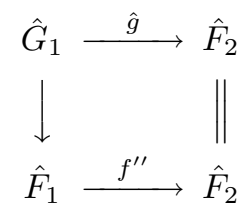

So our problem is now to lift $g$ to a homomorphism $f: F_{1} \rightarrow F_{2}$ such that $\hat{f}=f^{\prime \prime}$. Let $H$ be the pushout of $G_{1} \stackrel{g}{\rightarrow} F_{2}$ and $G_{1} \stackrel{\alpha}{\longrightarrow} F_{1}$. Then we have a commutative diagram with exact rows

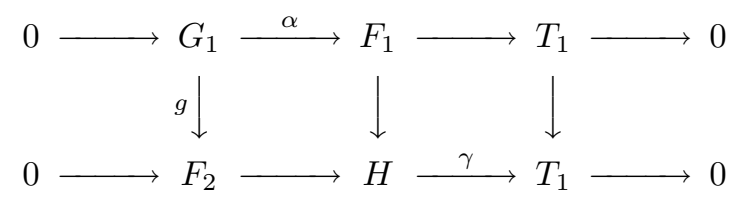

with $T_{1} \in \mathcal{T}_{o}$. It is easy to see that factorizations of $g$ through $\alpha$ are in oneone correspondence with splittings of $\gamma$. Similarly factorizations of $\hat{g}$ through $\hat{\alpha}$ correspond to splittings of $\hat{\gamma}$.

Now we know that $\hat{g}$ factors through $\hat{\alpha}$ and hence this yields a splitting of $\hat{\gamma}$. By CorollaryIV.2.4 and (IV.2.1) this splitting corresponds to a splitting of $\gamma$ and thus to a factorization $f$ of $g$ through $\alpha$. It is now easy to see that this is the $f$ we are looking for.

Essential surjectivity. Let $(F, Z, \phi) \in \mathcal{C}^{\prime}$. We have to show that this is in the essential image of $U$. Choose $G \in \mathcal{C}$ representing $F$. By definition $\phi$ is now an isomorphism $\pi \hat{G} \rightarrow \pi Z$.

As usual $\phi$ is represented by an injective map $\theta^{\prime}: K_{1}^{\prime} \rightarrow Z$ with cokernel in $\operatorname{dis}\left(C_{o}\right)$ where $K_{1}^{\prime}$ is a subobject of $\hat{G}$ also with cokernel in $\operatorname{dis}\left(C_{o}\right)$.

Thus we have the following maps:

$$
\hat{G} \leftarrow K_{1}^{\prime} \stackrel{\theta^{\prime}}{\longrightarrow} Z .
$$

By working from left to right and employing (IV.2.1) and (IV.2.6) these arrows and objects can be "uncompleted". That is, there are arrows and objects in $\mathcal{C}$

$$
G \leftarrow K_{1} \stackrel{\theta^{\prime \prime}}{\longrightarrow} L
$$

such that completion gives (IV.3.1).

Now $\hat{L}=Z$ and $\pi L \cong \pi G \cong F$. Checking the appropriate commutative diagrams we find that indeed $U L \cong(F, Z, \phi)$. 
We may use the previous lemma to define for any orbit $o$ in $I$ a canonical associated autoequivalence. Consider the following commutative diagram of functors:

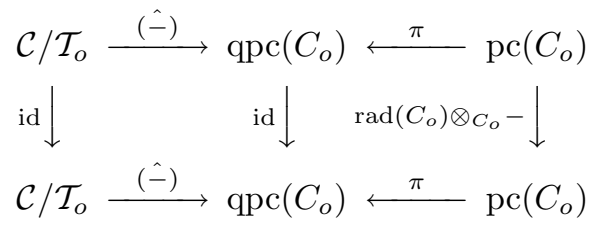

The vertical arrows are clearly autoequivalences, and hence, using Lemma IV.3.1 they define an autoequivalence on $\mathcal{C}$ which we will denote by $s_{o}$.

The functor $s_{o}$ has a more direct description on torsion free objects.

Lemma IV.3.2. Assume that $F \in \mathcal{F}$. Then $s_{o} F$ is the kernel of the universal map

$$
F \rightarrow \bigoplus_{i \in o} S_{i} \otimes \operatorname{Hom}\left(F, S_{i}\right)^{*}
$$

which in addition is surjective.

Proof. Surjectivity of (IV.3.3) follows from the fact that there are only a finite number of $i \in o$ such that $\operatorname{Hom}\left(F, S_{i}\right) \neq 0$ (see Lemma IV.2.2).

To prove that IV.3.3) gives the correct result for $s_{O}$ on $\mathcal{C}$ one has to check that it gives the correct result on $\mathcal{C} / \mathcal{T}_{o}, \operatorname{pc}\left(C_{o}\right)$ and $\operatorname{qpc}\left(C_{o}\right)$. In each of the cases this is clear.

From Proposition III.1.2 we also obtain the following obvious fact.

Lemma IV.3.3. The functor $s_{o}$ is (non-canonically) isomorphic to $\tau$ when restricted to $\mathcal{T}_{o}$. It is the identity on $\mathcal{T}_{o^{\prime}}$ for $o^{\prime} \neq o$. In particular

$$
s_{o}\left(S_{i}\right) \cong \begin{cases}S_{v(i)} & \text { if } i \in o, \\ S_{i} & \text { if } i \notin o .\end{cases}
$$

The foregoing results have an obvious extension in the case of a finite number of $v$ orbits $o_{1}, \ldots, o_{n}$. To state this let $J$ be the union of these orbits. Then there is a pullback diagram:

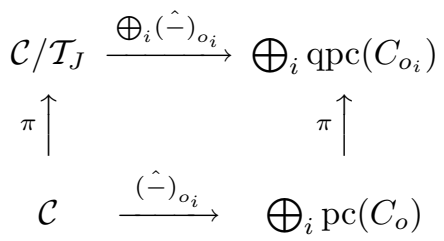

In the same way as for a single orbit we can define an associated autoequivalence $s_{J}$. It is easy to see that $s_{J}=s_{O_{1}} \cdots s_{o_{n}}$. Furthermore if $F \in \mathcal{F}$, then $s_{J}$ is given by the kernel of the surjective universal map

$$
F \rightarrow \bigoplus_{i \in J} S_{i} \otimes \operatorname{Hom}\left(F, S_{i}\right)^{*}
$$

Finally on simples we have:

$$
s_{J}\left(S_{i}\right) \cong \begin{cases}S_{v(i)} & \text { if } i \in J \\ S_{i} & \text { if } i \notin J\end{cases}
$$


IV.4. The finite orbit case. In this section we complete our classification in the finite orbit case, by constructing an ample pair $(F, t)$ and applying results from [3].

If $\mathcal{D}$ is a noetherian Ext-finite abelian category and $(F, t)$ is a pair consisting of an object $F \in \mathcal{D}$ and an autoequivalence $t$ of $\mathcal{D}$, then following [3] we will say that this pair is ample if the following conditions hold:

(a) For every $D \in \mathcal{D}$ there exists a surjective map $\bigoplus_{i=1}^{m} t^{-n_{i}} F \rightarrow D$ with $n_{i} \geq 0$.

(b) For every surjective map $E \rightarrow D$ in $\mathcal{D}$ the induced map $\operatorname{Hom}\left(t^{-n} F, E\right) \rightarrow$ $\operatorname{Hom}\left(t^{-n} F, D\right)$ is surjective for large $n$.

To a pair $(F, t)$ one can canonically associate a graded ring $\Gamma^{*}(F)$ defined by

$$
\Gamma^{*}(F)=\bigoplus_{n \in \mathbb{Z}} \operatorname{Hom}\left(t^{-n} F, F\right) .
$$

It is shown in 3] that if $(F, t)$ is ample, then $\Gamma^{*}(F)_{\geq 0}$ is a noetherian ring and $\mathcal{D}$ is equivalent to $\operatorname{qgr}\left(\Gamma^{*}(F)_{\geq 0}\right)$.

In this section we impose the usual hypotheses on $\mathcal{C}$, but we assume in addition that $\mathcal{C}$ is connected and that all $v$-orbits in $I$ are finite.

By Corollary IV.2.8 there is exactly one simple object modulo $\mathcal{T}$. Assume it is represented by $F \in \mathcal{F}$. Since $\tau F=F$ modulo $\mathcal{T}$, there must exist a finite number of orbits $o_{1}, \ldots, o_{n}$ in $I$ such that $\tau F=F$ modulo $\mathcal{T}_{J}$ with $J=o_{1} \cup \cdots \cup o_{n}$. Define $s_{J}$ as in Section IV.3. Writing $s=s_{J}$ and defining $t=s^{-1}$, we have the following.

Lemma IV.4.1. $(F, t)$ is an ample pair in $\mathcal{C}$.

Proof. The $\mathcal{T}_{J}$-topology on $F$ is separated by Lemma IV.1.9 So by Lemma IV.1.3 there exists a subobject $F_{1} \subset F$ with $F / F_{1} \in \mathcal{T}_{J}$ and with the property $\operatorname{Ext}^{1}\left(F^{\prime}, F\right)$ $=0$ for every subobject $F^{\prime}$ of $F_{1}$. By (IV.3.5) there exists an $n_{0}$ such that $s^{n} F \subset F_{1}$ for $n \geq n_{0}$. Hence we have

$$
\operatorname{Ext}^{1}\left(t^{-n} F, F\right)=\operatorname{Ext}^{1}\left(s^{n} F, F\right)=0
$$

for $n \gg 0$. From this we easily obtain that if we can show (a) then for all $X \in \mathcal{D}$ we have $\operatorname{Ext}^{1}\left(t^{-n} F, X\right)=0$ for $n \gg 0$. Thus (b) follows.

Now let $D \in \mathcal{C}$ and let $E$ be the largest subobject of $D$ which can be written as a quotient of a direct sum $\bigoplus_{i=1}^{t} s^{n_{i}} F$ with $n_{i} \geq 0$. Assume $D \neq E$ and write $T=D / E$. As above there exists $n_{0}$ such that $\operatorname{Ext}^{1}\left(s^{n} F, E\right)=0$ for $n \geq n_{0}$. On the other hand we claim that there exists a non-trivial map $s^{m} F \rightarrow T$ for some $m \geq n_{0}$. By the vanishing of $\operatorname{Ext}^{1}\left(s^{m} F, E\right)$ this map then lifts to a non-trivial map $s^{m} F \rightarrow D$, contradicting the choice of $E$.

It remains to show that there is a non-trivial map $s^{m} F \rightarrow T$ for some $m \geq n_{0}$. Assume first that $T$ contains a torsion free subobject $T^{\prime}$. We may assume that $T^{\prime}$ is simple modulo $\mathcal{T}$. Then the proof of Lemma IV.2.7 together with Corollary IV.1.8 shows that there is a non-trivial map $F^{\prime} \rightarrow T^{\prime}$ with $F / F^{\prime} \in \mathcal{T}_{o}$. As above we have that there is some $s^{n} F \subset F^{\prime}$. Since $F / s^{n} F$ is torsion, the composition $s^{n} F \rightarrow F^{\prime} \rightarrow T^{\prime}$ cannot be zero. This proves what we want.

Assume now that $T$ is torsion, let $S_{i}$ for $i \in I$ be a simple subobject of $T$, and let $o$ be the $v$-orbit of $i$. Then according to Lemma IV.1.6 there exists $j \in o$ together with a non-trivial map $F \rightarrow S_{j}$. We now consider two possibilities:

1. $o \not \subset J$. Since $F=\tau^{n} F$ modulo $\mathcal{T}_{J}$ for all $n$, it follows that there will also be a non-trivial map $\tau^{n} F \rightarrow S_{j}$ for all $n$, whence a non-trivial map $F \rightarrow S_{v^{-n}(j)}$. Choose $n$ in such a way that $v^{-n} j=i$ and apply $s^{m}$. This yields a non-trivial map $s^{m} F \rightarrow S_{i}$ for all $m$. 
2. $o \subset J$. Now we have a surjective map $s^{n} F \rightarrow S_{v^{n}(j)}$. Since the $v$-orbit of $j$ is finite, it follows that we can always find $m \geq n_{0}$ such that $s^{m} F$ maps surjectively to $S_{i}$.

Hence in both cases we obtain a non-trivial map $s^{m} F \rightarrow S_{i}$ with $m \geq n_{0}$. By the above discussion we are done.

What remains to be done in order to determine $\mathcal{C}$ explicitly is to compute $R=$ $\Gamma^{*}(F)_{\geq 0}$. We do this in a number of steps.

Step 1. $R$ is positively graded and $R_{0}=k$. Indeed from the fact that $F$ is simple modulo $\mathcal{T}$ it follows that $\operatorname{Hom}(F, F) \cong k$ (see Lemma IV.1.5).

Step 2. $R$ is a domain. This follows since $R_{n}$ embeds in $\operatorname{Hom}_{\mathcal{C} / \mathcal{T}}(\pi F, \pi F)$, and since $F$ is simple modulo $\mathcal{T}$, the latter is a division algebra $D$. The multiplication $R_{n} \otimes R_{m} \rightarrow R_{m+n}$ corresponds to multiplication in $D$. Hence we obtain an inclusion $R \hookrightarrow D\left[c, c^{-1}\right]$ where $c$ represents the inclusion $s F \rightarrow F$. Since $c$ also represents the identity element in $\operatorname{Hom}_{\mathcal{C} / \mathcal{T}}(\pi F, \pi F)$, we find in addition that $c$ is a central non-zero divisor in $R$.

Step 3. $R$ has Gelfand-Kirillov dimension two. To see this apply $\operatorname{Hom}(-, F)$ to the exact sequence

$$
0 \rightarrow s^{n+1} F \rightarrow s^{n} F \rightarrow s^{n}(F / s F) \rightarrow 0 .
$$

Then we obtain an inclusion

$$
R(-1) \stackrel{c}{\rightarrow} R
$$

with cokernel contained in $\bigoplus_{n} \operatorname{Ext}^{1}\left(s^{n}(F / s F), F\right)$. Now by Serre duality we have $\operatorname{dim} \operatorname{Ext}^{1}\left(s^{n}(F / s F), F\right)=\operatorname{dim} \operatorname{Hom}\left(\tau^{-1} F, s^{n}(F / s F)\right)$. Since by (IV.3.5), $F / s F=$ $\bigoplus_{i \in J} S_{i} \otimes \operatorname{Hom}\left(F, S_{i}\right)^{*}$ we find that $\operatorname{Hom}\left(\tau^{-1} F, s^{n}(F / s F)\right) \cong \bigoplus_{i \in J} \operatorname{Hom}\left(F, S_{v^{n+1}(i)}\right)$ $\otimes \operatorname{Hom}\left(F, S_{i}\right)^{*}$. Thus the dimension of $\operatorname{Hom}\left(\tau^{-1} F, s^{n}(F / s F)\right)$ is bounded independently of $n$ (using the fact that $s$ has finite order).

Step 4. $R$ is commutative. To see this we compute $D=\operatorname{Hom}_{\mathcal{C} / \mathcal{T}}(\pi F, \pi F)$ using the equivalence $\operatorname{qgr}(R) \sim \mathcal{C}$. We denote the quotient map $\operatorname{gr}(R) \rightarrow \operatorname{qgr}(R)$ also by $\pi$. We find

$$
\begin{aligned}
& \operatorname{Hom}_{\mathcal{C} / \mathcal{T}}(\pi F, \pi F)=\lim _{\substack{0 \neq G \subset F \\
\longrightarrow}} \operatorname{Hom}_{\mathcal{C}}(G, F) \\
& =\underset{0 \neq I \subset R}{\lim _{0 \neq I}} \operatorname{Hom}_{\mathrm{qgr}(R)}(\pi I, \pi R) \\
& =\underset{0 \neq J \subset R}{\lim _{0 \neq}} \operatorname{Hom}_{\operatorname{gr}(R)}(J, R) .
\end{aligned}
$$

Hence it follows that $D$ is equal to the degree zero part of the graded quotient field of $R$. Since $R$ has GK-dimension 2, it follows from [1] that $D$ is commutative. Since $R$ is included in $D\left[c, c^{-1}\right]$, it follows that $R$ is also commutative.

Using Proposition $\amalg .2 .3$ together with the fact that $\mathcal{C}$ has no non-zero projective objects, we have now proved the following.

Theorem IV.4.2. Assume that $\mathcal{C}$ is a connected Ext-finite noetherian hereditary abelian category with Serre functor and no non-zero projective objects, and assume that the $\tau$-orbits of the simple objects are finite. Then $\mathcal{C}$ is equivalent to the category 
coh $\mathcal{O}$ of coherent sheaves over a sheaf $\mathcal{O}$ of hereditary orders over a non-singular connected projective curve.

This theorem is sufficient for our classification result. However with only a little more work one can prove the following refinement. We leave the proof to the reader.

Theorem IV.4.3. The following are equivalent:

(a) $\mathcal{C}$ is a connected Ext-finite noetherian hereditary abelian category with Serre functor and no non-zero projective objects, and the orbits of the simple objects are finite.

(b) $\mathcal{C}$ is equivalent to $\operatorname{qgr}(R)$ where $R=k+R_{1}+R_{2}+\cdots$ is a finitely generated commutative domain of Krull dimension two, $R_{1} \neq 0$ and $R$ is an isolated singularity.

(c) $\mathcal{C}$ is equivalent to the category coh $\mathcal{O}$ of coherent sheaves over a sheaf $\mathcal{O}$ of hereditary orders over a non-singular connected projective curve.

IV.5. The infinite orbit case. Let $\mathcal{C}$ satisfy the usual hypotheses and assume in addition that $\mathcal{C}$ is connected and that all $v$-orbits in $I$ are infinite. We show that there are at most two hereditary abelian categories with these properties and that these categories (if they exist) are distinguished by the property of having one or two $\tau$-orbits of simples. Since the $\mathbb{Z} A_{\infty}^{\infty}$ and $\mathbb{Z} D_{\infty}$ categories are examples of hereditary abelian categories with respectively two and one orbits of simples, the proof of the main result in this section (Theorem IV.5.2) is then complete.

We refer to RemarkIV.5.1 below for other approaches towards finishing the proof of Theorem IV.5.2

Following [30] it will be convenient to use injective resolutions of objects in $\mathcal{C}$. Therefore following [11] we let $\tilde{\mathcal{C}}$ be the closure of $\mathcal{C}$ under direct limits. Then $\tilde{\mathcal{C}}$ is also hereditary according to Appendix A.

As before we will separate our analysis into two cases.

\section{Case I. One simple object modulo $\mathcal{T}$.}

Let $F \in \mathcal{F}$ be simple modulo $\mathcal{T}$. Since $\mathcal{C} / \mathcal{T}$ is semisimple and $\tilde{\mathcal{C}}$ is hereditary, the minimal injective resolution of $F$ has the form

$$
0 \rightarrow F \rightarrow E_{1} \rightarrow E_{2} \rightarrow 0
$$

where $E_{1}$ is the injective hull of $F$ and $E_{2} \in \tilde{\mathcal{T}}$.

If $E\left(S_{i}\right)$ denotes the injective hull of $S_{i}$, then $E_{2}=\bigoplus_{i \in I} E\left(S_{i}\right)^{a_{i}}$, where $a_{i}=$ $\operatorname{dim} \operatorname{Ext}^{1}\left(S_{i}, F\right)$.

Now choose $0 \neq T \hookrightarrow E_{2}$ in such way that $T \in \mathcal{T}$. Let $G$ be the inverse image of $T$ in $E_{1}$. Clearly $G \in \mathcal{F}$. Applying $\operatorname{Hom}(G,-)$ to (IV.5.1) we find the exact sequence

$$
0 \rightarrow \operatorname{Hom}(G, F) \rightarrow \operatorname{Hom}\left(G, E_{1}\right) \rightarrow \operatorname{Hom}\left(G, E_{2}\right) \rightarrow \operatorname{Ext}^{1}(G, F) \rightarrow 0 .
$$

Since $F$ and $G$ are simple modulo $\mathcal{T}$ and there exists a non-isomorphism $F \rightarrow G$ (the inclusion), it follows from Lemma IV.1.5 that $\operatorname{Hom}(G, F)=0$. Furthermore by Corollary IV.2.6 we also have $\operatorname{Hom}\left(G, E_{1}\right)=k$. Finally by Serre duality we have $\operatorname{dim} \operatorname{Ext}^{1}(G, F)=\operatorname{dim} \operatorname{Hom}\left(\tau^{-1} F, G\right)$.

Now since $\tau^{-1} F=F$ modulo $\mathcal{T}$, it follows that $E_{1}$ is also the injective hull of $\tau^{-1} F$. Thus there is an inclusion $\tau^{-1} F \rightarrow E_{1}$. By choosing $T$, and hence $G$, large enough we may assume $\tau^{-1} F \subset G$, and hence by Corollary IV.2.6 we have $\operatorname{Hom}\left(\tau^{-1} F, G\right) \cong k$. 
Thus for $G$ large we must have

(IV.5.2)

$\operatorname{dim} \operatorname{Hom}\left(T, E_{2}\right) \leq \operatorname{dim} \operatorname{Hom}\left(G, E_{2}\right)=\operatorname{dim} \operatorname{Hom}\left(G, E_{1}\right)+\operatorname{dim}_{\operatorname{Ext}^{1}}(G, F)=2$.

If $o$ is a $v$-orbit in $I$, then let $b_{o}=\sum_{i \in o} a_{i}$. Note that by Lemma IV.2.2 and Serre duality it follows that $b_{o}$ is finite.

From the description of the category $\mathcal{T}_{o}$ as f.l.gr $(k[x])$ we obtain that we can make $\operatorname{dim} \operatorname{Hom}\left(T, E_{2}\right)$ equal to $\sum_{o \in S} b_{o}^{2}$ for any finite subset $S$ of orbits in $I$. In particular there are at most two orbits for which $b_{o} \neq 0$ and for these orbits $b_{o}=1$. Said differently, $F$ has at most two simple quotients (using Serre duality), and if there are two, then they must lie in distinct $\tau$-orbits.

Assume now that $F$ has exactly one simple quotient $S_{i}$. Applying $\operatorname{Hom}\left(-, E_{2}\right)$ to the exact sequence

$$
0 \rightarrow F \rightarrow G \rightarrow T \rightarrow 0
$$

we find the exact sequence

$$
0 \rightarrow \operatorname{Hom}\left(T, E_{2}\right) \rightarrow \operatorname{Hom}\left(G, E_{2}\right) \rightarrow \operatorname{Hom}\left(F, E_{2}\right) \rightarrow 0 .
$$

By Serre duality $F$ has a unique non-trivial extension by the simple $S_{v^{-1} i}$. By the structure of $\mathcal{T}$ it follows that $E_{2}$ is uniserial with subquotients $S_{v^{-n}}$ for $n>$ 0 , whence $\operatorname{Hom}\left(F, E_{2}\right)=0$. But again using the structure of $\mathcal{T}$ we also have $\operatorname{Hom}\left(T, E_{2}\right) \cong k$. Thus $\operatorname{Hom}\left(G, E_{2}\right) \cong k$, contradicting IV.5.2.

So $F$ has two simple quotients which lie in different $\tau$-orbits. Translating (IV.3.4) to our situation using the description of $\operatorname{pc}\left(C_{o}\right)=\operatorname{gr}(k[x])$ and $\operatorname{qpc}\left(C_{o}\right)=\bmod (k)$ we find that $\mathcal{C}$ is the pullback of

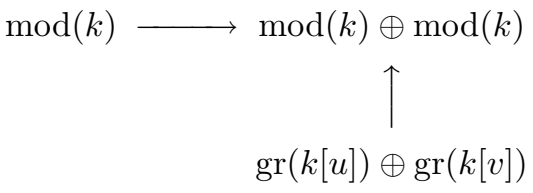

where the horizontal map is the diagonal map and the vertical map is obtained from localizing at $u$ and $v$ and restricting to degree zero. Hence it follows that $\mathcal{C}$ is indeed determined up to equivalence.

\section{Case II. Two simple objects modulo $\mathcal{T}$.}

Let $F_{1}, F_{2} \in \mathcal{F}$ be the representatives of the simple objects in $\mathcal{C} / \mathcal{T}$. As in Case I we start with the minimal injective resolution of $F_{1}$ :

$$
0 \rightarrow F_{1} \rightarrow E_{1} \rightarrow E_{2} \rightarrow 0
$$

With similar reasoning as in Case I, but now using that by Serre duality $\operatorname{Ext}^{1}\left(G, F_{1}\right)$ $=0$ for $G$ torsion free and isomorphic to $F_{1}$ modulo $\mathcal{T}$, we find that $F_{1}$ has a unique simple quotient. The same holds for $F_{2}$, and by Corollary IV.1.8 these simple quotients must be in the same $\tau$-orbit.

Now translating (IV.3.4 to our situation we find that $\mathcal{C}$ is the pullback of

$$
\begin{gathered}
\bmod (k) \oplus \bmod (k) \longrightarrow \\
\underset{\operatorname{gr}(k[x])}{\bmod (k)}
\end{gathered}
$$

where the horizontal map sends $\left(V_{1}, V_{2}\right)$ to $V_{1} \oplus V_{2}$ and the vertical map is localizing at $x$ and restricting to degree zero. Thus $\mathcal{C}$ is again uniquely determined. 
By the argument given in the beginning of this section the proof of the main result is now complete.

Remark IV.5.1. In this remark we indicate some alternative arguments that could have been used in this section.

1. Consider the case where there is one simple object modulo $\mathcal{T}$. In that case we could have observed (once we have proved that there are exactly two $\tau$-orbits of simples) that the functor $s$ defined in $\$ \mathrm{IV} .3$ defines a "hypersurface" in $\mathcal{C}$ in the sense of [30]. Then it follows automatically from the results in [30] that $\mathcal{C}$ is determined up to equivalence and is given by the category which was denoted by $\mathcal{C}$ in $\amalg I .3 .7$ Unfortunately there is no corresponding approach for the case where there are two simple objects modulo $\mathcal{T}$.

2. Another approach is that instead of constructing the $\mathbb{Z} A_{\infty}^{\infty}$ and $\mathbb{Z} D_{\infty}$ categories beforehand we may actually show that the categories defined by the pullback diagrams (IV.5.3) and (IV.5.4) have the expected properties (hereditary abelian, existence of almost split sequences, etc.). This is fairly easy for the diagram (IV.5.3) since one easily shows that in that case the resulting category is equivalent to the one which was denoted by $\mathcal{C}$ in 11 II.3.7 (invoking the results in [30, or directly). If the characteristic is different from two, then diagram (IV.5.4) is obtained by a skew group construction from diagram (IV.5.3), so this case is easy too by the results in [22].

Unfortunately if one wants to include the characteristic 2 case there is no alternative but to give a direct proof for diagram (IV.5.4). This can indeed be done, but requires some work.

We now summarize the main result in this section.

Theorem IV.5.2. The connected hereditary abelian noetherian Ext-finite categories with Serre functor and some object of infinite length and no non-zero projective object are exactly those of one of the following forms:

1. The category of coherent sheaves over a hereditary order over a connected non-singular projective curve.

2. The $\mathbb{Z} A_{\infty}$ category.

3. The $\mathbb{Z} D_{\infty}$ category.

Combining Theorem IV.5.2 with Theorem III.1.1 and Theorem II.4.9 gives the proof of Theorem B.

\section{Applications}

In this section we give some application of our classification theorem to saturated categories and to graded rings.

An Ext-finite abelian category of finite homological dimension is said to be saturated if every cohomological functor of finite type $D^{b}(\mathcal{C}) \rightarrow \bmod (k)$ is representable [8]. We will use our classification result to show that connected saturated noetherian hereditary abelian categories are either equivalent to $\bmod \Lambda$ for some indecomposable finite dimensional hereditary $k$-algebra $\Lambda$ or to the category $\operatorname{coh} \mathcal{O}$ for a sheaf of hereditary orders over a non-singular connected projective curve. This result will also apply to some categories of the form $\operatorname{qgr}(R)$ since these are often saturated. 
V.1. Saturatedness. In this section we give the application to saturated hereditary abelian categories.

Let $\mathcal{A}$ be a Hom-finite triangulated category of finite homological dimension. Following Bondal and Kapranov [8] we say that $\mathcal{A}$ is saturated if every cohomological functor of finite type $H: \mathcal{A} \rightarrow \bmod (k)$ is representable. Finite type means that for every $A \in \mathcal{A}$ at most a finite number of $H(A[n])$ are non-zero.

If $\mathcal{C}$ is an Ext-finite abelian category of finite homological dimension, then we say that $\mathcal{C}$ is saturated if the same holds for $D^{b}(\mathcal{C})$.

In $[8]$ it is shown that the following two categories are saturated:

1. $\bmod (\Lambda)$ where $\Lambda$ is a finite dimensional algebra of finite global dimension.

2. $\operatorname{coh}(X)$ where $X$ is a non-singular projective variety over $k$.

We start with the following easy lemma which is a kind of converse to 1 .

Lemma V.1.1. Assume that $\mathcal{C}$ is an Ext-finite abelian category of finite homological dimension in which every object has finite length. Then $\mathcal{C}$ is saturated if and only if $\mathcal{C} \cong \bmod (\Lambda)$ for $\Lambda$ a finite dimensional algebra of finite global dimension.

Proof. That $\bmod (\Lambda)$ has the desired properties has already been stated, so we prove the converse.

We embed $\mathcal{C}$ into its closure under direct limits $\tilde{\mathcal{C}}$ (see [11]). Let $E$ be the direct sum of the injective hulls of the simples in $\mathcal{C}$. If $S \in \mathcal{C}$ is simple, then clearly $\operatorname{Hom}(S, E) \cong k$ and hence

$$
\operatorname{dim} \operatorname{Hom}(M, E)=\operatorname{length} M
$$

for $M$ in $\mathcal{C}$. For use below we note that it is easy to see that (V.1.1) holds even for $M \in \tilde{\mathcal{C}}$

It follows from (V.1.1) that $F=\operatorname{Hom}(-, E)^{*}$ defines a cohomological functor $D^{b}(\mathcal{C}) \rightarrow \bmod (k)$. Since $\mathcal{C}$ is saturated, this functor is representable by some object $P \in D^{b}(\mathcal{C})$, and since the restriction of $F$ is zero on $\mathcal{C}[n]$ for $n \neq 0$ and exact on $\mathcal{C}$, it follows that $P$ is actually a projective object in $\mathcal{C}$.

Hence we now have $\operatorname{Hom}(P,-)^{*} \cong \operatorname{Hom}(-, E)$ as functors on $\mathcal{C}$. Applying proj $\lim$ it follows that this also holds as functors on $\tilde{\mathcal{C}}$. Thus we have $\operatorname{Hom}(P, E) \cong$ $\operatorname{Hom}(E, E)^{*}$. Using (V.1.1) we find that length $E=$ length $P<\infty$. Thus $E$ is an injective cogenerator of $\mathcal{C}$ and hence a projective generator of $\mathcal{C}^{\text {opp }}$. It follows that $\mathcal{C}^{\text {opp }}$ is equivalent to $\bmod \left(\Lambda^{\text {opp }}\right)$ where $\Lambda=\operatorname{End}(E)$. Since $\mathcal{C}^{\text {opp }}$ has finite homological dimension, so does $\Lambda$. Furthermore

$$
\mathcal{C}=\bmod \left(\Lambda^{\mathrm{opp}}\right)^{\mathrm{opp}}=\bmod (\Lambda)
$$

(using the functor $(-)^{*}$ ). This finishes the proof.

We now have the following result for hereditary abelian categories. Note that it is not hard to see that if $\mathcal{C}$ is saturated, then $\mathcal{C}$ has Serre duality.

Theorem V.1.2. Assume that $\mathcal{C}$ is a connected noetherian Ext-finite hereditary abelian category. Then $\mathcal{C}$ is saturated if and only if it has one of the following forms:

1. $\bmod (\Lambda)$ where $\Lambda$ is a connected finite dimensional hereditary algebra.

2. $\operatorname{coh}(\mathcal{O})$ where $\mathcal{O}$ is a sheaf of hereditary $\mathcal{O}_{X}$-orders (see III.2) over a nonsingular connected projective curve $X$. 
Proof. We already know that the category in 1 . has the required properties. That this is so for the category in 2 . follows in the same way as for $\operatorname{coh}(X)$ where $X$ is a non-singular proper curve.

We now prove the converse. Assume that $\mathcal{C}$ is not of the form 2. This means by Theorem $\mathrm{B}$ that it has one of the following forms:

(a) $\widetilde{\operatorname{rep}}(Q)$ for $Q$ a star.

(b) The $\mathbb{Z} A_{\infty}^{\infty}$ or the $\mathbb{Z} D_{\infty}$ category.

(c) Finite dimensional nilpotent representations over $\tilde{A}_{n}$ or $A_{\infty}^{\infty}$, with all arrows oriented in the same direction.

In the categories in (c) all objects are of finite length but they are clearly not of the form $\bmod (\Lambda)$ since they have no projectives. Hence by Lemma $\nabla .1 .1$ it follows that $\mathcal{C}$ is not of type $(\mathrm{c})$.

By construction the categories in (b) are derived equivalent to the finite dimensional representations over an infinite quiver. Hence again by Lemma V.1.1 the categories in (b) are not saturated.

So we are left with (a). Since $Q$ is a star, there is clearly a section $Q^{\prime}$ in $\mathbb{Z} Q$ such that all paths in $Q^{\prime}$ are finite. Then the indecomposable projective and injective representations of $Q^{\prime}$ have finite length. By Theorem $\llbracket$ II.3.6 we know that $\widetilde{\mathrm{rep}}(Q)$ is derived equivalent to $\operatorname{rep}\left(Q^{\prime}\right)$, and all objects in $\operatorname{rep}\left(Q^{\prime}\right)$ have finite length. Again by Lemma V.1.1 we know that $\operatorname{rep}\left(Q^{\prime}\right)$ is saturated if and only if $Q^{\prime}$ is finite. But then the same holds for $\widetilde{\operatorname{rep}}(Q)$. We conclude that $\mathcal{C}$ is of the form $\widetilde{\operatorname{rep}}(Q)=\operatorname{rep}(Q)$ with $Q$ finite.

Remark V.1.3. Abelian categories satisfying the hypotheses of the previous theorem (except noetherianness) can, in Bondal's terminology [10, be viewed as "noncommutative non-singular proper curves". It appears that non-commutative curves are actually quite close to commutative ones. Other manifestations of this principle are [28, 1, 2].

V.2. Graded rings. In this section we give the application to the hereditary abelian categories of the form $\operatorname{qgr}(R)$. We start with recalling the definition of the condition $\chi$ from [3]. Let $R=k+R_{1}+R_{2}+\cdots$ be an $\mathbb{N}$-graded ring which is right noetherian. Let $m=R_{1}+R_{2}+\cdots$ be the graded maximal ideal of $R$. For $M$ in $\operatorname{Gr}(R)$ we denote by $\Gamma_{m}(M)$ the maximal torsion submodule of $M$, that is, the maximal submodule of $M$ which is locally annihilated by $m$. Then $R$ is said to satisfy condition $\chi$ if for all $M$ in $\operatorname{gr}(R)$ and all $i$ we have that the right derived functor $R^{i} \Gamma_{m}(M)$ has right bounded grading.

The following is a special case of a result proved in [9].

Theorem V.2.1 (9]). Let $R=k \oplus R_{1} \oplus R_{2} \oplus \cdots$ be a right noetherian graded ring satisfying the following hypotheses:

1. $R$ satisfies $\chi$.

2. $\Gamma_{m}$ has finite cohomological dimension and the same condition on the left.

3. $\operatorname{Ext}_{R}^{n}\left(k,{ }_{R} R\right.$ ) is finite dimensional for all $n$ (this is a special case of the $\chi$ condition for left modules).

4. There is an $m \in \mathbb{N}$ such that $\operatorname{Ext}_{\mathrm{qgr}(R)}^{n}(A, B)=0$ for all $A, B \in \operatorname{qgr}(R)$ and $n>m$.

Then $\operatorname{qgr}(R)$ is saturated.

From this result we obtain the following consequence. 
Corollary V.2.2. Assume that $R$ satisfies the hypotheses of Theorem V.2.1 and that $\operatorname{qgr}(R)$ is in addition hereditary. Then $\operatorname{qgr}(R)$ is a direct sum of categories of the following form:

1. $\bmod (\Lambda)$ where $\Lambda$ is a connected finite dimensional hereditary algebra.

2. $\operatorname{coh}(\mathcal{O})$ where $\mathcal{O}$ is a sheaf of hereditary $\mathcal{O}_{X}$-orders (see $\left.§ \amalg I .2\right)$ over a nonsingular connected projective curve $X$.

\section{Appendix A. Some Results on abelian Categories}

Here we give a few homological results on abelian categories that have been used before. Since they have a somewhat different flavor from the other results in this paper we have decided to collect them here in an appendix.

The first result (Proposition A.2 below) implies in particular that if we take all finite length objects in a hereditary abelian category we get again a hereditary abelian category. The present proof of Proposition A.2 is based on the following lemma, where the proof is included for the convenience of the reader.

Lemma A.1. Assume that $\mathcal{H}$ is an abelian category. Then $\mathcal{H}$ is hereditary abelian if and only if $\operatorname{Ext}_{\mathcal{H}}^{1}(X,-)$ preserves epis for all $X \in \mathcal{H}$.

Proof. Let us prove the non-obvious implication. Thus assume that $\operatorname{Ext}_{\mathcal{H}}^{1}(X,-)$ preserves epis. Let

$$
0 \rightarrow A \stackrel{u}{\longrightarrow} B \stackrel{v}{\rightarrow} C \stackrel{w}{\longrightarrow} D \rightarrow 0
$$

be an exact sequence. We have to show that it is Yoneda equivalent to a split one.

Let $K=\operatorname{ker} w$. Then we have a surjective map $B \rightarrow K$, as well as an exact sequence $0 \rightarrow K \rightarrow C \rightarrow D \rightarrow 0$, representing an element of $\operatorname{Ext}_{\mathcal{H}}^{1}(D, K)$. By the fact that $\operatorname{Ext}_{\mathcal{H}}^{1}(D,-)$ preserves epis, this can be lifted to an element of $\operatorname{Ext}_{\mathcal{H}}^{1}(D, B)$. Thus we obtain a commutative diagram with exact rows:

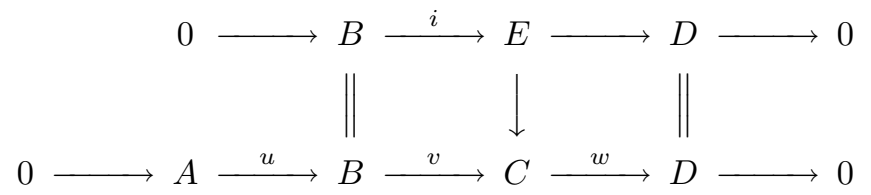

which can be transformed into the diagram

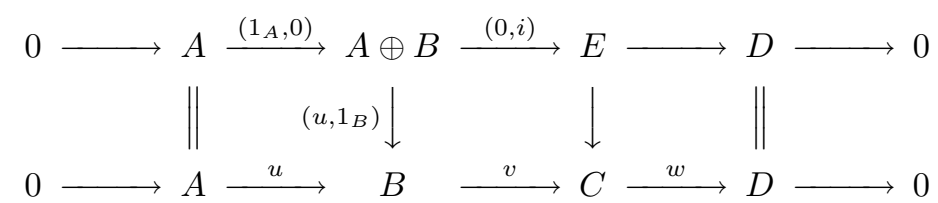

from which it follows that (A.1) represents a trivial class in $\operatorname{Ext}_{\mathcal{H}}^{2}(D, A)$.

Proposition A.2. Let $\mathcal{H}$ be a hereditary abelian category. Then any Serre subcategory $\mathcal{H}^{\prime}$ of $\mathcal{H}$ is hereditary.

Proof. This now follows from the previous lemma using the fact that for $X, Y \in \mathcal{H}^{\prime}$ one has $\operatorname{Ext}_{\mathcal{H}^{\prime}}^{1}(X, Y) \cong \operatorname{Ext}_{\mathcal{H}}^{1}(X, Y)$ (since $\mathcal{H}^{\prime}$ is closed under extensions).

If one has a noetherian abelian category $\mathcal{H}$ of finite homological dimension, then one sometimes needs to go to the closure $\tilde{\mathcal{H}}$ of $\mathcal{H}$ under direct limits. It follows from the following proposition that this operation preserves homological dimension. 
Proposition A.3. Let $\mathcal{X}$ be a noetherian abelian category where the isomorphism classes of objects form a set, and assume $\mathcal{X} \subset \tilde{\mathcal{X}}$ where $\tilde{\mathcal{X}}$ is an abelian category where $\tilde{\mathcal{X}}$ is the completion of $\mathcal{X}$ with respect to direct limits of objects in $\mathcal{X}$ (see [11]). Then gl. $\operatorname{dim} \mathcal{X}=$ gl. $\operatorname{dim} \tilde{\mathcal{X}}$.

Proof. (i) We first show gl. $\operatorname{dim} \mathcal{X} \leq$ gl. $\operatorname{dim} \tilde{\mathcal{X}}$. We have that $\mathcal{X}$ is a thick (Serre) abelian subcategory of $\tilde{\mathcal{X}}$. Let $f: B \rightarrow C$ be an epimorphism in $\tilde{X}$, with $C$ in $\mathcal{X}$. We have by assumption $B=\lim _{i} B_{i}$, where each $B_{i}$ is in $\mathcal{X}$. Since $C$ is noetherian, there is some $j$ such that the induced map $B_{j} \rightarrow C$ is an epimorphism. By the dual of [18, 1.7.11] we have an isomorphism of derived categories $D^{b}(\mathcal{X}) \simeq D_{\mathcal{X}}^{b}(\tilde{\mathcal{X}})$, and in particular $\operatorname{Ext}_{\mathcal{X}}^{t}(X, Y) \simeq \operatorname{Ext}_{\tilde{\mathcal{X}}}^{t}(X, Y)$ for $X$ and $Y$ in $\mathcal{X}$. It follows that gl. $\operatorname{dim} \mathcal{X} \leq$ gl. $\operatorname{dim} \tilde{\mathcal{X}}$.

(ii) Assume now that gl. $\operatorname{dim} \mathcal{X}=r$. We want to show that for $M$ and $N$ in $\tilde{\mathcal{X}}$ we have $\operatorname{Ext}_{\tilde{\mathcal{X}}}^{r+1}(M, N)=0$. If $M$ and $N$ are both in $\mathcal{X}$, this follows from the above, since $\operatorname{Ext}_{\tilde{\mathcal{X}}}^{r+1}(M, N) \simeq \operatorname{Ext}_{\mathcal{X}}^{r+1}(M, N)$, which is 0 by assumption. If $M$ is in $\mathcal{X}$, and hence by assumption is noetherian, $\operatorname{Hom}_{\tilde{\mathcal{X}}}(M$,$) commutes with \underline{\lim }_{i}$, and hence the same is true for the derived functors [13]. It then follows that $\operatorname{Ext}_{\mathcal{X}}^{r+1}(M, N)=0$ if $M$ is in $\mathcal{X}$.

Now let $N$ be in $\tilde{\mathcal{X}}$, and consider the exact sequence

$$
0 \rightarrow N \rightarrow E_{0} \rightarrow E_{1} \rightarrow \cdots \rightarrow E_{r-1} \stackrel{f_{r-1}}{\longrightarrow} E_{r} \stackrel{f_{r}}{\longrightarrow} Z_{r+1} \rightarrow 0,
$$

where the $E_{i}$ are injective. Then we have

$$
\operatorname{Ext}_{\tilde{\mathcal{X}}}^{r+1}(M, N) \simeq \operatorname{Cok}\left(\operatorname{Hom}_{\tilde{\mathcal{X}}}\left(M, E_{r}\right) \rightarrow \operatorname{Hom}_{\tilde{\mathcal{X}}}\left(M, Z_{r+1}\right)\right)
$$

for all $M$ in $\tilde{\mathcal{X}}$. In particular, for any inclusion map $j: M \rightarrow Z_{r+1}$ with $M$ in $\mathcal{X}$ there is a commutative diagram

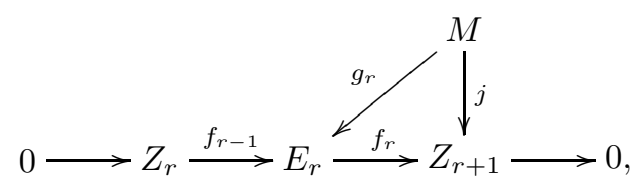

where $Z_{r}=\operatorname{im} f_{r-1}$. If we have a commutative diagram

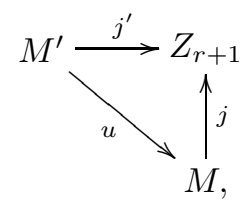

with $M$ and $M^{\prime}$ in $\tilde{\mathcal{X}}$ and $u$ a monomorphism, we then have $g_{r}^{\prime}: M^{\prime} \rightarrow E_{r}$ with $f_{r} g_{r}^{\prime}=j^{\prime}$ and $g_{r}: M \rightarrow E_{r}$ with $f_{r} g_{r}=j$, but we do not necessarily have $g_{r} u=$ $g_{r}^{\prime}$. To adjust the map $g_{r}$ observe that $f_{r}\left(g_{r} u-g_{r}^{\prime}\right)=0$. Hence there is a map $t: M^{\prime} \rightarrow Z_{r}$ with $f_{r-1} t=g_{r} u-g_{r}^{\prime}$. Since $\operatorname{Ext}_{\tilde{\mathcal{X}}}^{1}\left(M / M^{\prime}, Z_{r}\right) \simeq \operatorname{Ext}_{\tilde{\mathcal{X}}}^{r+1}\left(M / M^{\prime}, N\right)=$ 0 because $M / M^{\prime}$ is in $\mathcal{X}$, there is a map $s: M \rightarrow Z_{r}$ such that $s u=t$, and hence $f_{r-1} s u=g_{r} u-g_{r}^{\prime}$. Letting $\overline{g_{r}}=-g_{r}+f_{r-1} s$ we see that $\overline{g_{r}} u=g_{r}^{\prime}$ as desired.

We now use Zorn's lemma to choose a maximal pair $\left(j: T \hookrightarrow Z_{r+1}, \phi: T \rightarrow\right.$ $E_{r}$ with $\left.f_{r} \phi=j\right)$. Assume that $T \neq Z_{r+1}$. Since $Z_{r+1}$ is the direct limit of objects in $\mathcal{X}$, there is some $M \subset Z_{r+1}$ with $M$ in $\mathcal{X}$ and $M \not \subset T$. Consider $M \cap T \subset T \subset Z_{r+1}$ and the map $\left.\phi\right|_{M \cap T}: M \cap T \rightarrow E_{r}$. Then there is by the first 
part of the proof some map $\phi^{\prime}: M \rightarrow E_{r}$ such that $f_{r} \phi^{\prime}=i$ with $i: M \rightarrow Z_{r+1}$, and $\left.\phi^{\prime}\right|_{M \cap T}=\left.\phi\right|_{M \cap T}$. Hence the map $\phi^{\prime}-\phi: M \oplus T \rightarrow E_{r}$ factors through a map $\tilde{\phi}: M+T \rightarrow E_{r}$. This map has the property that $f_{r} \tilde{\phi}=i^{\prime}$ with $i^{\prime}: T+$ $M \rightarrow Z_{r+1}$. This contradicts the maximality of $\left(j: T \hookrightarrow Z_{r+1}, \phi: T \rightarrow E_{r}\right)$, and hence $T=Z_{r+1}$. This means that $f_{r}: E_{r} \rightarrow Z_{r+1}$ is a split epimorphism, and hence $\operatorname{id}_{\tilde{\mathcal{X}}} N \leq r$, so that $\operatorname{Ext}_{\tilde{\mathcal{X}}}^{r+1}(M, N)=0$ for all $M$ in $\tilde{\mathcal{X}}$. Hence we have gl. $\operatorname{dim} \tilde{\mathcal{X}} \leq r=$ gl. $\operatorname{dim} \mathcal{X}$.

Now we consider quotient categories. Recall that a Grothendieck category is an abelian category with a generator and exact direct limits. Such categories automatically have products and injective hulls 31. Prominent examples of Grothendieck categories are locally noetherian categories. These are the abelian categories which are the closure of noetherian categories under direct limits (that is: categories of the form $\tilde{\mathcal{X}}$ as considered in the previous proposition). A localizing subcategory $\mathcal{C}$ of a Grothendieck category $\mathcal{A}$ is a Serre subcategory which is closed under direct limits. In that case $\mathcal{A} / \mathcal{C}$ is also a Grothendieck category. $\mathcal{C}$ is called stable if it is closed under injective hulls in $\mathcal{A}$, or equivalently, if it is closed under essential extensions. The following result is proved in [31] (and is easy to see).

Proposition A.4. Let $\mathcal{A}$ be a Grothendieck category and $\mathcal{C}$ a stable localizing subcategory. Then the quotient functor $T: \mathcal{A} \rightarrow \mathcal{A} / \mathcal{C}$ preserves injective hulls and in particular $\operatorname{gl} \operatorname{dim} \mathcal{A} / \mathcal{C} \leq \operatorname{gl} \operatorname{dim} \mathcal{A}$.

\section{REFERENCES}

[1] M. Artin and J. T. Stafford, Noncommutative graded domains with quadratic growth, Invent. Math. 122 (1995), 231-276. MR 96g:16027

[2] M. Artin and J. T. Stafford, Semiprime graded algebras of dimension two, J. Algebra 227 (2000), no. 1, 68-123. CMP 2000:12

[3] M. Artin and J. J. Zhang, Noncommutative projective schemes, Adv. in Math. 109 (1994), no. 2, 228-287. MR 96a:14004

[4] M. Auslander and I. Reiten, Almost split sequences in dimension two, Adv. in Math. 66 (1987), no. 1, 88-118. MR 89a:16039

[5] M. Auslander, I. Reiten, and S. Smalø, Representation theory of Artin algebras, Cambridge Studies in Advanced Mathematics, vol. 36, Cambridge University Press, 1995. MR 96c:16015

[6] A. Beilinson, On the derived category of perverse sheaves, K-theory, Arithmetic and Geometry, Lecture Notes in Mathematics, vol. 1289, Springer Verlag, 1987, pp. 27-41. MR 89b:14027

[7] A. Beilinson, J. Bernstein, and P. Deligne, Faisceaux pervers, Astérisque, vol. 100, Soc. Math. France, 1983. MR 86g:32015

[8] A. I. Bondal and M. M. Kapranov, Representable functors, Serre functors, and reconstructions, Izv. Akad. Nauk SSSR Ser. Mat. 53 (1989), no. 6, 1183-1205, 1337. MR 91b:14013

[9] A. I. Bondal and M. Van den Bergh, Generators of triangulated categories and representability of functors, in preparation.

[10] A. I. Bondal, Non-commutative deformations and Poisson brackets on projective spaces, MPI-preprint, 1993.

[11] P. Gabriel, Des catégories abéliennes, Bull. Soc. Math. France 90 (1962), 323-448. MR 38:1144

[12] W. Geigle and H. Lenzing, A class of weighted projective curves arising in representation theory of finite dimensional algebras, in: Singularities, representations of algebras and vector bundles, Lecture Notes in Mathematics, vol. 1274, Springer Verlag, 1987, pp. 265-297. MR 89b:14049

[13] A. Grothendieck, Sur quelques points d'algèbre homologiques, Tôhoku Math. J. (2) 9 (1957), 119-221. MR 21:1328 
[14] D. Happel, Triangulated categories in the representation theory of finite dimensional algebras, London Mathematical Society lecture note series, vol. 119, Cambridge University Press, 1988. MR 89e:16035

[15] D. Happel, A characterization of hereditary categories with tilting object, Invent. Math. 144 (2001), no. 2, 381-398. MR 2002a:18014

[16] D. Happel, I. Reiten, and S. Smalø, Tilting in abelian categories and quasitilted algebra, Memoirs of the AMS, vol. 575, Amer. Math. Soc., 1996. MR 97j:16009

[17] R. Hartshorne, Algebraic geometry, Springer Verlag, 1977. MR 57:3116

[18] M. Kashiwara and P. Schapira, Sheaves on manifolds, Die Grundlehren der mathematischen Wissenschaften, vol. 292, Springer Verlag, 1994. MR 92a:58132

[19] H. Lenzing, Hereditary noetherian categories with a tilting object, Proc. Amer. Math. Soc. 125 (1997), 1893-1901. MR 98c:16013

[20] J. C. McConnell and J. C. Robson, Noncommutative Noetherian rings, John Wiley \& Sons, New York, 1987. MR 89j:16023

[21] I. Reiner, Maximal orders, Academic Press, New York, 1975. MR 52:13910

[22] I. Reiten and C. Riedtmann, Skew group algebras in the representation theory of artin algebras, J. Algebra 92 (1985), 224-282. MR 86k:16024

[23] I. Reiten and M. Van den Bergh, Grothendieck groups and tilting objects, Algebras and Representation Theory 4 (2001), 257-272.

[24] C. M. Ringel, A ray quiver construction of hereditary abelian categories with Serre duality, Proc. ICRA IX, Beijing.

[25] C. M. Ringel, The diamond category of a locally discrete ordered set, Proc. ICRA IX, Beijing.

[26] C. Robson and L. Small, Hereditary prime PI rings are classical hereditary orders, J. London Math. Soc. (2) 8 (1974), 499-503. MR 50:2236

[27] A. Schofield, unpublished.

[28] L. W. Small, J. T. Stafford, and R. B. Warfield, Affine algebras of Gelfand-Kirillov dimension one are PI, Math. Proc. Cambridge Philos. Soc. 97 (1985), 407-414. MR 86g:16025

[29] S. O. Smalø, Almost split sequences in categories of representations of quivers, Proc. Amer. Math. Soc. 129 (2001), no. 3, 695-698. MR 2001f:16034

[30] S. P. Smith and J. J. Zhang, Curves on quasi-schemes, Algebr. Represent. Theory 1 (1998), no. 4, 311-351. MR 2001a:16046

[31] B. Stenström, Rings of quotients, Die Grundlehren der mathematischen Wissenschaften in Einzeldarstellungen, vol. 217, Springer Verlag, Berlin, 1975. MR 52:10782

[32] M. Van den Bergh, Blowing up of non-commutative smooth surfaces, Memoirs of the AMS, vol. 734, Amer. Math. Soc., 2001.

[33] M. Van den Bergh and M. Van Gastel, Graded modules of Gelfand-Kirillov dimension one over three-dimensional Artin-Schelter regular algebras, J. Algebra 196 (1997), 251-282. MR 99c: 16020

[34] J.-L. Verdier, Des catégories dérivées des catégories abéliennes, Astérisque (1996), no. 239, xii+253 pp. (1997), With a preface by Luc Illusie, Edited and with a note by Georges Maltsiniotis. MR 98c: 18007

Department of Mathematical Sciences, Norwegian University of Science and TechNOLOGY, 7491 Trondheim, NorWAY

E-mail address: idunr@math.ntnu.no

Department Wni, Limburgs Universitair Centrum, Universitaire Campus, Building D, 3590 Diepenbeek, Belgium

E-mail address: vdbergh@luc.ac.be 